University of Louisville

ThinkIR: The University of Louisville's Institutional Repository

Electronic Theses and Dissertations

$5-2020$

\title{
Exosomes from embryonic stem cells as a prophylactic vaccine against lung cancer.
}

Shuhan Meng

University of Louisville

Follow this and additional works at: https://ir.library.louisville.edu/etd

Part of the Life Sciences Commons

\section{Recommended Citation}

Meng, Shuhan, "Exosomes from embryonic stem cells as a prophylactic vaccine against lung cancer." (2020). Electronic Theses and Dissertations. Paper 3373.

https://doi.org/10.18297/etd/3373

This Doctoral Dissertation is brought to you for free and open access by ThinkIR: The University of Louisville's Institutional Repository. It has been accepted for inclusion in Electronic Theses and Dissertations by an authorized administrator of ThinkIR: The University of Louisville's Institutional Repository. This title appears here courtesy of the author, who has retained all other copyrights. For more information, please contact thinkir@louisville.edu. 
EXOSOMES FROM EMBRYONIC STEM CELLS AS A PROPHYLACTIC

VACCINE AGAINST LUNG CANCER

By

\author{
Shuhan Meng \\ B.S Jilin University, 2014 \\ M.D Jilin University, 2016
}

\begin{abstract}
A dissertation
Submitted to the Faculty of the

School of Medicine of the University of Louisville

for the Degree of
\end{abstract}

Doctor of Philosophy in Pharmacology and Toxicology

Department of Pharmacology and Toxicology

University of Louisville

Louisville, KY

May 2020 

EXOSOMES FROM EMBRYONIC STEM CELLS AS A PROPHYLACTIC VACCINE AGAINST LUNG CANCER

\author{
By
}

Shuhan Meng

Dissertation Approved on

April 20, 2020

By the following Dissertation Committee:

\begin{tabular}{c}
\hline Dr. Chi Li \\
\hline Dr. Geoffrey Clark \\
\hline Dr. Joshua Hood \\
\hline Dr. Robert Mitchell
\end{tabular}

Dr. Robert Mitchell

Dr. Yaddanapudi Kavitha 


\section{ACKNOWLEDGEMENTS}

The entire PhD study is a challenge and amazing journey. This is a special time to graduate because of the coronavirus outbreak. I would never finish it without the support of a number of people. Firstly, I am deeply appreciative of my mentor - Dr. Chi Li - for his guidance and support during the entire period of study and research. I still remember the first day when I stepped into his office, his kindness made me feel warm and relieved my anxiety as a foreign student coming to a new country for the first time. I also thank him for his first requirement for me, that we would never speak our mother tongue at school, which made my spoken English improve very fast.

I would like to express my gratitude to my dissertation committee members, Dr. Kavitha Yaddanapudi for her expertise in tumor immunology, Dr. Joshua Hood for his expertise in exosomes, and Dr. Geoffrey Clark and Dr. Robert Mitchell for their expertise in cancer biology. They have provided tremendous help for my graduate research and thesis writing. I would like to thank Dr. John Eaton for his intellectual input, who is a scholar with abundance of knowledge and always has new ideas about research.

I would also like to gratefully thank all the present and former members in our laboratory. They have provided me a lot of kind help and encouragement 
throughout my study. Especially, I want to thank Aaron Whitt, as he has taught me different basic experimental skills and is always willing to answer my questions. Importantly, my appreciation is extended to Dr. Hein and Dr. Cai in our department for their tremendous efforts to build the partnership between the Department of Pharmacology and Toxicology at University of Louisville and Jilin University, providing me an opportunity to study here. I am also grateful to the present and former faculty and staff in our department for their help in conducting research as well as in daily life.

Finally, I would like to extend my deepest and sincerest gratitude to my very supportive family members, especially to my mother - Dr. Xiujuan Zhang - for her selfless love in my life. She told me that she would always have my back and I could just pursue my dreams. Special thanks also go out to my husband Frank Gu for his love and patience. Whenever I feel happy or sad, he is always there. I also want to thank my best friends here, Summer Li and Diane Bramsen, for the valuable friendship between us. I am also grateful to the seniors whom I really respect, Dr. Xiaomei Rao, Dr. Sam Zhou, and Miss Irma for their kind help during the hard and remarkable time. 


\begin{abstract}
EXOSOMES FROM EMBRYONIC STEM CELLS AS A PROPHYLACTIC

VACCINE AGAINST LUNG CANCER
\end{abstract}

Shuhan Meng

April 20, 2020

The antigenic similarity between embryos and tumors has raised the idea of using embryonic materials as a preventative vaccine against neoplastic disease. Indeed, a previous study reported that a vaccine comprised of allogeneic murine embryonic stem cells (ESCs) and murine fibroblasts expressing immune-stimulatory granulocyte macrophage-colony stimulating factor (GMCSF) successfully blocked the outgrowth of an implantable lung cancer (Lewis lung carcinoma; LLC) and lung tumors caused by a combination of a mutagen followed by chronic pulmonary inflammation. However, such a vaccine is obviously impractical for application to humans. The use of fibroblasts to produce GM-CSF is needlessly complicated, and intact live ESCs carry the hazard of generating embryomas/teratomas. 
Here, we report the successful development of an alternative prophylactic vaccine comprised of exosomes/microvesicles derived from murine ESC line ES-D3 engineered to produce GM-CSF. The prophylactic potential of this exosome-based vaccine against implanted lung cancer (subcutaneous inoculation of LLC) was tested by immunizing mice with ESC-derived exosomes bearing GM-CSF (ES-exo/GM-CSF). Vaccination significantly slowed or blocked the outgrowth of LLC without any detectable side effects in liver function, kidney function and blood cell counts, whereas control exosomes lacking GM-CSF were ineffective against LLC. In an implanted lung tumor model, examination of tumor-infiltrating immune cells showed robust tumorreactive immune responses in mice vaccinated with the exosomes bearing GM$\mathrm{CSF}$, including an increase in Th1 cytokine responses, $\mathrm{CD}^{+} \mathrm{T}$ effector responses and $\mathrm{CD} 8^{+} \mathrm{T}$ effector/T regulatory cell ratio.

To further evaluate the efficacy of ES-exo/GM-CSF vaccination in preventing lung tumor development, we investigated an experimental metastasis model where syngeneic LLC cells were administered directly into the bloodstream of wild-type C57BL/6 mice through tail vein injection, primarily resulting in pulmonary metastases. Our studies indicate that vaccination with ES-exo/GM-CSF inhibited metastatic growth of lung tumors. Importantly, control exosomes without GM-CSF failed to provide little protection against metastasized pulmonary malignancies. The efficacy of ES-exo/GM-CSF vaccination was associated with a decrease in tumor-promoting $\mathrm{T}$ regulatory 
cells, myeloid derived suppressor cells (MDSCs) and macrophages in tumor infiltrates as well as an increase in cytokine production from intratumoral CD8 ${ }^{+}$ T cells.

Since the pluripotency is one of the most specialized properties shared between ESCs and a subset of lung tumor cells, cancer stem cells (CSCs), we investigated the role of pluripotency of murine ESCs in prophylactic effectiveness of ESC-based vaccine. The pluripotency of murine ESCs was significantly reduced when they were differentiated into neuronal cells. The pluripotency of ESCs was essential for their anchorage-independent growth potential. Importantly, differentiation decreased the efficacy of ESCs against the outgrowth of implanted lung tumors. Furthermore, long-term cancer-preventive potential of ESC vaccine was also inhibited by differentiation. In summary, these data indicate the importance of pluripotency of ESCs in their prophylactic efficacy against lung cancer.

While ESC-based vaccine has shown great potential, the antigens responsible for its prophylactic efficacy had not been identified yet. Since it is possible that the antibodies generated by ESC vaccine directly bind tumor cells and initiate antibody-dependent cellular cytotoxicity (ADCC), we employed a novel immunoproteomic strategy to gain a deep understanding of antigenicity of ESC-derived vaccine. We examined the comprehensive profiles of antigens specific for the antibodies induced by vaccines based on intact ES-D3 cells or exosomes of ES-D3 cells. Antigens shared by ES-D3 cells and lung tumor cells 
were identified, among which lung tumor-associated keratin members 8, 16, 17 are candidate antigens responsible for initiating anti-lung tumor immunity through ADCC.

Overall, based on the antigenic similarities between embryos and lung tumors, we developed an effective lung cancer vaccine composed of ESCderived exosomes bearing GM-CSF. This vaccine was very effective in preventing both primary and metastasized lung tumors by evoking tumorspecific immunity. Therefore, we conclude that a similar vaccine derived from GM-CSF-expressing human ESCs may be applicable to humans with increased risk of developing lung cancer, such as long-term smoking history, lung cancer family history, and carcinogen exposure.

Key words: Exosomes, embryonic stem cells, immune-stimulatory granulocyte macrophage-colony stimulating factor, prophylactic vaccine, lung cancer 


\section{TABLE OF CONTENTS}

\section{PAGE}

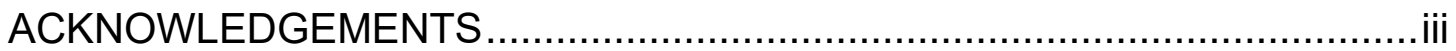

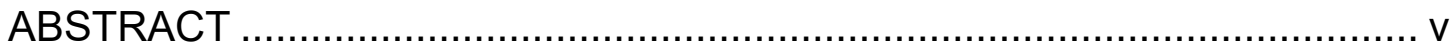

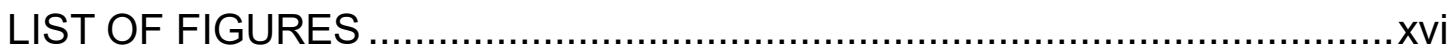

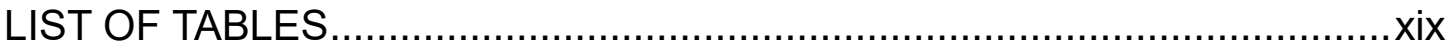

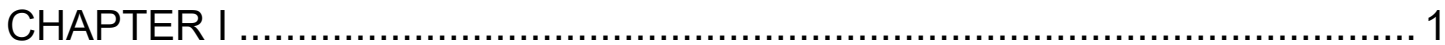

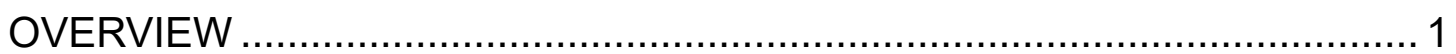

1.1. Lung cancer therapy overview............................................... 1

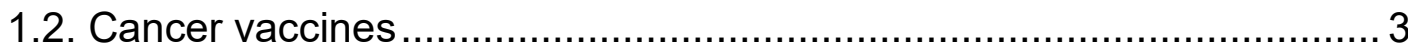

1.2.1. Cancer vaccines classification................................................... 3

1.2.2. Cancer vaccines in development...................................... 6

1.2.3. Cancer vaccine adjuvants ...................................................... 13

1.3. Exosomes in cancer therapys ............................................... 19

1.3.1. Biogenesis of exosomes ...................................................... 19 
1.3.3. Functions of exosomes in tumorigenesis

1.3.4. Exosome-based cancer vaccine

CHAPTER II

EXOSOMES DERIVED FROM MURINE EMBRYONIC STEM CELLS OVEREXPRESSING GM-CSF PREVENT TRANSPLANTED LUNG TUMOR

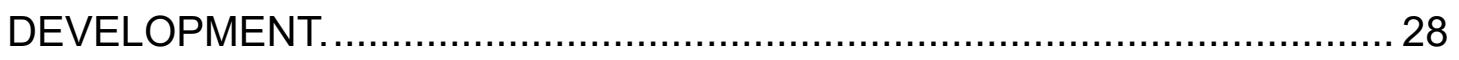

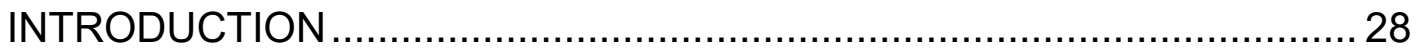

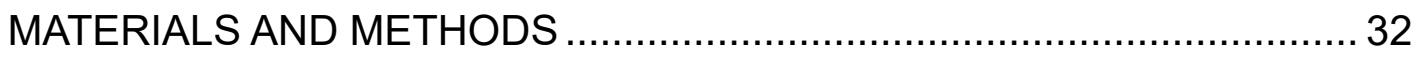

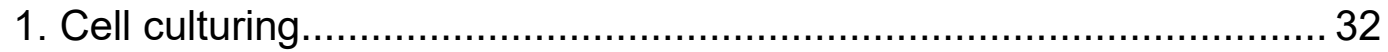

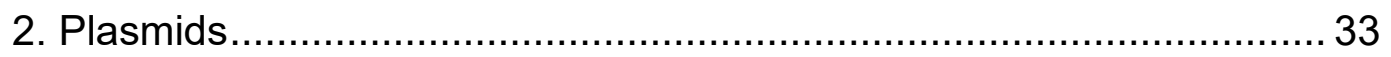

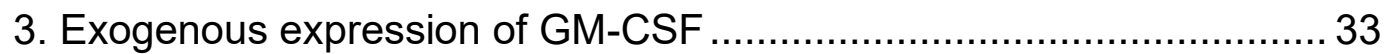

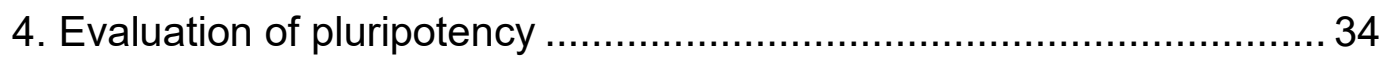

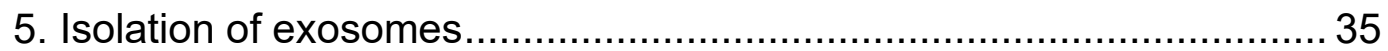

6. Transmission electron microscopy (TEM) …….............................. 35

7. Evaluating exosomes by western blot analysis ................................ 36

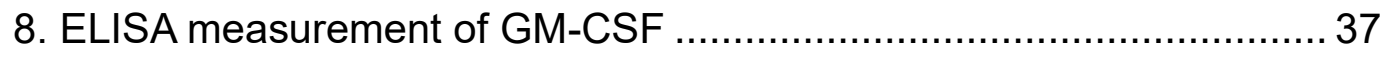


9. Mice.

10. Vaccination and implanted tumor challenge 38

11. Evaluation of Fertility 38

12. Evaluation of toxicological effects. 39

13. Flow cytometric analysis. 39

14. Intracellular cytokine staining 40

15. Analysis of tumor-infiltrating T cells 40

16. Statistical analysis 41

RESULTS 42

1. Exogenous expression of GM-CSF in murine embryonic stem cell ESD3. 42

2. Murine embryonic stem cells expressing GM-CSF maintain their pluripotency. 45

3. Isolation and characterization of exosomes from murine embryonic stem cells. 47

4. The majority of GM-CSF is localized within exosomes. 50

5. Vaccination with GM-CSF-expressing ESC-derived exosomes prevents the outgrowth of implanted lung adenocarcinoma. 52

6. Vaccination with ES-exo/GM-CSF has no toxic side effects in mice. .. 54

7. Vaccination with ES-exo/GM-CSF induces tumor cell-specific Th1- 
mediated cytokine response in $\mathrm{CD} 8^{+} \mathrm{T}$ cells.

8. Vaccination with GMCSF-expressing ESC-derived exosomes increases the ratio of $\mathrm{CD}^{+} \mathrm{T}$ effector cells to $\mathrm{T}_{\text {regs }}$ in the tumor.

9. ESC-derived exosome vaccination prevents the outgrowth of an implanted mammary carcinoma. 66

DISCUSSION 68

CHAPTER III 75

MURINE ESC-DERIVED EXOSOMES FUNCTION AS A CELL-FREE VACCINE AGAINST METASTASIZED LUNG CANCER. 75

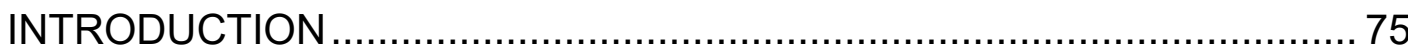

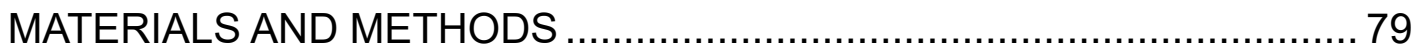

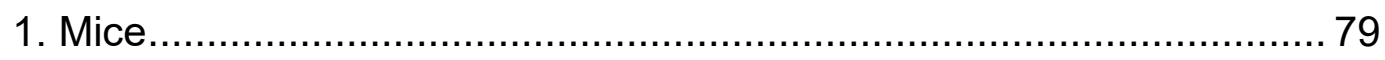

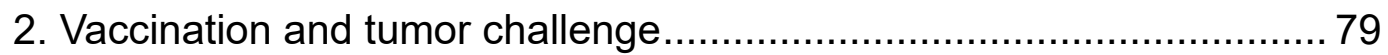

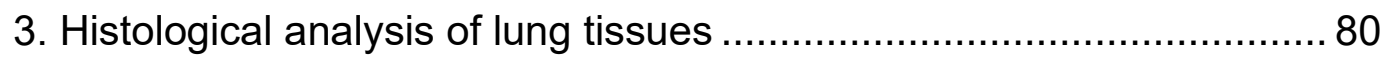

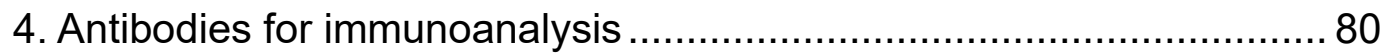

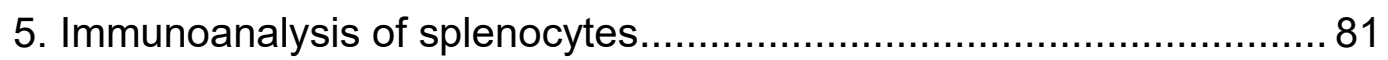

6. Immunoanalysis of tumor-infiltrating immune cells ............................. 81

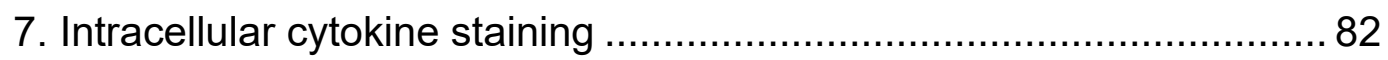

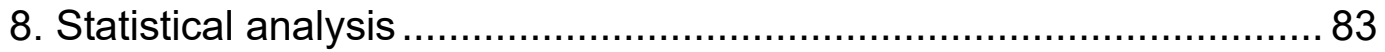


1. Vaccination with ES-exo/GM-CSF inhibit metastasized lung tumor growth

2. ES-exo/GM-CSF vaccination decreases $T$ regulatory cells ( $\left.T_{\text {regs }}\right)$ in lung metastases.

3. Vaccination with ES-exo/GM-CSF suppresses tumor-infiltrating myeloid derived suppressor cells (MDSCs). 92

4. Vaccination with ES-exo/GM-CSF reduces the percentage of macrophages in metastasized lung tumors. 95

5. ES-exo/GM-CSF Vaccination promotes intratumoral B cell populations. 98

6. Intratumoral Th1 and Th17 effector cells are not affected by ES-exo/GMCSF vaccination. 100

7. Vaccination with ES-exo/GM-CSF induces a tumor cell-specific cytokine response in tumor-infiltrating $\mathrm{CD} 8^{+} \mathrm{T}$ cells. 102

DISSCUSION 104

CHAPTER IV 110

THE PLURIPOTENCY OF EMBRYONIC STEM CELLS IS ESSENTIAL FOR CANCER-PREVENTION EFFICACY OF EMBRYONIC STEM CELL-BASED VACCINE. 110 
1. Cell lines

2. Mice

3. Differentiation of murine ESCs

4. Anchorage-independent cell culturing 115

5. Vaccination against tumor challenge 116

6. Proteomics analysis of exosome samples 117

7. Antigen capture experiments for mice immunized with exosomes. ... 118

8. Antigen capture experiments for mice immunized with ESCs. 119

9. Proteomics analysis of protein samples bound to beads 120

10. Statistical analysis

RESULTS 124

1. The pluripotency of murine embryonic stem cells (ESCs) is reduced during differentiation 124

2. The pluripotency of ESCs is important for their anchorage-independent growth potential 128

3. Differentiation decreases the efficacy of ESC vaccine on lung tumor development 130

4. ES-D3 cells and lung tumor cells exhibit antigenic similarity as revealed 
by antigen capture experiments

DISSCUSION

REFERENCE 


\section{LIST OF FIGURES}

Figure 2.1. Strategies to generate vaccines against lung cancer.

Figure 2.2. Exogenous GM-CSF was stably over-expressed in ES-D3 cells. 44

Figure 2.3. Pluripotency of ES-D3 cells is not affected by GM-CSF expression.

Figure 2.4. Characterization of exosomes isolated from ES-D3 cells. 48

Figure 2.5. GM-CSF is located within the exosomes 51

Figure 2.6. Vaccinating mice with exosomes from ESCs expressing GM-CSF prevents implanted lung tumor growth. 53

Figure 2.7. Vaccination with ESC-derived exosomes (ES-exo/GM-CSF) does not reduce fertility of mice 55

Figure 2.8. ES-exo/GM-CSF vaccination does not have display toxic effects on functions of kidney and liver. 56

Figure 2.9. ES-exo/GM-CSF vaccination has no influence on blood cell counts 58

Figure 2.10. ESC-derived exosome vaccination induces Th1-mediated cytokine responses in splenic $\mathrm{CD} 8^{+} \mathrm{T}$ cells. 60 
Figure 2.11. Vaccination with ESC-derived exosomes promotes Th1-mediated cytokine responses in intra-tumoral $\mathrm{CD} 8^{+} \mathrm{T}$ cells. 61

Figure 2.12. ES-exo/GM-CSF vaccination decreases $T$ regulatory ( $T_{\text {regs }}$ ) cells and increases the ratio of effector $\mathrm{CD}^{+} \mathrm{T}$ cells to Tregs in the tumors. 64

Figure 2.13. Vaccination with ES-exo/GM-CSF slows the outgrowth of an implanted mammary carcinoma. 66

Figure 3.1. Vaccination with exosomes isolated from GM-CSF-expressing ESD3 (ES-exo/GM-CSF) cells inhibits the outgrowth of metastasized lung tumors.

Figure 3.2. Vaccination with ES-exo/GM-CSF decreases $T$ regulatory cells (Tregs) in metastasized lung tumors. 90

Figure 3.3. Vaccination with ESCs-exo suppresses tumor-infiltrating MDSCs. 93

Figure 3.4. Vaccination with ES-exo/GM-CSF reduces tumor-infiltrating macrophages. 96

Figure 3.5. ESC-derived exosome vaccination promotes tumor-infiltrating B cells against lung metastases. 99

Figure 3.6. The levels of Th1 and Th17 effector cells in lung metastases are not affected by ES-exo/GM-CSF vaccination. 100

Figure 3.7. Vaccination with ES-exo/GM-CSF promotes cytokine production from CD8+ $T$ cells 102 
Figure 4.1. Differentiated ES-D3 cells are characterized

Figure 4.2. Differentiation inhibits anchorage-independent proliferation of ES-

D3 cells. 129

Figure 4.3. Differentiation decreases the efficacy of ES-D3 cells against lung cancer. 132

Figure 4.4. Differentiation of ES-D3 cells inhibits their ability to prevent lung cancer. 134

Figure 4.5. Differentiation reduces anti-cancer activities of ES-D3 cells...... 135 Figure 4.6. Differentiation decreases long-term cancer prevention potential of ES-D3 cells 137

Figure 4.7. An immunoproteomics strategy to identify the antigens specific for the antibodies generated by vaccines derived from ESCs. 141

Figure 4.8. Summary of identified antigens specific for vaccine-generated antibodies. 143 


\section{LIST OF TABLES}

Table 2.1 Exosome derived from ES-D3 cells express prototype exosomal markers. 49

Table 4.1 Exosomes derived from ES-D3 cells contains various tumor antigens.

Table 4.2. Keratin members recognized by antibodies in the serum of immunized mice. 144

Table 4.3. Keratin members as candidate antigens responsible for the vaccination efficacy of ES-D3- derived vaccines. 145 


\section{CHAPTER I}

\section{OVERVIEW}

\subsection{Lung cancer therapy overview}

Worldwide, lung cancer is the most common cancer among men in terms of both incidence and mortality [1]. Among women, lung cancer has the third highest incidence and is the second after breast cancer in mortality. Current therapeutic strategies for lung cancer include surgery, chemotherapy, radiotherapy, and emerging immunotherapy. However, surgery can be only utilized at the very early stages of lung cancer. Additionally, patients must surpass pulmonary function benchmarks to qualify for surgery. Chemotherapy and radiotherapy usually serve as maintenance treatment after surgery or as first-line therapies for patients who are not eligible for surgery. Unfortunately, non-small cell lung carcinoma (NSCLC), the most prevalent type of lung cancer, is especially insensitive to chemotherapy or radiation therapy [2]. Most patients with NSCLC patients are at advanced stages at the time of diagnosis. As a result, the 5 years survival rate of patients with NSCLC is low [3]. 
Due to the limitation of conventional lung cancer treatments, more and more researchers are focusing on immunotherapy, which promotes the immune system to attack tumor cells either alone or combined with other chemotherapeutics. As a promising therapy to improve patient survival, lung cancer immunotherapy is generally categorized into 5 classes: nonspecific immune stimulation, T cell transfer, immune checkpoint inhibitors, antibodybased therapy and cancer vaccines [4]. These therapeutic strategies for lung cancer are at various phases for clinical applications - some are approved to treat patients, whereas others are still under preclinical studies.

Nonspecific immune stimulation functions by using immunostimulatory agents, such as interferon alpha (INF- $\alpha$ ) and certain interleukins, without targeting cancer cells specifically [4]. $T$ cell transfer therapy is an immunotherapeutic strategy that engineers immune cells of patients to eliminate cancer specifically, including tumor-infiltrating lymphocytes (TIL) therapy and chimeric antigen receptor (CAR) T cell therapy [4]. Immune checkpoint inhibitors are designed to block the "off" signal from immune checkpoints, allowing activated T cells to attack neoplastic cells [5]. Among immune checkpoint inhibitors, antibodies specific for programmed cell death protein 1 (PD-1) (nivolumab and pembrolizumab) and programmed cell death protein ligand 1 (PD-L1) (atezolizumab) have been approved by the US Food and Drug Administration (FDA) to treat patients with NSCLC. Monoclonal antibodies against molecules involved in lung tumorigenesis have been used in 
antibody-based therapy to directly kill lung neoplastic cells or deliver tumorkilling agents to them [5]. The best known example of antibody-based therapy is cetuximab, a monoclonal antibody against the epidermal growth factor receptor (EGFR), which is combined with conventional chemotherapeutic drugs to treat NSCLC patients. Finally, cancer vaccines are a type of immunotherapy to enable the immune system to recognize and destroy lung cancer cells [6]. Despite some progress, it is important to continue developing novel vaccines that boost human immunity to prevent the initiation and progression of lung cancer.

\subsection{Cancer vaccines}

\subsubsection{Cancer vaccine classification}

A cancer vaccine can either prevent tumor development or treat existing malignancy by modulating the immune system [6]. While vaccines designed to prevent cancer initiation and progression are classified as preventive cancer vaccines, vaccines that treat cancer patients are known as therapeutic cancer vaccines.

\subsubsection{Preventive cancer vaccines}

Tremendous success has been achieved in developing prophylactic vaccines against infectious agents. For certain cancer types, the cause of cancer is viral infection, which is relatively simple compared with the complex processes involved in the development of other human cancers and well established [6]. 
While liver cancer has been found to be induced by human hepatitis $B$ virus (HBV), cervical cancer can be caused by human papilloma virus (HPV) infection. Exploiting the rich experience gained against infectious diseases, researchers have developed several preventive cancer vaccines to prevent HBV or HPV infection, resulting in protection against the development of HBV- and HPVcaused cancer. The first preventive cancer vaccine approved by the FDA is an anti-HBV vaccine [7]. Later, two preventive vaccines, Gardasil@ and Cervarix®, gained FDA approval for clinical application for their protection against infection of two HPV subtypes (type 16 and 18) that are responsible for 70 percent of cervical cancer in the world [8].

However, the pathogenesis of most cancer types is very complicated and many factors contribute to the initiation and progression of cancer [6]. Unlike viruses, which are foreign to the human immune system and recognized as "non-self", these cancer cells more closely resemble their healthy, normal counterparts in human body. Thus, tumors bearing "self" antigens are able to evade immune surveillance, making it difficult to develop preventive vaccines. Therefore, more sophisticated strategies are required to generate effective vaccines to prevent cancer that is not associated with infection of a particular virus. It is critical to develop a vaccine that exhibits broader antitumor efficacy to prevent the outgrowth of various cancers.

\subsubsection{Therapeutic cancer vaccines}

Therapeutic cancer vaccines eliminate tumor cells by promoting human 
immune system against cancer [6]. Distinct from preventive cancer vaccines, therapeutic cancer vaccines are designed to target cancer cells directly. Therefore, therapeutic cancer vaccines are used to treat patients with established cancers. Normally, therapeutic cancer vaccines may be generated by patients' own cells, including tumor cells and dendritic cells, which promote robust immune responses against cancer. Sipuleucel-T, a dendritic cell vaccine, is the first immunotherapy product approved by the US FDA to treat metastatic, castration-resistant prostate cancer in men [9]. This vaccine recognizes prostatic acid phosphatase (PAP), an antigen expressed on prostate cancer cells, to promote T-cell-mediated immune responses against prostate cancer cells. Sipuleucel-T is generated by in vitro activation of antigen-presenting cells (APCs) isolated from a patient's blood by an immunostimulatory cytokine and subsequent infusion of activated APCs back into the patient [10]. However, this expensive vaccination strategy only improves overall survival of patients with advanced prostate cancer by about 4 months.

Therapeutic cancer vaccines may also be generated from tumorassociated antigens identified on specific types of cancer cells [6]. Such a vaccine is capable of triggering immune responses in any patient bearing the antigens. Unfortunately, the majority of vaccine-based therapeutic strategies for cancer have been largely unsuccessful [11]. The probability of generating a vaccine with a broad spectrum of anti-cancer activities could be greatly increased when different tumor-associated antigens presented by various 
tumors, but not by adult tissues, are accessible to the immune system. This vaccination strategy is advantageous over other tumor antigen-based treatments, which have shown limited effectiveness in clinical trials, probably due to their inherent shortcomings [12].

\subsubsection{Cancer vaccines in development}

\subsubsection{Embryonic material-based cancer vaccines}

In most tissues, stem cells are the only type of cells capable of self-renewal, whereas other cell types have a much shorter lifespan of days or weeks [13]. Emerging research about cancer immunization focuses on studying cancer cells and stem cells due to the similarities between them. An earlier study has demonstrated that cancer tissue can be viewed as a newborn abnormal organ formed by the accumulation of tumor cells [14]. To eliminate the side effects of vaccination, cancer vaccines should be developed to avoid influencing somatic stem cells.

So far, many of the known cancer cell surface markers can also be detected on adult stem cells [15]. The similarity in cell markers between tumor cells and normal stem cells has provided evidence for researchers to envision that cancers arise from remnant embryonic cells in human body [16]. Except for the same surface markers, cancer cells and embryonic cells also share some common properties, including histological morphology, reduced contact inhibition, high proliferation rate, tissue invasion ability, anaerobic metabolism, 
dedifferentiation status, evasion of immune destruction, secretion of angiogenic factors, and expression of embryonic genes[17].

The history of immunizing animals with fetal tissues to elicit an antitumor response dates back a century [18]. It was found that most types of neoplastic cells express certain embryonal antigens, a phenotype described as 'retrogenetic expression' [19]. Subsequent reports indicate that transplantable and chemically induced tumors could be prevented by vaccination with embryonic materials that induce cancer-specific immunity. The antigens shared between tumors and embryos have been identified during the past century [18] [20]. Initially, the similarities were thought to be restrained within digestive related tumors [21]. Subsequent studies came up with the concept of "oncofetal antigens" that are found only at early stages of embryonic and fetal development, but are also present in cancerous tissues [22]. The oncofetal antigens are present in most types of tumors, and embryonic materials were demonstrated to be effective against implantable tumors [23]. This viewpoint has been validated using human embryonic material [24] as Klavins et al. reported that the rabbit antisera against human embryos recognized human tumors, such as lung, colonic and renal cancer. Additional research has also provided evidence that embryonic cells induce the production of antibodies recognizing both tumors and embryos [25]. These results help to support the notion that vaccination with embryonic materials could potentially stimulate the immunity against different types of tumors, probably by recognizing the markers 
shared by cancer cells and embryonic cells.

\subsubsection{Whole-cell cancer vaccines}

Early cancer vaccination strategies focused on using irradiated, autologous tumor cells, which has the advantage of inducing tumor-specific CD4 ${ }^{+}$and CD8 ${ }^{+}$ $\mathrm{T}$ cell responses against multiple tumor-associated antigens (TAAs) [26]. However, this vaccination approach has been hampered by the challenge of collecting large amounts of patient-specific, contaminant-free malignant cells. The efforts to utilize immunostimulatory cytokines as vaccine adjuvants has greatly advanced progress in this area of research. For instance, GVAX is a vaccine composed of irradiated, syngeneic tumor cells genetically engineered to produce the immune stimulatory cytokine granulocyte-macrophage colonystimulating factor (GM-CSF) [27]. GVAX has displayed antitumor capability by promoting specific, robust, and enduring immune responses in preclinical studies either as a monotherapy or in combination with other immunotherapeutic agents. However, two Phase III trials of GVAX for patients with advanced prostate cancer were terminated in 2008 due to a lack of efficacy. Five years later, a Phase II clinical trial testing the effectiveness of GVAX combined with a PD-1 inhibitor to treat pancreatic cancer ended without much success. Although the exact reasons for the lack of success of GVAX in clinical trials are unclear, it is conceivable that potent immune evasion mechanisms in cancerous tissues contributed to the clinical failure of GVAX. Thus, the likelihood of success is highly increased if a whole-cell vaccine is designed to 
block the escape of tumors from immune surveillance.

It is now evident that stem-like cells (cancer stem cells; CSCs) and more differentiated trophoblast-like cells coexist within cancerous tissues [15]. CSCs have been identified in a large number of human malignancies [15]. The presence of CSCs is a particular challenge in developing cancer vaccines, because they play an important role in cancer immune evasion [28]. If CSCs could be targeted in developing cancer vaccines, their antitumor efficacy would be greatly enhanced.

Based on the mechanisms by which CSCs escape from the immune system and antigens shared between fetal tissues and CSCs, vaccines containing embryonic stem cells (ESCs) have been explored for their effectiveness in boosting antitumor immunity. Among them, a unique prophylactic vaccine comprised of irradiated, allogeneic murine ESCs and irradiated murine STO fibroblasts engineered producing GM-CSF has been generated (ESCs/GMCSF vaccine) [29]. This vaccine effectively inhibited the initiation and progression of both implantable and carcinogen-induced lung adenocarcinoma in mice without generating detectable toxicity and autoimmune responses. Importantly, irradiated GM-CSF-expressing fibroblasts alone failed to protect against the outgrowth of lung tumors, indicating that the vaccine efficacy is not elicited by non-specific immune responses against allogeneic, whole cell antigens. The effectiveness of ESCs/GM-CSF vaccine is linked to the generation of robust tumor-specific primary and memory $\mathrm{CD}^{+} \mathrm{T}$ effector 
responses, Th1 cytokine response, decreased tumor-suppressive myeloidderived suppressor cells in the spleen and increased ratio of intratumoral CD8 ${ }^{+}$ T effector/T regulatory cells ( Tregs $)$.

A more recent study lends credence to the idea that undifferentiated stem cells could function as a vaccine against cancer [30]. Kooreman and colleagues have utilized irradiated, syngeneic induced pluripotent stem cells (iPSCs) and the immunostimulatory adjuvant $\mathrm{CpG}$ to generate a stem cell-based vaccine. This vaccine is capable of activating the immune system to recognize the antigens shared between iPSC and cancer cells to confer antitumor immunity. This vaccination strategy not only blocked the outgrowth of implanted primary tumors, but also suppressed the recurrence of resected tumors in an adjuvant setting in mice. Immunization with the iPSC-based vaccine generated effective anti-cancer immune responses by a systemic boost in $\mathrm{CD} 4^{+} \mathrm{T}$ helper and $\mathrm{CD} 8^{+}$ cytotoxic $T$ cell responses. This study provides robust evidence for the idea that embryonic material can function as a potent cancer vaccine.

Similarly, immunization with undifferentiated, pluripotent ESCs in the absence of any immunostimulatory adjuvant only conferred modest antitumor function against implantable colorectal and pulmonary malignancies in mouse models of cancer [25, 31]. All of these studies provide robust evidence for the idea that ESCs with the help of an immunostimulatory adjuvant can function as a potent cancer vaccine against embryonal antigens expressing at early embryonic development stages but not in adult tissues. The key to the success 
of ESC-based cancer vaccines lies in the fact that oncofetal antigens shared by both embryos and malignant cells are potentially immunogenic due to their absence in the 'self' repertoire of humans.

\subsubsection{Peptide cancer vaccines}

Recently, tremendous progress has been achieved towards understanding the unique tumor-associated antigens (TAAs) existing in various tumors. T cells are known to be able to recognize antigen-originated peptide fragments presented by major histocompatibility complex (MHC) molecules at the surface of APCs and subsequently elicit specific immune responses. Thus, the peptide epitopes derived from TAAs have been explored as antitumor therapeutic vaccines [32]. Compared with whole-cell vaccines, peptide-based vaccination strategies hold several advantages, such as inexpensive production of peptides in large quantities at clinical grade, easy administration into cancer patients, and limited toxicity. However, tumor cells are able to acquire various genetic and epigenetic changes during the "escape" phase of cancer immunoediting process, fostering an immunosuppressive tumor microenvironment [33]. This enables malignant cells to alter their antigenic profiles, such as loss of the expression of MHC molecules or immunogenic TAAs, thereby evading the recognition by the immune system. Therefore, a major limitation of peptide-base vaccination is the resistance to immune elimination conferred by the escape of cancer cells from immune recognition. In this scenario, tumor subpopulations lacking MHC molecules or TAAs will be positively selected and propagate as their 
counterparts expressing MHC molecules or TAAs will be eliminated by T cells.

A number of peptide-based cancer vaccines have been developed to utilize recombinant or synthesized epitopes of immunogenic TAAs, and they are designed to stimulate immune responses against one or more TAAs [32]. In the clinic, peptide-based cancer vaccines have generally displayed limited toxicity with only mild side effects reported. Researchers had high hopes for peptidebased cancer vaccination strategies in the beginning. Indeed, immunization with peptide-based cancer vaccines elicits certain degrees of immune responses targeted on tumors, such as production of antitumor cytokines, infiltration of $\mathrm{CD}^{+}{ }^{+}$and $\mathrm{CD} 8^{+}$lymphocytes into malignant tissues, and detection of epitope-specific antibodies in the serum. However, the anti-cancer efficacy of peptide-based cancer vaccines as standalone therapeutics has been largely disappointing during a variety of clinical trials. This failure could be attributed to the immunoediting selection of non-immunogenic tumor cells as well as the strong immunosuppressive microenvironment promoted by cancer cells. Therefore, the current phase of developing peptide-based vaccines is focused on overcoming immunoediting by combination with immunotherapeutic approaches to reverse immunosuppression and enable tumor-specific immune responses. This strategy could potentially unlock the full capability of cancer vaccines and likely provide enduring protection against recurrence of malignancies. 


\subsubsection{Cancer vaccine adjuvants}

For cancer vaccination, specific vaccine antigens are normally used in combination with adjuvants to potentiate and modulate antigen-specific immune responses [34]. Without an adjuvant, a cancer vaccine only provides modest effects from antigens alone. An adjuvant could enhance the efficacy of cancer vaccines through various mechanisms, such as promoting cytokine production, increasing the association between the antigens and APCs, prolonging antigen presence in the blood, and activating antitumor immune cells.

\subsubsection{Cytokines}

Cytokines are a broad group of natural peptides $(\sim 5-20 \mathrm{kDa})$ that function as important immunomodulating agents. Since cytokines are deeply involved in normal immune responses, they hold great promise as potential adjuvants for cancer vaccines [35].

\subsection{Granulocyte-macrophage colony-stimulating factor (GM-CSF)}

Colony-stimulating factors (CSF) are secreted glycoproteins that were discovered by chance in the observation of proliferating granulocytes and macrophages in a tissue culture system [36]. CSFs play an important role in haematopoietic cell activation, survival, differentiation, and proliferation. Granulocyte-macrophage colony-stimulating factor (GM-CSF) is a subtype of CSF produced by macrophages, T cells, mast cells, natural killer (NK) cells, 
endothelial cells, and fibroblasts [37]. GM-CSF stimulates differentiation, proliferation of stem cells, and increases the production of macrophages and dendritic cells necessary for activating $T$ cells. Therefore, GM-CSF is an essential component of the normal immune cascade.

As a growth factor, GM-CSF primarily functions as a multifunctional haematopoietin to promote production of several blood cell lineages [37]. In the case of non-haematopoietic function of GM-CSF, there is still a lot to be discovered. As a key part component in a number of signaling cascades to activate the immune system, GM-CSF can stimulate different signaling pathways in various immune cells to enhance immune responses. For instance, human $\mathrm{T}$ cells can be activated by GM-CSF-mediated increase in MHC-II expression in neutrophils, which is involved in the toll like receptor 4 (TLR4) signaling [38]. In monocytes, the expression of toll-like receptor 2 (TLR2) and tumor necrosis factor alpha (TNF- $\alpha$ ) is stimulated by GM-CSF to initiate host innate immune responses [39]. Moreover, GM-CSF promotes differentiation of dendritic cells in mouse bone marrow [40]. Specifically, GM-CSF activates signal transducer and activator of transcription 5 (STAT5) expression in dendritic cells, which in turn upregulates the cytoplasmic cytokine inducible SH2domain $(\mathrm{CISH})$ protein and promotes subsequent type 1 dendritic cell development [40]. Because of its effect on dendritic cell activation, GM-CSF also plays an important role in differentiation of Th1 type cells, as the expression of MHC-II molecules and immune response in monocytes and dendritic cells 
were enhanced when treated with GM-CSF [41].

The impact of GM-CSF on the immune system makes it critical in different types of diseases related to immune response, particularly cancer. For instance, GM-CSF has been utilized in a dendritic cell-based vaccine against prostate cancer, in which a fusion protein consisting of GM-CSF and the prostate cancer antigen PAP activated patient's dendritic cells before they were reinfused into the patient to stimulate $\mathrm{T}$ cell activation and cancer cell elimination [42]. In another study, GM-CSF and antisense transforming growth factor (TGF)- $\beta 2$ were co-expressed in isolated tumor cells from a patient to generate a autologous cancer vaccine [43]. Similarly, a vaccine against melanoma was designed with a plasmid encoding both GM-CSF and the tumor antigen MAGE1, which can stimulate an antigen-specific immune response through the production of IFN- $\gamma$ and IL-6 [44]. Moreover, GM-CSF along with IL-12 has been tested in clinical trials as a treatment against lung cancer [45].

In many preclinical studies, GM-CSF has been investigated as an adjuvant for prophylactic vaccines protecting against infectious diseases and malignancies. Due to its modulatory role in immune responses suppressing tumor growth, Nemunaitis et al. evaluated a vaccine with autologous tumor cells modified to overexpress GM-CSF in patients with early and advanced stage of NSCLC [46]. Longer survival was observed in the patients vaccinated with GMCSF-expressing tumor cells compared with those immunized with tumor cells only. Furthermore, a preclinical study has shown that GM-CSF is essential for 
anti-cancer efficacy of a prophylactic lung cancer vaccine [29]. Overall, these results provide evidence that GM-CSF possesses the potential as an adjuvant in prophylactic or therapeutic cancer vaccination.

GM-CSF has been extensively explored for its anti-cancer function to stimulate tumor antigen presentation by recruiting dendritic cells and mediate protective immunity by activating $\mathrm{CD} 4^{+}$and $\mathrm{CD} 8^{+} \mathrm{T}$ cells [47]. It is worthy to note that GM-CSF is also capable of stimulating tumor progression under certain circumstances. For instance, GM-CSF appears to enhance proliferation and invasion of lung cancer cells by increasing productions of gelatinases and urokinase plasminogen activator [47]. In mesothelioma patients, GM-CSF produced by tumors stimulates tumor progression through promoting immunosuppression of granulocytes in tumor microenvironments [48]. Therefore, more studies are warranted to elucidate the complexities of GM-CSF clinical applications.

\subsection{IL-23}

IL-23 is a heterodimeric cytokine secreted by activated dendritic cells, which is composed of the IL-12p40 subunit and IL-23p19 subunit [49]. The main function of IL-23 in the immune system is stimulating IFN-y production from $\mathrm{CD}^{+} \mathrm{T}$ cells. Some studies have revealed that IL-23 promotes immunity to target antigens of endogenous antitumor immunity [50]. In another research, the immunological and antitumor effects of IL-23 on gp100-specific vaccination therapy of human melanoma were examined [51]. In this in vivo experiment, systemic IL-23 
administration alone failed to stimulate effective tumor immunity. However, as an adjuvant of gp100 peptide immunization, IL-23 helped suppressing tumor growth through vaccination-induced T cell activation [51]. Moreover, tumorspecific $\mathrm{T}$ cell levels increased in spleen but not in tumor tissues. It is also demonstrated that IL-23 is a potent vaccine adjuvant enhancing intratumoral T cell effector function, rather than increasing intratumoral T cell number for tumor suppression. However, IL-23 produced drastic side effects, including the rapid, systemic, vaccination-independent depletion of nonspecific $\mathrm{CD} 8^{+}$and $\mathrm{CD} 4^{+} \mathrm{T}$ cells. Another toxicity of IL-23 was weight loss caused by reduced appetite. While IL-23 is promising in cancer vaccine adjuvant, further research is required to fulfill its potential by eliminaing the side effects.

\subsubsection{CpG}

First identified in a bacterial genome, CpG is unmethylated deoxycytidyl ("C")deoxyguanosine (“G”) dinucleotides linked by a phosphodiester ("p") bond [52]. Since $\mathrm{CpG}$ motifs have been widely discovered in microbial genomes but rarely identified in vertebrate genomes, they are believed to possess pathogenassociated molecular patterns (PAMPs). It has been found that the pattern recognition receptor toll like receptor 9 (TLR9) in human B cells and plasmacytoid dendritic cells is able to recognize the CpG PAMPs to elicit the innate immune response with the production of Th1 and proinflammatory cytokines. Due to the critical role of plasmacytoid dendritic cells in triggering immune responses required for tumor elimination, it is appealing to utilize CpG- 
based adjuvants for cancer vaccines.

CpG-based cancer vaccine adjuvants are normally designed as synthetic short single-stranded DNA oligodeoxynucleotide molecules containing unmethylated $\mathrm{CpG}$ motifs [52]. As adjuvants for cancer vaccines, CpG oligonucleotides have been shown to enhance activities of professional APCs and generate tumor-specific immune responses in pre-clinical studies. In recent research focusing on an iPSC-based cancer vaccine, CpG oligonucleotides were employed as adjuvants to help suppressing tumor growth in mice [30]. In a prophylactic setting, immunization of CpG and irradiated iPSC inhibited breast cancer, mesothelioma, and melanoma in syngeneic mouse models. Furthermore, this vaccine blocked the recurrence of melanoma at the original resection sites. To further explore the immunity induced by this vaccine, Kooreman and colleagues performed adoptive transfer of iPSC/CpG-primed T cells to immunocompetent mice bearing breast cancer and tumor-experienced lymphocytes to iPSC-inoculated non-obese diabetic severe combined immunodeficiency (NOD-SCID) mice. In both cases, tumor growth was reduced, indicating that $\mathrm{CpG}$ promotes tumor-specific immunity against shared epitopes between iPSCs and cancer cells.

\subsubsection{Others}

Beyond glycoproteins, cytokines, and CpG oligodeoxynucleotides, other adjuvants have been explored $[53,54]$. For instance, mineral salts have also been adopted in vaccine formulations, including insoluble aluminum salts and 
calcium phosphate, which can induce early and long-lasting immunity responses. In addition, bacteria-derived adjuvants, saponin and oil adjuvants have been proved to display different biological and pharmacological activities in cancer immunity induced from vaccination.

\subsection{Exosomes in cancer therapy}

\subsubsection{Biogenesis of exosomes}

Extracellular vesicles (EVs) are a type of membrane capsule released from parental cells and range in size from $20 \mathrm{~nm}$ to $200 \mathrm{~nm}$ in diameter [55]. EVs were firstly found in 1946 as platelet-derived particles by Chargaff and West [56]. After a series of studies, EVs were discovered to perform numerous essential functions in intercellular communication [57-59]. In multicellular organisms, they can be found in nearly all tissues and body fluids [60]. EVs regulate physiological and pathological functions by transferring their cargo, such as proteins and nucleic acids, between cells. Among different subtypes of EVs, exosomes are small membrane vesicles released from various cell types into the extracellular space upon fusion of intermediate endocytic compartments, multivesicular bodies (MVBs), with the plasma membrane [61].

\subsubsection{Composition of exosomes}

As a subtype of EVs, exosomes have spherical or cup-shaped construction and their size is normally in the range of $30-100 \mathrm{~nm}$ in diameter. The existence and biological function of exosomes have been investigated since the mid-20th 
century [55]. It is now known that exosomes inherit biological functions from their own parental cells, as they contain biological materials acquired from the cytosol, including proteins, RNA, double-stranded DNA, and metabolites [62]. These exosomal molecules are either located inside exosomes or associated with their membrane. Exosomes normally contain general as well as cell typespecific proteins with various bioactivities. The protein profiles of exosomes are similar to those identified in the endocytic pathway, plasma membrane, or cytosol [61]. A select set of functional coding (mRNAs) or non-coding (microRNAs) nucleic acids are also enriched in exosomes. It is hypothesized that the presence of double-stranded DNA in exosomes from certain types of cells, particularly cancer cells, could serve as a biomarker [62]. On a similar note, the metabolomic profiles of exosomes circulating in blood and other biofluids also display diagnostic potential in detecting various diseases, including cancer [61]. The composition of exosomes could be examined by various biological techniques, such as western blotting, flow cytometry, and spectrometry.

\subsubsection{Functions of exosomes in tumorigenesis}

Tumors are composed of a heterogeneous population of malignant cells along with resident and infiltrating non-transformed cells [63]. Dynamic interactions between cancer cells and non-malignant cells are critically involved in every stage of carcinogenesis, as well as responses to cancer therapies. As major conveyors of intercellular communication, exosomes play an important role in 
the crosstalk between cancer cells and other types of cells in malignant tissues, particularly immune cells infiltrating into tumors [64].

Emerging evidence indicates that tumor-derived exosomes profoundly influence the functions of intratumoral immune cells. In some cases, exosomes derived from tumors enhance immunity against cancer. For instance, exosomes derived from melanoma tumors could be recognized by $\mathrm{T}$ cells through the endogenous tumor antigens present on exosomes [65]. Furthermore, exosomal tumor antigens could be transferred to dendritic cells to elicit robust CD8 ${ }^{+} \mathrm{T}$ celldependent antitumor immune response in syngeneic and allogeneic mouse tumor models [66]. On the other hand, tumor-derived exosomes are also capable of boosting pro-tumor immune signaling [67]. One example is that exosomes of a variety of tumors promote tumor-associated macrophages known to stimulate cancer progression and metastasis.

Immunocyte-derived exosomes also play a multifaceted role in regulating tumor immunity, rather than just inhibiting or promoting malignancies [68]. Exosomes released from antitumorigenic immune cells often block carcinogenesis [68]. Like their parental cells, exosomes originating from dendritic cells possess high levels of transmembrane TNF, Fas ligand (FasL), and TNF-related apoptosis-inducing ligand (TRAIL) that induce apoptotic signaling in a variety of tumors [69]. Furthermore, NK cells are also activated by exosomes of dendritic cells to produce antitumor cytokines [69]. On a similar note, exosomes derived from NK cells are enriched in FasL and perforin, which 
eliminate human melanoma cells but not unmalignant cells [70]. In contrast, exosomes derived from pro-tumor immune cells, such as tumor-associated macrophages, normally promote tumor evasion from immune surveillance [68].

\subsubsection{Exosome-based cancer vaccines}

Since tumor-associated antigens or factors stimulating tumor-specific immune responses are encapsulated in the exosomes from either malignant cells or certain types of immunocytes, numerous investigations have explored exosomes as an anti-cancer vaccination strategy [68]. The first study about the role of exosomes in immunotherapy was conducted in the 1990s, in which the exosomes isolated from B cells were found to stimulate an antigen-specific $T$ cell response [71]. Subsequent in-depth characterization of exosomes at the molecular level and standardized exosome isolation and storage protocols have greatly advanced pre-clinical and clinical studies of exosome-based cancer vaccines, which display significant advantages over their cell-based counterparts. Among exosomes derived from different sources, those originated from dendritic cells and tumor cells have drawn notable interest due to their particular biological functions to promote tumor-specific immune response.

Dendritic cell-derived exosomes (Dexosomes) inherit the ability to initiate an antigen-specific immune response from their parental cells [72]. These exosomes are enriched in peptide-MHC-I and peptide-MHC-II complexes that 
are 10-100 times more abundant than those in dendritic cells. In addition, Dexosomes harbor co-stimulatory factors (e.g. CD40, CD80, and CD86) as well as other molecules involved in immune responses. Pre-clinical research has demonstrated that Dexosomes elicit robust antitumor immune responses that activate cytotoxic T cells and NK cells in vitro and in vivo [72], which paved the way for human clinical trials of Dexosomes as a cell-free cancer vaccine. Two phase I clinical trials and one phase II clinical trial of Dexosomes have been carried out in patients with advanced malignancies. In the first phase I clinical trial with melanoma patients, exosomes from autologous dendritic cells pulsed with melanoma-associated antigen (MAGE) peptides were utilized to immunize stage III/IV melanoma patients. The safety of exosome inoculation was examined and tumor-specific $T$ cell responses was determined [73]. The second phase I clinical trial tested a vaccine composed of Dexosomes loaded with the MAGE tumor antigens in patients with advanced NSCLC [74]. This Dexosome-based vaccine evoked immune responses in some patients and exhibited limited toxicity, but its antitumor efficacy appeared to be modest. Following up the phase I clinical trials, a second generation of Dexosome-based cancer vaccine was developed [75]. The new vaccine was composed of exosomes derived from TLR4L- or interferon (IFN)- $\gamma$-maturated dendritic cells that elicited more robust $\mathrm{T}$ cell stimulation compared with their counterparts from immature dendritic cells. This phase II trial of patients with advanced NSCLC improved upon the limited $\mathrm{T}$ cell-mediated immune responses 
observed in the phase I studies and enhanced the capacity of Dexosomes to boost NK cell-mediated antitumor immunity. Overall, these clinical trials have demonstrated the feasibility and safety of Dexosome-based vaccine, as well as the ability of Dexosomes to evoke T cell- and NK cell-based immune responses in patients with advance malignancies.

Tumor-derived exosomes (Texosomes) have been considered as a specific stimulus for antitumor immunity, since they are enriched in MHC-I molecules, antigens, and heat shock proteins (HSPs) that could increase immune responses, particularly cytotoxic T cell activation [76], [77]. More importantly, Texosomes evoke immune responses against tumors more effectively than either irradiated live cancer cells or their lysates [78]. Since Texosomes are capable of transferring a selection of tumor antigens to dendritic cells, they have been explored as the carriers for tumor antigens in cancer immunotherapy [68]. Although Texosomes could potentially serve as a cell-free cancer vaccine, in vitro expansion of purified autologous malignant cells from patients is challenging in clinical settings. It has been shown that large amount of Texosomes are present in the biological fluids of patients, such as ascitic fluid [78]. Due to their high abundance, Texosomes isolated from the ascitic fluid of patients with advanced colorectal carcinoma (CRC) were investigated for their potential as a cancer vaccine [79]. Like Texosomes from other sources, ascitesderived Texosomes were enriched in MHC-I and MHC-II molecules, HSPs (e.g. HSC70, HSP70, and HSP90), co-stimulatory molecules, intercellular cell- 
adhesion molecule-1 (ICAM-1), and the classical carcinoembryonic antigen (CEA) of CRC. A phase I clinical trial was conducted with ascites-derived Texosomes in the absence of presence of immunostimulatory factor GM-CSF [79]. This vaccination strategy was safe and well-tolerated in CRC patients. Importantly, ascites-derived Texosomes along with GM-CSF, but not ascitesderived Texosomes alone, induced beneficial CEA-specific antitumor cytotoxic T lymphocyte (CTL) response, demonstrating its potential as a cancer vaccine candidate.

However, it is noteworthy that the Texosomes from some tumor cells promote tumor growth and angiogenesis [80], as they contain a large part of a protein repertoire to suppress immune responses or exacerbate tumor cell proliferation by different molecules. Moreover, Tumor-derived exosomes can induce resistance to the immune response by decreasing antitumor T cells $[78$, 81]. The utilization of Texosomes as tumor vaccines depends on the types of malignancies and the immunogenicity of their antigens. Thus, further research is needed to unlock the immunogenic potential of Texosomes.

\subsection{Hypothesis and significance of the project}

Numerous studies have focused on prophylactic vaccines against diseases, since they can decrease or even eradicate a disease around the world. For infectious disease control, vaccination is effective, because the pathogen can be recognized by the immune system after the vaccine provides actively acquired immunity. However, tumors have not been conquered by antitumor 
vaccination, because tumors cells are recognized as "self" by the immune system. As a result of tumor cell escaping from immune surveillance, tumors continue developing and metastasizing. To date, a number of vaccination strategies stimulating tumor-specific immune responses have been explored as immunotherapy against cancer, among which tumor cell lysates and tumor antigens have been extensively studied [82]. However, the therapeutic effects of most antitumor vaccines are not significant in clinical trials. The only FDAapproved cancer treatment vaccine is Sipuleucel-T that treats metastatic prostate cancer patients with limited improvement of survival [83].

ESCs display the characteristics of self-renewing and they are multipotent cells. A vaccine comprised of allogeneic murine ESCs and murine fibroblasts expressing the immunostimulatory factor GM-CSF successfully blocked the outgrowth of lung tumors in different mouse models [29]. However, an intact ESC-based cancer vaccine raises ethical concerns and the potential risks of inducing teratomas and autoimmunity. Exosomes isolated from ESCs inherit some functions of ESCs as they possess the antigens of ESCs. Because of the similarity of antigens between ESCs and tumor cells, it is possible that ESCderived exosomes could serve as a cancer vaccine to suppress tumor growth. Therefore, we hypothesized that the exosomes derived from ESCs would function as a novel cell-free vaccine to effectively prevent lung tumor development in mice. The success of this study will have important implications for lung cancer prevention and could potentially lead to clinical trials of a similar 
human vaccine.

\section{CONCLUSION}

Embryonic materials have a long history of being studied as a preventive strategy against cancer in animal experiments due to their antigenic similarities to tumor cells. However, the prophylactic function of ESCs against cancer is still under investigation. Given that exosomes hold great promise as diagnostic biomarkers and therapeutic vehicles, using ESC-derived exosomes as a cancer vaccine possesses high potential to prevent lung cancer development. The experiments proposed in this study will address significant unanswered questions pertaining to the potential of ESC-derived exosomes as an immuneprophylactic agent for lung cancer. 


\section{CHAPTER II}

\section{EXOSOMES DERIVED FROM MURINE EMBRYONIC STEM CELLS OVER-}

EXPRESSING GM-CSF PREVENT TRANSPLANTED LUNG TUMOR

\section{DEVELOPMENT}

\section{INTRODUCTION}

An early hypothesis of tumorigenesis suggests that mutations in undifferentiated progenitor cells give rise to malignant cells that are capable of both self-renewal and differentiation. Interestingly, this theory essentially predicts the discovery of cancer-initiating cells (CICs) that came over 100 years later. In the mid-1960s, tumor cells and embryonic stem cells were shown to possess common gene products such as the oncofetal antigens [84]. During the next decade, a large number of studies confirmed these findings and revealed that embryonic antigens are re-expressed in malignant cells from a number of different tissues and that vaccination of animals with fetal material can prevent the outgrowth of tumors [18]. It now appears that most, if not all, types of neoplastic cells express certain embryonal antigens, termed as "retrogenetic expression" [19]. Many of these embryonic gene products (also called carcinoembryonic antigens) are not expressed in the adults and thus, are 
not included in the repertoire of 'self' during the process of thymic selection that occurs near the end of gestation. Such 'non-self' embryonic antigens are immunogenic and can prime the immune cells to mount an anti-tumor response [85]. Exploiting such embryonic antigen immunogenicity, we have designed a unique stem cell-based vaccine that stimulates the immune system to recognize shared oncofetal antigens and confers protection against tumors.

In our initial attempts to produce a prophylactic vaccine, we successfully combined irradiated allogeneic murine embryonic stem cells (ESCs) with murine STO fibroblasts expressing GM-CSF (STO-GM) as an immune stimulant (ES cell vaccine) [29]. We discovered that ES cell vaccination was 70-100\% effective in preventing both implanted and carcinogen induced lung adenocarcinomas.[29] Vaccinated mice remained tumor-free over a 90-day observation period. A very recent study by Kooreman et al. [30] supports our earlier results albeit with a different form of pluripotent stem cells (induced pluripotent stem cells; iPSCs) and a different adjuvant (CpG) and lends further credence to the theory that embryonic material can be an effective prophylactic vaccine against "other" non-mutated neo-antigens, i.e., tumor antigens derived from proteins that are only expressed during embryonic development and not in adult tissues.

Although the ES cell vaccine holds promise for inducing anti-lung cancer immune responses, such an approach has two obvious problems in terms of application to humans: (i) The use of whole ESCs—although irradiated—raises 
the risk of generating embryomas/teratomas. (ii) Using murine fibroblasts to generate GM-CSF is needlessly complicated. To overcome these challenges, we have attempted to develop an alternative cell-free prophylactic vaccine to avoid the need for the administration of intact ESCs.

Exosomes are cell-derived nanovesicles [86] (30-100nm) that have recently gained renewed interest as they possess immense potential for cancer therapy [87]. Within the cells, invagination and budding from the limiting membrane of late endosomes, results in formation of vesicles that contain cytosol and the extracellular domain of transferrin receptors at their surface. These internal vesicles (called exosomes) are then secreted into extracellular environment following the fusion of multivesicular endosomes with the plasma membrane [88]. In vitro and in vivo studies suggest that exosomes can bind to target-cell membranes, or can fuse with target cells to facilitate the exchange of membrane proteins and cytosol between two cell types [89]. Importantly, exosomes are also capable of transferring nucleic acids, such as mRNA and microRNA, which represent a new paradigm of genetic exchange between cells $[89,90]$. Recent studies indicate that exosomes can operate as potential immunotherapeutic agents, with promising results in pre-clinical studies of cancer immunotherapy [87]. Exosomes have several advantages over cell-based therapies, including high bio-availability, bio-stability, and lower costs [91, 92]. Since exosomes can deliver large amounts of cargo directly to target cells, this property can be exploited to include therapeutics as well as 
immunostimulatory adjuvants in engineered exosomes [93].

In this study, we first generated murine ESCs engineered to produce GMCSF in amounts similar to those produced by STO fibroblasts in our earlier study. Then we purified ESC-derived exosomes (ES-exo), thereby producing a self-contained, relatively stable exosome-based vaccine. Here we show that, prophylactic vaccination of mice with ESC-exosomes expressing GM-CSF (ESexo/GM-CSF) is very effective in preventing implantable lung tumors with no detectable toxicity. Importantly, anti-tumor efficacy of the ES-exo/GM-CSF vaccine is associated with robust $\mathrm{CD} 8^{+} \mathrm{T}$ effector responses and infiltration of $\mathrm{CD}^{+} \mathrm{T}$ cells into the tumor, leading to increased intratumoral $\mathrm{CD} 8^{+} \mathrm{T}$ effector/T regulatory ( $\left.T_{\text {regs }}\right)$ cell ratio in the tumors. Collectively, our findings provide a strong rationale for further developing this novel cell-free exosome-based vaccination strategy for the prevention of cancer. 


\section{MATERIALS AND METHODS}

\section{Cell culturing}

Murine embryonic stem cell line ES-D3 was purchased from ATCC (CRL-11632; Manassas, VA). To remove exosomes, fetal bovine serum (FBS; SCRR-302020; ATCC) was centrifuged at $100,000 \times \mathrm{g}$ for 16 hours at $4^{\circ} \mathrm{C}$ by a $45 \mathrm{Ti}$ rotor in an ultracentrifuge (OptimaTm L-100XP, Beckman Coulter; Brea, CA). After centrifugation, serum supernatant was used as exosome-free FBS to culture ES-D3 cells for exosome preparation. ES-D3 cells were grown in KnockOut ${ }^{\mathrm{TM}}$ Dulbecco's Modified Eagle's Medium (10-829-018; Thermo Fisher; Waltham, MA) supplemented with $15 \%$ exosome-free FBS, $50 \mathrm{U} / \mathrm{ml}$ penicillin (sc45000652; Mediatech; Manassas, VA), $50 \mu \mathrm{g} / \mathrm{ml}$ streptomycin (sc45000-652; Mediatech), $0.1 \mathrm{mM}$ non-essential amino acids (SH3023801; Thermo Fisher), 2 mM L-glutamine (L0131-0100; VWR; Radnor, PA), $0.1 \mathrm{mM} \quad \beta$ mercaptoethanol (21985023; Thermo Fisher) and 100 units/ml leukemia inhibitory factor (LIF; ESG1106; Thermo Fisher). ES-D3 cells were cultured in $15-\mathrm{cm}$ tissue culture dishes pre-coated with $0.1 \%$ gelatin (ES006B; Thermo Fisher) for 30 minutes without feeder layer cells as described before[94]. Murine Lewis lung carcinoma (LLC) cell line was obtained from ATCC (CRL-1642). Murine mammary carcinoma cell line 4T1 was also acquired from ATCC (CRL2539). LLC and $4 T 1$ cells were cultured in medium containing Dulbecco's modified eagle's medium (DMEM; SH30243.01; Mediatech), 10\% FBS (900108; Gemini; Broderick, CA), 100 units/ml penicillin (Mediatech) and $100 \mu \mathrm{g} / \mathrm{ml}$ streptomycin (Mediatech). ES-D3 cells, LLC cells and 4T1 cells were cultured in a $5 \% \mathrm{CO}_{2}$ humidified incubator at $37^{\circ} \mathrm{C}$ and were cultured no longer than $6-$ 
8 weeks before a new vial of cryopreserved cells were thawed. All of our cryopreserved stocks were prepared at passage two after receipt from ATCC and were authenticated by ATCC cell bank using the Short Tandem Repeat (STR) profiling.

\section{Plasmids}

To generate the plasmid expressing murine GM-CSF cDNA in ES-D3 cells, the transfection plasmid pEF1 $\alpha$-FD3ER-IRES-hrGFP was acquired from Addgene (37270; Cambridge, MA). In this plasmid, human polypeptide chain elongation factor $1 \alpha(E F 1 \alpha)$ promoter drives the target CDNA expression along with the marker protein humanized Renilla reniformis GFP (hrGFP) [95, 96]. pEF1aFD3ER-IRES-hrGFP was first digested with EcoRI to produce the vector backbone. While self-ligation of the vector backbone produced the empty expression vector pEF1a-IRES-hrGFP, inserting murine GM-CSF cDNA into the vector backbone generated pEF1a-mGM-CSF-IRES-hrGFP. All of the plasmids were sequenced to confirm their identities.

\section{Exogenous expression of GM-CSF}

ES-D3 cells were transfected with pEF1a-mGM-CSF-IRES-hrGFP or its control counterpart pEF1 $\alpha$-IRES-hrGFP along with the plasmid pBabe-Neo (1767; Addgene). pBabe-Neo was co-transfected into ES-D3 cells to facilitate selection of stably transfected cells. Briefly, $1.4 \times 10^{6}$ ES-D3 cells were cultured in a gelatin-coated $10-\mathrm{cm}$ tissue culture dish with $10 \mathrm{ml}$ medium for 24 hours, and two dishes of ES-D3 cells were prepared for transfection. Two plasmid mixtures were transfected into ES-D3 cells: \#1: pEF1a-IRES-hrGFP vector and pBabe-Neo; \#2: pEF1a-mGM-CSF-IRES-hrGFP and pBabe-Neo. 
Transfection was carried out using Lipofectamine $2000 \AA$ transfection reagent (11668019; Thermo Fisher) following the manufacturer's protocol. Twenty-four hours after transfection, transfected cells were split into two 10-cm dishes and cultured with neomycin $(0.5 \mathrm{mg} / \mathrm{ml})$ to eliminate untransfected cells. The transfected cells were continuously cultured in the medium containing 0.5 $\mathrm{mg} / \mathrm{ml}$ neomycin for 2 weeks to generate bulk population of stably transfected ES-D3 cells.

GFP-positive ES-D3 cells were acquired by flow cytometry sorting (MoFlo; Beckman Coulter). A single GFP-positive ES-D3 cell was plated into one well of a gelatin-coated 96-well tissue culture plate containing $1 \times 10^{3}$ parental ESD3 cells in neomycin-free medium. Co-culturing transfected ES-D3 cells with their untransfected parental counterparts ensures that stably transfected single ES-D3 cells survive and proliferate as a single clone. Forty-eight hours later, neomycin was added to 96 -well plates with the final concentration of $0.5 \mathrm{mg} / \mathrm{ml}$ to eliminate untransfected parental ES-D3 cells. Once GFP-positive ES-D3 single clones in 96-well tissue grew confluence, they were transferred to gelatin-coated $6-\mathrm{cm}$ tissue culture dishes. Clonal ES-D3 cell lines were evaluated for their GFP fluorescence intensity by flow cytometry (FACScalibur; Beckon Dickinson; Franklin Lakes, NJ). ES-D3 clones expressing either GMCSF or the empty vector with high levels of green fluorescence were identified.

\section{Evaluation of pluripotency}

The pluripotency of ES-D3 cells was evaluated using a Stemflow ${ }^{\mathrm{TM}}$ Human and Mouse Pluripotent Stem Cell Analysis Kit (BDB560477; Beckon Dickinson) following manufacturer's protocol. The expression of different pluripotency 
markers, including SSEA-1, Oct-3/4 and SSEA-4, was measured using flow cytometry (FACScalibur; Beckon Dickinson).

\section{Isolation of exosomes}

ES-D3 cells $\left(10 \times 10^{6} /\right.$ plate $)$ were plated in $15-\mathrm{cm}$ tissue culture plates precoated with $0.1 \%$ gelatin with $15 \mathrm{ml}$ exosome-free medium per plate. After culturing for 72 hours, medium was collected and stored at $4^{\circ} \mathrm{C}$ up to 1 week without compromising exosome integrity. The collected medium was centrifuged at $5,000 \times \mathrm{g}$ for 60 minutes at $4^{\circ} \mathrm{C}$ in a $\mathrm{JA}-10$ rotor using an $\mathrm{Avanti} 囚$ J-26XPI centrifuge (Beckman Coulter). After centrifugation, the supernatant was collected and transferred into polycarbonate bottles (355622; Beckman Coulter) for subsequent ultracentrifugation. The ultracentrifugation was carried out at $100,000 \times \mathrm{g}$ for 90 minutes at $4^{\circ} \mathrm{C}$ using a $45 \mathrm{Ti}$ rotor with an ultracentrifuge (OptimaTm L-100XP, Beckman Coulter). After ultracentrifugation, the supernatant was removed without disturbing the exosomal pellets. The tight pellets at the bottom of the centrifuge tubes were gently washed twice with $1 \mathrm{ml}$ of $1 \times$ PBS to remove any traces of medium. Then pellets were resuspended in 1 x PBS, and exosome preparations were quantitated by their protein concentrations using BCA (bicinchoninic acid) assay (23223; Thermo Fisher). Generally, exosomes containing $4 \mu \mathrm{g}$ protein can be acquired from $1 \mathrm{ml}$ of ES-D3 culturing medium. The exosomal preparations were resuspended at a concentration of $5 \mu \mathrm{g} / \mu \mathrm{l}$ in $1 \times$ PBS and stored at $-80^{\circ} \mathrm{C}$.

\section{Transmission electron microscopy (TEM)}

To fix the exosomes, samples (3-5 $\mu \mathrm{g} / \mu \mathrm{l})$ were incubated with $2 \%$ EM grade 
paraformaldehyde (15710; Electron Microscopy Sciences; Hatfield, PA) at room temperature for 2 hours. Then fixed exosomes (10 $\mu$ l) were loaded on Cu grids with carbon support film (FF200-Cu; Electron Microscopy Sciences). To stain the exosomal samples, UranyLess staining solution (22409; Electron Microscopy Sciences) was dropped on the Cu grids with exosomes for 1 minute at room temperature as suggested by the manufacturer. After being drained with a filter paper, the grids were dried overnight at room temperature for TEM image acquisition. A transmission electron microscope (HT7700; Hitachi; Santa Clara, CA) was used to acquire TEM images.

\section{Evaluating exosomes by western blot analysis}

Exosomes were resuspended in $1 \times$ SDS-PAGE at a concentration of $1.2 \mathrm{mg} / \mathrm{ml}$. Exosome lysates $(10 \mu \mathrm{l})$ were loaded into each well in a 4-20\% Bis-Tris gel (M42015; GenScript; Piscataway, NJ) and transferred onto PVDF membrane (IPVH00010; EMD Millipore; Burlington, MA). The membrane was incubated with appropriate primary and secondary antibodies in blotting buffer $(1 \times$ PBS with $0.2 \%$ Tween-20) supplemented with $10 \%$ (w/v) non-fat dry milk (NC9022655; Thermo Fisher).

Proteins were detected using an enhanced chemiluminescence detection system (32106, Thermo Fisher). Antibodies (Abs) for western blot were: antiAlix mAb (clone AC-15, sc-53540); anti-Annexin V mAb (clone H-3, sc-74438); anti-CD81 mAb (clone B-11, sc-166029); anti-Flotillin-1 (clone C-2; sc-74566); anti-CD86 mAb (clone D-6, sc- 28347), anti-cytochrome c mAb (clone A-8, sc13156) from Santa Cruz Biotechnology (Dallas, TX), anti-calnexin pAb (ADISPA-860); anti-protein disulfide isomerase (PDI) pAb (ADI-SPA-890) from Enzo 
(Farmingdale, NY), anti-GAPDH pAb (600-401-A33S) from Rockland (Limerick, PA), anti-Oxphos COX IV-subunit IV mAb (clone 20E8C12; A21348); peroxidase-conjugated goat anti-rabbit $\lg$ (31460); peroxidase-conjugated goat anti-mouse lgG (31430) from Thermo Fisher.

\section{ELISA measurement of GM-CSF}

To determine GM-CSF protein concentration, a murine GM-CSF ELISA kit (88733422; Thermo Fisher) was used following the manufacturer's protocol. After treated in $100 \mu \mathrm{l}$ of $1 \times$ PBS with $0.05 \%$ Tween-20 at room temperature for 30 minutes, ESD3 cell culturing medium was added to an ELISA plate coated with capture antibody at $4^{\circ} \mathrm{C}$ overnight and washed with $1 \times$ PBS $+0.05 \%$ Tween-20 buffer. Following incubation with detection antibody and avidin-HRP, the absorbance at $450 \mathrm{nM}$ was determined using a microplate reader (PowerWave XS; BioTek; Winooski, VT). To test the GM-CSF location in exosome, GM-CSF protein levels in exosomes were determined using the same ELISA protocol but with slight modifications. Briefly, exosomes $(0.6 \mu \mathrm{g})$ were pre-treated in $100 \mu$ l of PBS with or without $0.05 \%$ Tween-20 at room temperature for $30 \mathrm{~min}$. Next, samples were added to an ELISA plate coated with capture antibody and washed with either PBS alone or PBS $+0.05 \%$ Tween-20 buffer. Following incubation with detection antibody and avidin-HRP, the absorbance at $450 \mathrm{nM}$ was determined using a microplate reader (PowerWave XS; BioTek).

\section{Mice}

Wild-type male C57BL/6 mice and female Balb/c mice were purchased from Jackson Laboratory (Bar Harbor, ME). Mice were housed at the University of 
Louisville Research Resources Facilities (RRF), which has the American Association for the Accreditation of Laboratory Animal Care (AAALAC) Accreditation and the Office for Protection from Research Risks (OPRR) IACUC Assurance. The facility also strictly follows the "Guide for the Care and Use of Laboratory Animals" (Institute of Laboratory Animal Resources, National Research Council, National Academy Press, 1996). The experiments involving mice were approved by University of Louisville IACUC (protocol number: 18301).

\section{Vaccination and implanted tumor challenge}

Male C57BL/6 mice and female Balb/c mice (6-8 weeks of age, 8 mice/group) were immunized twice (days 0 and 7 ) with vehicle only ( 1 x PBS, $100 \mu l / m o u s e)$, $225 \mu \mathrm{g}$ exosomes isolated from ES-D3 cells expressing the empty vector (ESexo) or $225 \mu \mathrm{g}$ exosomes isolated from ES-D3 cells stably expressing GM-CSF (ES-exo/GM-CSF). Mice were injected with $1 \times$ PBS or exosomes by subcutaneously (s.c.) in the right flank. On day 14 , following vaccination, mice were challenged with s.c. inoculation of $\operatorname{LLC}\left(0.1 \times 10^{6}\right)$ or $4 \mathrm{~T} 1\left(0.1 \times 10^{6}\right)$ in the left flank. Tumor growth was monitored by measuring tumor width $(\mathrm{W})$ and length $(\mathrm{L})$ in $\mathrm{mm}$ using calipers every other day and tumor volumes $(\mathrm{V})$ in $\mathrm{mm}^{3}$ were calculated by the following formula: $V=\left(W^{*} W^{*} L\right) / 2$.

\section{Evaluation of Fertility}

Female C57BL/6 mice (8 weeks of age, 4 mice/group) were immunized twice (days 0 and 7 ) with vehicle control ( 1 x PBS, $100 \mu \mathrm{l} /$ mouse) or $225 \mu \mathrm{g}$ exosomes containing GM-CSF (ES-exo/GM-CSF). The mice were subjected to s.c. inoculation of vehicle control or exosomes in the right flank. Following two 
weekly vaccinations, each of the female mice from control or vaccination group was bred with one male C57BL/6 mouse (10 weeks of age). The litter size and gender ratio were evaluated after newly born mice reached 4 weeks of age.

\section{Evaluation of toxicological effects}

Female C57BL/6 mice ( 8 weeks of age, 4 mice/group) were subjected to two weekly vaccinations with vehicle only ( 1 x PBS, $100 \mu \mathrm{l} /$ mouse), $225 \mu \mathrm{g}$ control exosomes (ES-exo) or $225 \mu \mathrm{g}$ exosomes containing GM-CSF (ES-exo/GMCSF). One week after completing the second vaccination, whole blood was drawn from mice by cardiac puncture immediately following $\mathrm{CO}_{2}$ asphyxiation. To examine liver and kidney function, whole blood was transferred to serum separator tubes (365967; Beckon Dickinson). One hour post-collection, samples were centrifuged for 10 minutes at $10,000 \times \mathrm{g}$, and serum was collected from each sample and analyzed by the Research Resource Center (RRC) facility at the University of Louisville for non-steroidal anti-inflammatory

drug (NSAID) toxicological analysis. While the liver function was evaluated by measuring alanine transaminase (ALT) and alkaline phosphatase (ALP) levels, kidney damage was assessed by levels of creatinine and blood urea nitrogen (BUN). Simultaneously, whole blood was collected in EDTA-coated tubes (365974; Beckon Dickinson) and analyzed by the RRC facility for complete blood count $(\mathrm{CBC})$ analysis.

\section{Flow cytometric analysis.}

Single cell suspensions from spleen were stained with relevant antibodies for 30 minutes after blocking with anti-CD16/CD32 antibody for 15 minutes at $4{ }^{\circ} \mathrm{C}$. After washing, cell surface and intracellularly stained cells were analyzed on a 
FACSCalibur (Becton Dickinson) and results were analyzed with FlowJo software (TreeStar, Inc., Ashland, OR). Antibodies used for evaluating splenocytes were: anti-CD3 mAb (clone 17A2, 100222); anti-CD4 mAb (clone GK1.5; 100406); anti-CD8 mAb (clone 53-6.7; 100712); anti-CD11b mAb (clone M1/70; 101228); anti-Ly6C mAb (clone HK1.4; 128006); anti-Ly6G mAb (clone 1A8; 127608); anti-CD16/CD32 mAb (clone 2.4G2; 101320) from Biolegend (San Diego; CA), anti-CD44 mAb (clone IM7; 17-0441-82); anti-CD25 mAb (clone PC61.5; 45-0251-82) from (Thermo Fisher), anti-CD62L mAb (clone MEL-14; 553152; BD Biosciences; San Jose, CA).

\section{Intracellular cytokine staining}

Spleens were isolated from mice in different treatment groups 10 days after the second vaccination. Splenocytes were stimulated with LLC lysate $(50 \mathrm{mg} / \mathrm{ml})$ or $4 \mathrm{~T} 1$ lysate $(50 \mathrm{mg} / \mathrm{ml})$ for 4 days. For TNF- $\alpha$ and IFN- $\gamma$ production, effector cells were harvested and restimulated for 6 hours with respective tumor cell lysate in the presence of Golgiplug (555029; BD Biosciences) at a concentration of $1 \mu \mathrm{l} / \mathrm{ml}$ of culture medium. After restimulation, cells were harvested and Fc receptors were blocked using anti-CD16/CD32 antibody. Then, cells were stained for surface expression of CD8 and intracellular expression of cytokines using Cytofix/Cytoperm kit (555029; BD biosciences) according to the manufacturer's instructions and analyzed by flow cytometry.

\section{Analysis of tumor-infiltrating $T$ cells}

Vaccinated and control mice bearing LLC tumors were euthanized 18-21 days after tumor challenge. Solid tumors were dissected, chopped into small pieces using surgical scissors, and enzymatically dissociated in HBSS containing 400 
$\mathrm{U} / \mathrm{ml}$ collagenase type IV (C9891, Sigma-Aldrich), $0.025 \mathrm{mg} / \mathrm{ml}$ hyaluronidase (H6254, Sigma-Aldrich), $0.01 \mathrm{mg} / \mathrm{ml}$ DNase I (D5025, Sigma-Aldrich) for 2 hours at $37^{\circ} \mathrm{C}$ with occasional shaking. The resultant cells were washed and passed through a Ficoll gradient (17144002; GE Healthcare; Chicago, IL) to eliminate dead cells. Anti-CD45 antibody was used to selectively exclude $\mathrm{CD}^{-} 5^{-}$tumor cells from analysis. Tumor infiltrating lymphocytes (TILs) were then analyzed by flow cytometry for the expression of CD4, CD8, and CD25 markers. Tregs $\left(\right.$ Foxp $\left.3^{+}\right)$were analyzed using the anti-mouse Foxp3 staining kit (00-5523-00; eBioscience). The same number of cells (based on side-scatter and forward-scatter analyses) was acquired in all samples. For intracellular IFN- $\gamma$ and TNF- $\alpha$ analysis, TILs were stimulated with LLC lysates $(50 \mathrm{mg} / \mathrm{ml})$ for 24 hours and restimulated with LLC lysates for 6 hours in the presence of Brefeldin A. After restimulation, cells were harvested, treated with Fc block, and stained for surface expression of CD8 and intracellular expression of cytokines using Cytofix/Cytoperm kit according to the manufacturer's instructions (555029; BD Biosciences) and analyzed by flow cytometry.

\section{Statistical analysis}

StatView version 5.0.1 software (Windows version; SAS Institute, Cary, NC) or GraphPad Prism 5.0 software (GraphPad Prisim Software, Inc., La Jolla, CA) were used for all statistical analyses. Comparisons between groups were done using Student's $t$ test or one-way analysis of variance (ANOVA) where appropriate. Survival curves were analyzed using the log-rank test. Statistical significance was assumed at $p<0.05$. 


\section{RESULTS}

\section{Exogenous expression of GM-CSF in murine embryonic stem cell ES- D3.}

The antigenic similarity between embryos and tumors has raised the idea of using embryonic material as a prophylactic vaccine against cancer [19, 97, 98]. An earlier study reported that a vaccine comprised of allogeneic murine ESCs and murine STO fibroblasts expressing GM-CSF to amplify immune responses successfully blocked the outgrowth of different lung tumors in mice [29]. However, this vaccine is impractical for humans for at least two reasons. First, administering intact, live ESCs carries the risk of generating embryomas/teratomas in MHC-matched recipients. Second, the use of live fibroblasts to produce GM-CSF is unnecessarily complicated. We sought to overcome these hurdles by developing an alternative prophylactic vaccine comprised of exosomes derived from murine ESCs expressing GM-CSF (Figure 2.1). To this end, we first attempted to stably over-express exogenous murine GM-CSF in murine ESC cell line ES-D3 (Figure 2.2).

Previous studies have shown that the cellular elongation factor-1a (EF1a) promoter efficiently drives exogenous gene expression in murine ESCs [99, 100]. Murine GM-CSF cDNA was cloned into the transfection vector pEF1aIRES-hrGFP to generate a corresponding GM-CSF expression vector (Figure 2.2A). This vector expresses both GM-CSF and GFP from the EF1a promoter 
with an IRES sequence allowing us to use the expression of GFP as a marker to track GM-CSF expression. GM-CSF was stably over-expressed in ES-D3 cells by transfection. Flow cytometry analysis revealed that both GM-CSFexpressing and empty vector control ES-D3 cells express GFP at high levels in comparison to untransfected parental ES-D3 cells (Figure 2.2B). Once ES-D3 cells stably over-expressing GM-CSF were acquired, the amounts of GM-CSF secreted into the cell culture medium were determined using a murine GM-CSF ELISA kit. As shown in Figure 2.2C, the levels of GM-CSF generated by ES-D3 cells over-expressing GM-CSF were roughly equivalent to those produced by GM-CSF-expressing STO fibroblasts employed in an earlier study [29].

A

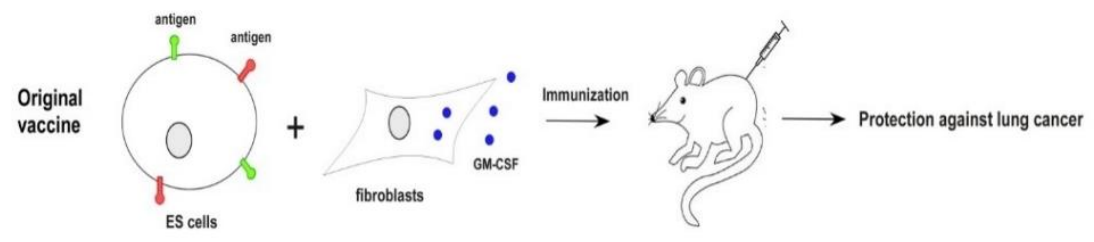

B

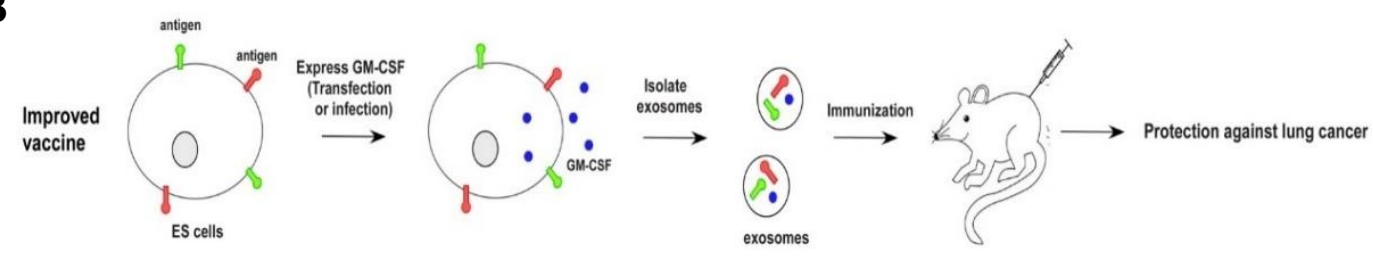

Figure 2.1. Strategies to generate vaccines against lung cancer.

(A) A previously reported vaccine comprised of allogeneic murine ESCs and murine fibroblasts expressing GM-CSF to promote immune responses. (B) An improved prophylactic vaccine comprised of exosomes derived from murine ESCs expressing GM-CSF. 
A

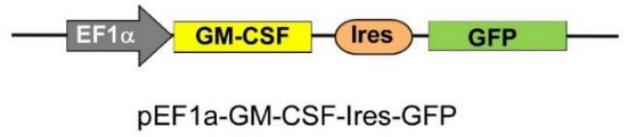

B

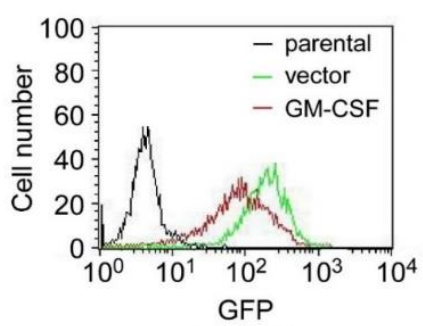

C

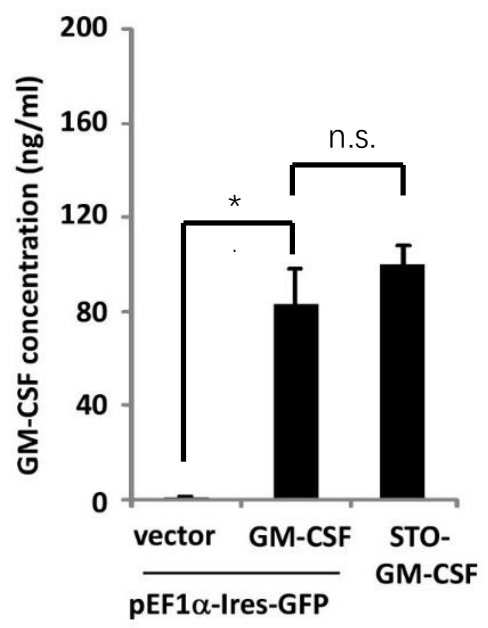

Figure 2.2. Exogenous GM-CSF was stably over-expressed in ES-D3 cells.

(A) In the schematic diagram of the plasmid for over-expressing murine GMCSF in ES-D3 cells, an EF1a promoter drives GM-CSF expression and GFP serves as an expression marker. (B) Fluorescence intensity of GFP in GMCSF-expressing ES-D3 cells or their empty vector control cells was determined by flow cytometry. (C) GM-CSF levels in transfected ES-D3 cells. ELISA measurements of GM-CSF concentrations in the medium of the indicated cells. The data are shown as mean \pm standard deviations (mean \pm SD) of three experiments, ${ }^{* *}, p<0.001$; n.s., not significant; ANOVA with Tukey's multiple comparison test. 


\section{Murine embryonic stem cells expressing GM-CSF maintain their pluripotency.}

As a cytokine with immunostimulatory function, GM-CSF has been reported to promote differentiation of hematopoietic progenitor cells [101]. To ensure that expressing GM-CSF in ES-D3 cells had limited effects on their pluripotent undifferentiated state, the pluripotency of ES-D3 cells was evaluated using flow cytometry to examine expression of multiple markers of pluripotency and differentiation. We assessed cellular expression of pluripotency-associated markers SSEA-1 and Oct-3/4 along with differentiation-associated marker SSEA-4. As shown in Figure 2.3, high expression levels of pluripotency markers SSEA-1 and Oct-3/4 were unchanged in GM-CSF-expressing cells compared with those in parental and vector control cells, and over $95 \%$ of each of parental and transfected ES-D3 cells were positive for the expression of SSEA-1 and Oct 3/4. Similarly, expression of the differentiation marker SSEA-4 remained at low levels in all of the cell lines examined with less than $1 \%$ of cells positive for SSEA-4 expression. Overall, these data provide evidence that over-expressing GM-CSF does not influence the pluripotency of murine ESCs. 

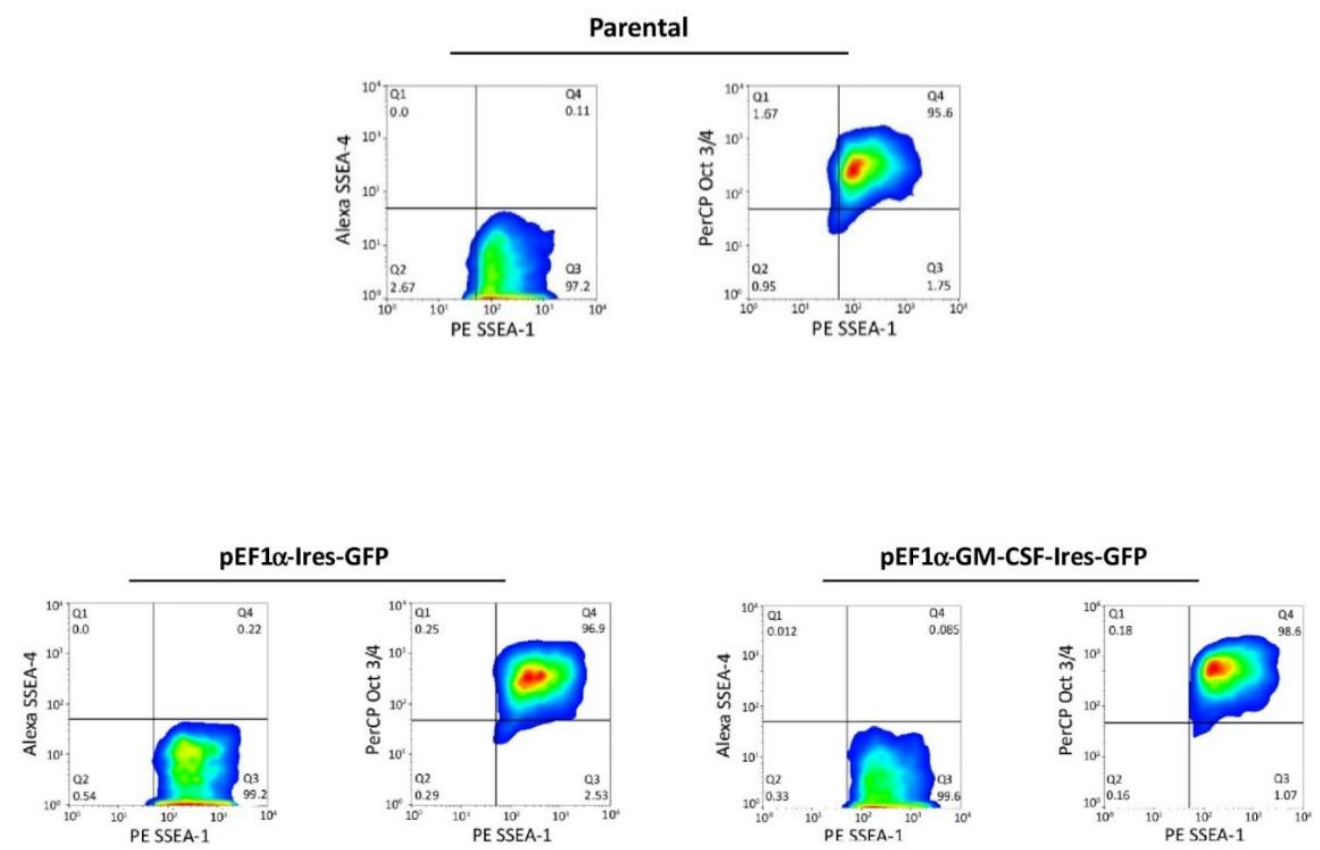

Figure 2.3. Pluripotency of ES-D3 cells is not affected by GM-CSF expression.

Flow cytometry analysis of the expression of pluripotency and differentiation markers (SSEA1, SSEA4 and Oct3/4) in parental, vector control and GMCSF-expressing ES-D3 cells. The data shown are representative of three independent flow cytometry assays. 


\section{Isolation and characterization of exosomes from murine embryonic stem cells.}

An increasing number of studies suggests that exosomes also exhibit immunoregulatory and anti-tumor functions [102]. Thus, we reasoned that exosomes from ES-DS cells expressing GM-CSF could also possess biological activities to regulate immune responses and prevent tumor growth. To this purpose, exosomes from ES-D3 cells stably expressing GM-CSF or its empty vector were isolated after several steps of centrifugation. Exosomes generated by vector control cells and GM-CSF-transfected cells were examined by TEM electron microscopy (Figure 2.4A). TEM images revealed that the exosomes were comprised of vesicles of variable sizes, a common feature of exosomal preparations [103]. Moreover, the diameters of individual vesicles were $30-100$ $\mathrm{nM}$, consistent with earlier reports [104]. Purity of exosomal/microvesicle material was indicated by positive western blot signals for different exosomal markers, including CD81, Alix and annexin V, flotillin-1 and CD86. Importantly, the abundance of exosomal markers was greatly enhanced in ES-D3-derived exosomes compared with corresponding whole cell extracts. In contrast, the presence of other subcellular compartment markers in exosomes was not detected, including (i) endoplasmic reticulum markers - protein disulfide isomerase (PDI) and calnexin, (ii) mitochondrial markers - cytochrome $\mathrm{C}$ and COX IV-subunit IV, and (iii) the cytosolic marker GAPDH, further demonstrating the purity of ES-D3-derived exosomal material (Figure 2.4B). To further 
characterize isolated exosomes from ES-D3 cells, we used a proteomics approach to analyze the profile of exosomal proteins. As expected, well-known exosomal protein markers, such as CD81, annexin $\mathrm{V}$ and Alix, were present in exosomes obtained from ES-D3 cells [Table 2.1].

A
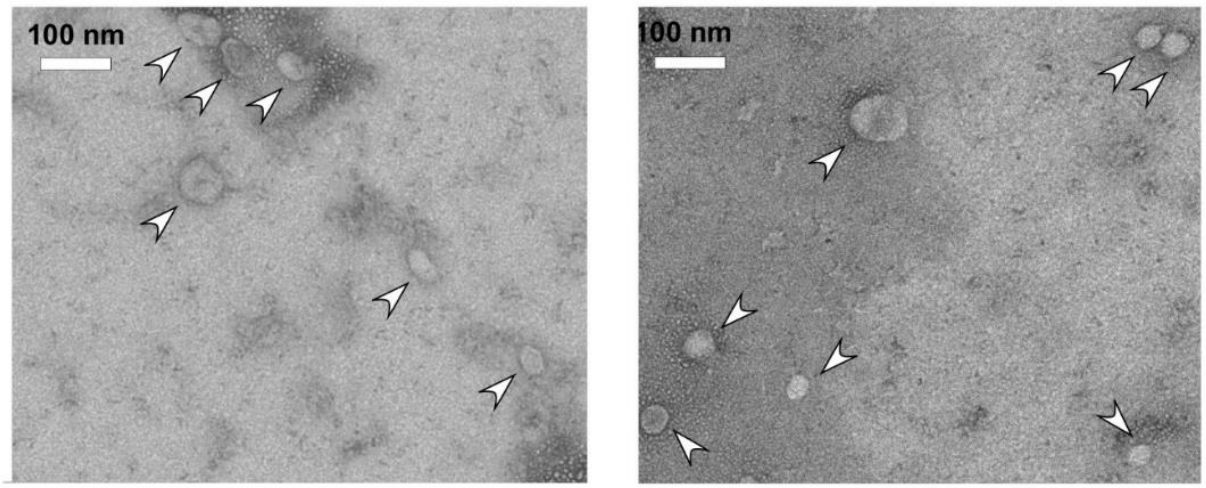

B

vector

GM-CSF
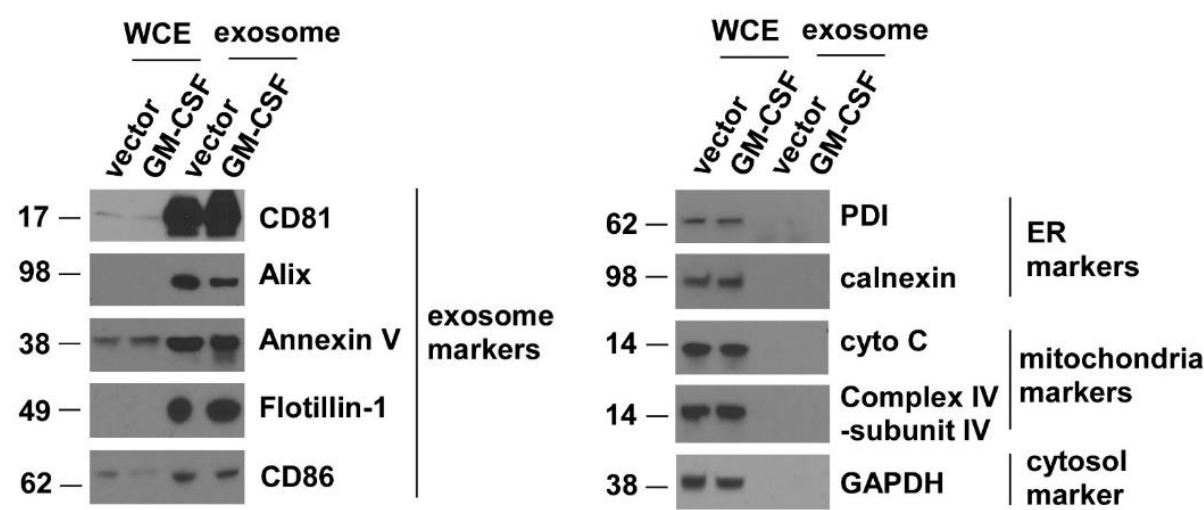

Figure 2.4. Characterization of exosomes isolated from ES-D3 cells.

(A) Exosomes were isolated from ES-D3 cells stably expressing GM-CSF or the empty vector. Exosomes were examined by transmission electron microscopy. Arrows indicate individual exosomes, scale bar $100 \mathrm{nM}$. (B) The levels of markers for exosomes, endoplasmic reticulum (ER), mitochondria, and cytosol in the indicated exosomes and whole cell extracts (WCE) were determined by western blot. PDI, protein disulfide isomerase; cyto $\mathrm{C}$, cytochrome C. Molecular weight markers (kD) are labeled on the left. 


\begin{tabular}{|c|c|c|}
\hline Category & Protein Name & Tumor Type \\
\hline Exosome marker & Programmed cell death 6-interacting protein/ Alix & N/A \\
\hline Exosome marker & Annexin II & N/A \\
\hline Exosome marker & Annexin V & N/A \\
\hline Exosome marker & CD9 & N/A \\
\hline Exosome marker & Transferrin receptor / CD71 & N/A \\
\hline Exosome marker & $\begin{array}{l}\text { Lysosome-associated membrane glycoprotein } 1 \\
\text { (LAMP 1) }\end{array}$ & N/A \\
\hline Exosome marker & Tumor susceptibility gene 101 (TSG101) & N/A \\
\hline Exosome marker & CD81 & N/A \\
\hline
\end{tabular}

Table 2.1. Exosomes derived from ES-D3 cells express prototype exosomal markers.

Exosomal markers in exosomes isolated from ES-D3 cells were identified by a proteomics approach. 


\section{The majority of GM-CSF is localized within exosomes.}

As shown earlier (Figure 2.2C), the majority of GM-CSF expressed in ES-D3 cells was extracellularly secreted. The concentrations of GM-CSF in isolated exosomes were investigated by ELISA. A significant amount of GM-CSF was detected in the exosomes isolated from ES-D3 cells over-expressing GM-CSF, but not in those prepared from vector control cells (Figure 2.5A). To further investigate GM-CSF protein localization in the exosomes, we quantitated exosomal GM-CSF levels under different experimental conditions through an ELISA-based approach (Figure 2.5B). Briefly, the detergent Tween-20 (0.05\%) was first employed to permeabilize the exosomal membranes, and ELISA assays were carried out in buffers with or without $0.05 \%$ Tween-20. Since Tween-20 is known to reduce protein-protein interactions, the background GMCSF levels detected in control exosomes were significantly reduced by Tween20 in the washing buffer. In contrast, the amounts of GM-CSF in the exosomes isolated from ES-D3 cells expressing GM-CSF were significantly increased by Tween-20. This result indicates that Tween-20 permeabilized exosomal membrane, making GM-CSF molecules located inside the exosomes available for detection. These data suggest that almost all GM-CSF is located in the lumen of the exosomes derived from ES-D3 cells. 
A

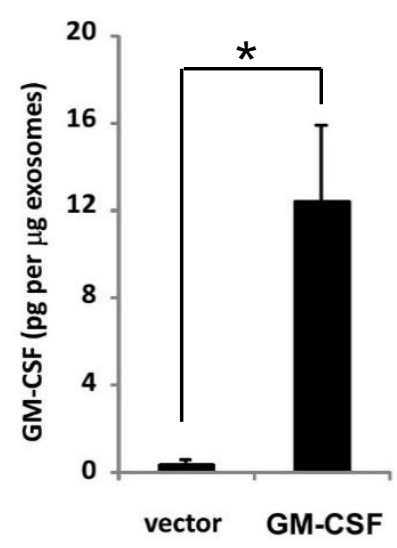

B

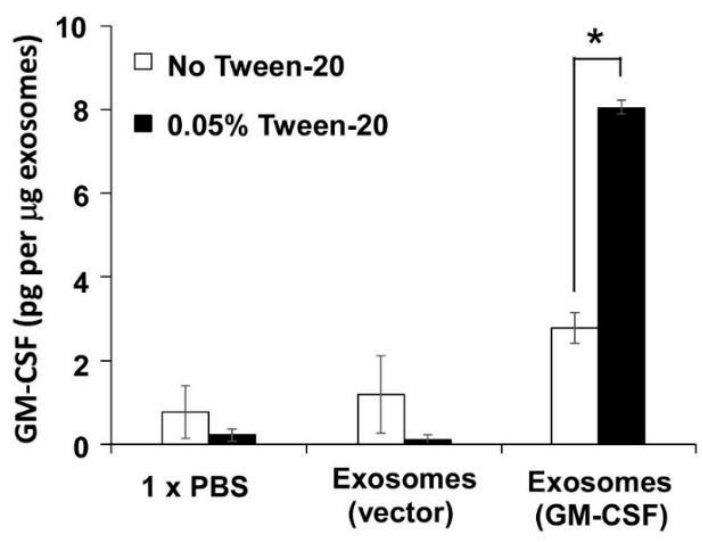

Figure 2.5. GM-CSF is located within the exosomes

(A) The amounts of GM-CSF in exosomes isolated from vector control ESD3 cells or GM-CSF-expressing ES-D3 cells were evaluated by ELISA. The data are presented as mean \pm SD of three independent experiments. *, $p<0.05$; ANOVA with Tukey's multiple comparison test. (B) The localization of GM-CSF in exosomes was examined. Exosomes pretreated with or without $0.05 \%$ Tween-20 were added to an ELISA plate. ELISA was carried out using washing buffer containing either 1 x PBS only or 1 x PBS $+0.05 \%$ Tween-20. The data are shown as mean \pm SD of three independent ELISA experiments. ${ }^{*}, p<0.05$; ANOVA with Tukey's multiple comparison test. 


\section{Vaccination with GM-CSF-expressing ESC-derived exosomes prevents the outgrowth of implanted lung adenocarcinoma.}

To explore the prophylactic potential of exosomes isolated from ESCs for lung cancer, a standard vaccination timing regimen was carried out (Figure 2.6A). Specifically, C57BL/6 mice $(n=20)$ were immunized twice (days 0 and 7$)$ with vehicle control, the exosomes of ES-D3 cells expressing empty vector (ES-exo) or the exosomes of GM-CSF-expressing ES-D3 cells (ES-exo/GM-CSF). Mice were then challenged with subcutaneous (s.c.) inoculation of Lewis lung carcinoma (LLC) cells at day 14 and monitored for tumor outgrowth. Robust protection against tumor initiation and growth was observed only in mice vaccinated with ES-exo/GM-CSF (Figure 2.6B). Vaccination of mice with ESexo/GM-CSF was $60 \%$ effective in preventing tumor outgrowth, whereas all non-vaccinated control animals developed tumors by day 28 post-challenge. More importantly, LLC tumors that developed in exo/GM-CSF-vaccinated mice $(n=8)$ were significantly smaller and exhibited greatly reduced tumor growth rate compared with non-vaccinated control and ES-exo-vaccinated mice (Figure 2.6C). Furthermore, vaccination with ES-exo was completely ineffective in reducing tumor outgrowth, revealing an essential role of immune-stimulatory GM-CSF for anti-tumor efficacy of the vaccine. 
A

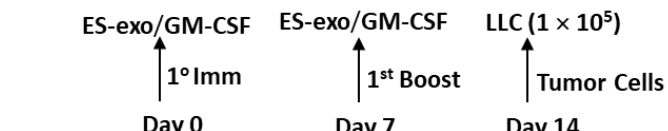
C57BL/6 Day 7 Day 14

B

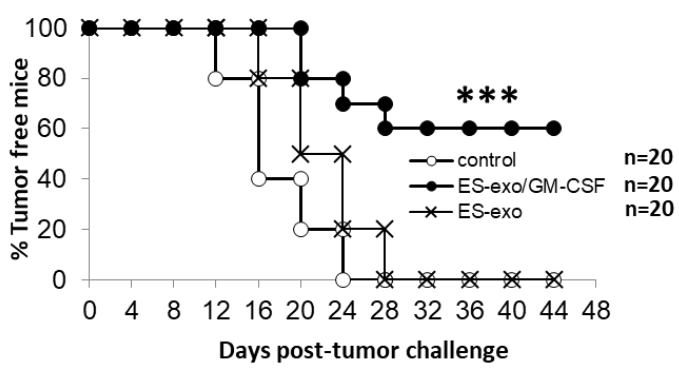

C

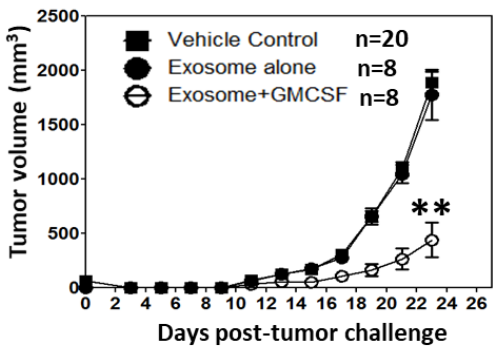

Figure 2.6. Vaccinating mice with the exosomes from ESCs expressing GM-CSF prevents implanted lung tumor growth.

(A) Scheme of immunization. Male C57BL/6 mice were immunized twice with $1 \times$ PBS (control), ES-exo or ES-exo/GM-CSF (Days 0 and 7) prior to s.c. challenge with LLC at Day 14. (B) Tumor growth was monitored daily in all animals (20 mice/group) until sacrifice due to tumors exceeding $5 \%$ of body weight. The ES-exo/GM-CSF-vaccinated tumor-free mice remained so for up to 4 months later with no overt signs of distress. Results are representative of three independent experiments. ${ }^{* * *}, p<0.0001$; relative to control group; log-rank test. (C) Tumor growth was measured with calipers every 2 nd or 3 rd day and tumor volumes were plotted as indicated. The data are represented as mean \pm SD the average of tumor volumes of (with 20 mice/ in control group, 8 mice in ES-exo group and 8 mice/in ES-exo/GMCSF group and are representative of three independent experiments. ${ }^{* *}, p<$ 0.001; ANOVA with Tukey's multiple comparison test. 


\section{Vaccination with ES-exo/GM-CSF has no toxic side effects in mice.}

To evaluate whether any toxic effects are associated with the regimen of two weekly vaccinations, we examined fertility, kidney function, liver function, and complete blood count $(\mathrm{CBC})$ in vaccinated and unvaccinated mice. For fertility, there was no difference in litter sizes between female animals vaccinated with ES-exo/GM-CSF and their control counterparts (Figure 2.7A). Moreover, vaccination with ES-exo/GM-CSF did not alter the gender ratio of litters (Figure 2.7B).

As shown in Figures 2.8A-B, the levels of alanine transaminase (ALT) and alkaline phosphatase (ALP) were similar among control, ES-exo and ESexo/GM-CSF-vaccinated mice, indicating that liver function remained unchanged after vaccination. To assess kidney function, we measured the levels of creatinine and blood urea nitrogen (BUN) in serum, and slight changes in BUN and creatinine levels between the vaccinated group and control group were detected (Figures 2.8C-D). Finally, complete blood count (CBC) analysis were carried out (Figures 2.9), showing no significant difference in blood cell counts among the mice in different experimental groups. Overall, these studies indicate that vaccination with ES-exo/GM-CSF did not induce any toxic effects in the immunized mice. 
A

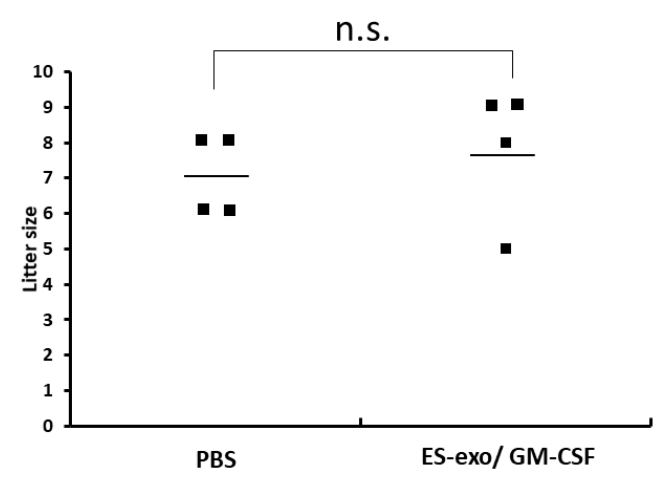

B

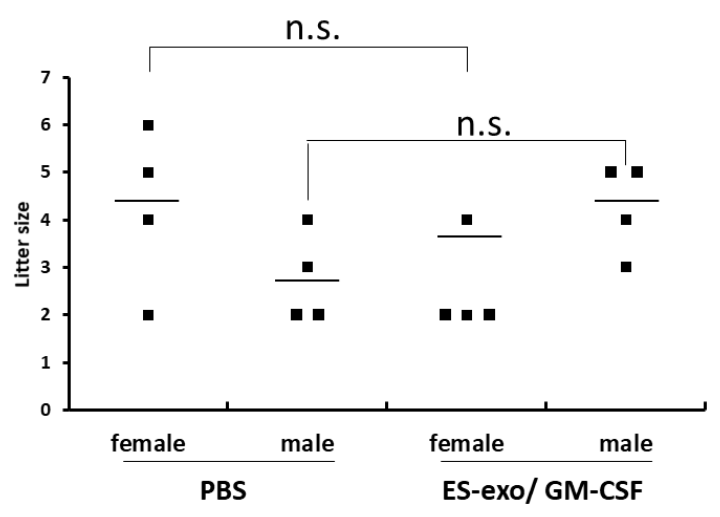

Figure 2.7. Vaccination with ESC-derived exosomes (ES-exo/GM-CSF) does not reduce fertility of mice.

Following immunization twice with vehicle control (1 x PBS) or ES-exo/GMCSF (days 0 and 7), female C57BL/6 mice were bred with C57BL/6 male mice at Day 14. (A)The dot graph showing the litter sizes of unvaccinated control or ES-exo/GM-CSF-vaccinate female mice (4 mice in each group) on the date of delivery. The average values of litter sizes are also presented. n.s., not significant, ANOVA with Tukey's multiple comparison test. (B)The numbers of two genders in each litter 4 weeks following birth. The mean values of the numbers of newly born male or female mice is shown $(n=4$ per group, n.s., not significant; ANOVA with Tukey's multiple comparison test). 
A
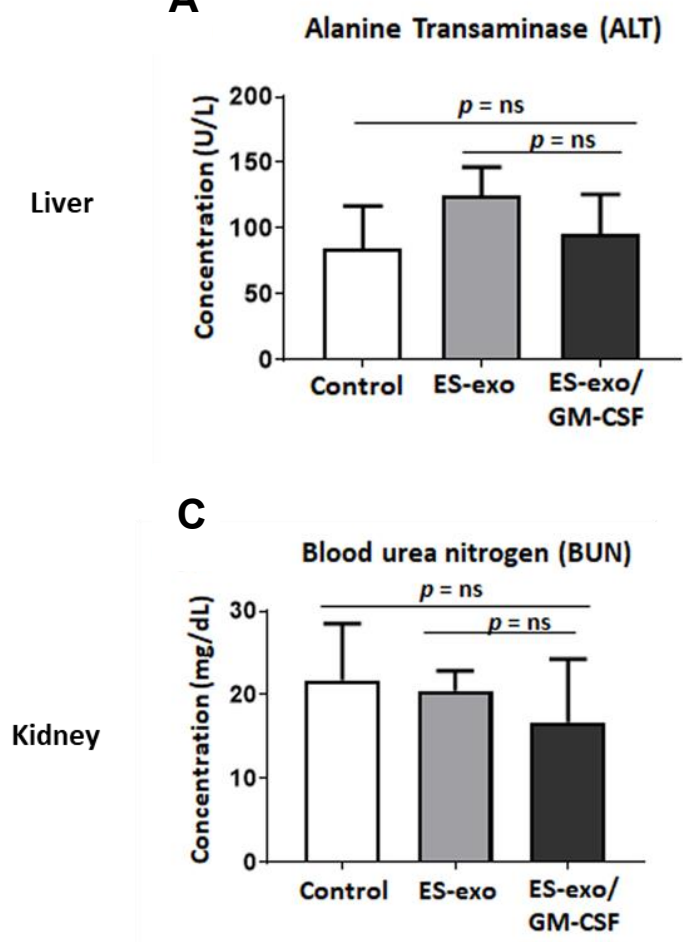

B

Alkaline Phosphatase (ALP)

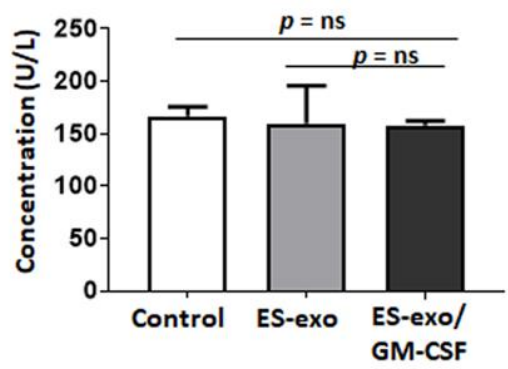

D

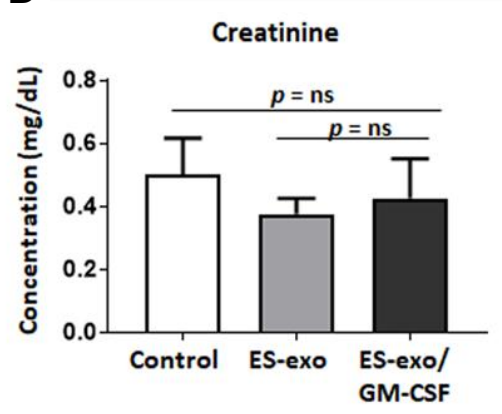


Figure 2.8. ES-exo/GM-CSF vaccination does not display toxic effects on functions of kidney and liver.

Male C57BL/6 mice were vaccinated twice with vehicle only (control), ESexo or ES-exo/GM-CSF (days 0 and 7 ). The serum was collected from each mouse and functions of liver and kidney were evaluated at Day 14. (A-B) Liver function was determined by examining the levels of alanine aminotransferase (ALT) and alkaline phosphatase (ALP). Bar graph showing ALT and ALP levels in serum obtained from mice vaccinated with vehicle only (control), ES-exo or ES-exo/GM-CSF. (C-D) Kidney damage was assessed by measuring the levels of blood urea nitrogen (BUN) and creatinine. Bar graph showing the levels of BUN and creatinine in serum acquired from the indicated mice. All of the data are presented as mean \pm SD of measured values with 4 mice in each group. n.s., not significant, ANOVA with Tukey's multiple comparison test. 


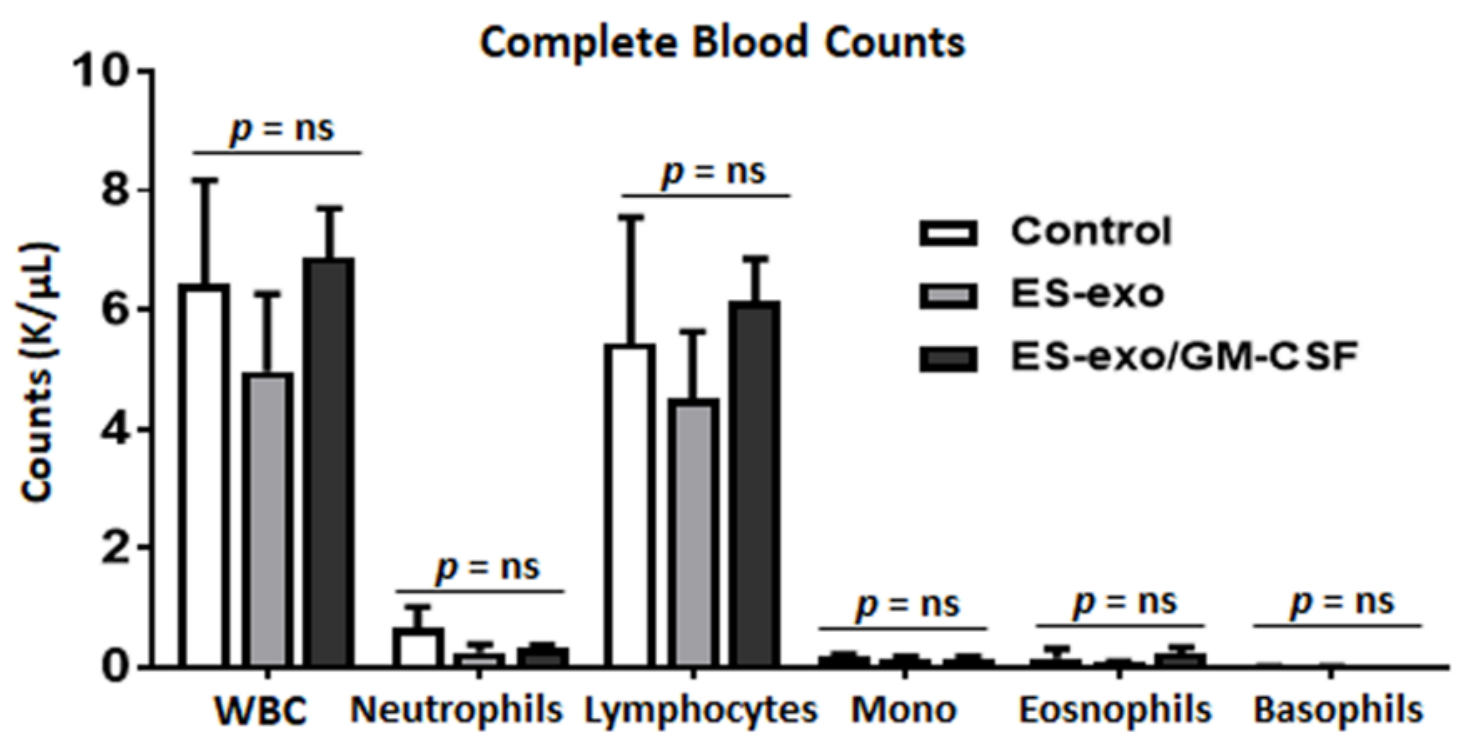

Figure 2.9. ES-exo/GM-CSF vaccination has no influence on blood cell counts.

Male C57BL/6 mice were immunized twice with vehicle only (control), ESexo or ES-exo/GM-CSF (days 0 and 7). At day 14, whole blood was collected from each mouse and examined by complete blood count (CBC). Bar graph showing the levels of different cell types in blood obtained from the indicated mice. The data represent mean \pm SD of cell numbers. $(n=4$ per group, mean \pm SD; n.s., not significant, ANOVA with Tukey's multiple comparison test). 


\section{Vaccination with ES-exo/GM-CSF induces tumor cell-specific Th1- mediated cytokine response in $\mathrm{CD}^{+} \mathrm{T}$ cells.}

We next determined the ability of $\mathrm{CD} 8^{+} \mathrm{T}$ cells from vaccinated mice to produce effector cytokines required for effective anti-tumoral cytolytic activity. These experiments were carried out by the laboratory of Dr. Kavitha Yaddanapudi. In response to re-stimulation with LLC cell lysate, a significantly higher frequency of IFN- $\gamma$ - and TNF- $\alpha$-producing $\mathrm{CD}^{+}$splenocytes were obtained from ESexo/GM-CSF-vaccinated mice when compared with the non-vaccinated control and ES-exo-vaccinated mice (Figure 2.10). Importantly, analysis of the phenotype of tumor-infiltrating immune cells supports the concept that vaccination led to an immune-based suppression of tumor growth.

Using tumors isolated from unvaccinated, ES-exo- and ES-exo/GM-CSFvaccinated mice, we found substantial increases in IFN- $\gamma$ - and TNF- $\alpha-$ producing $\mathrm{CD}^{+} \mathrm{T}$ cells only in mice vaccinated with exosomes prepared from GM-CSF-expressing ESCs in response to re-stimulation with LLC lysate (Figure 2.11). 
A

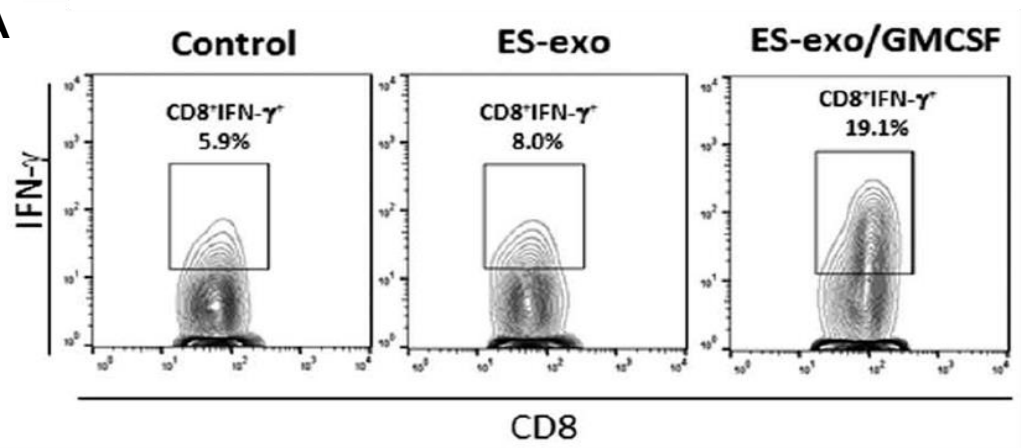

B

C
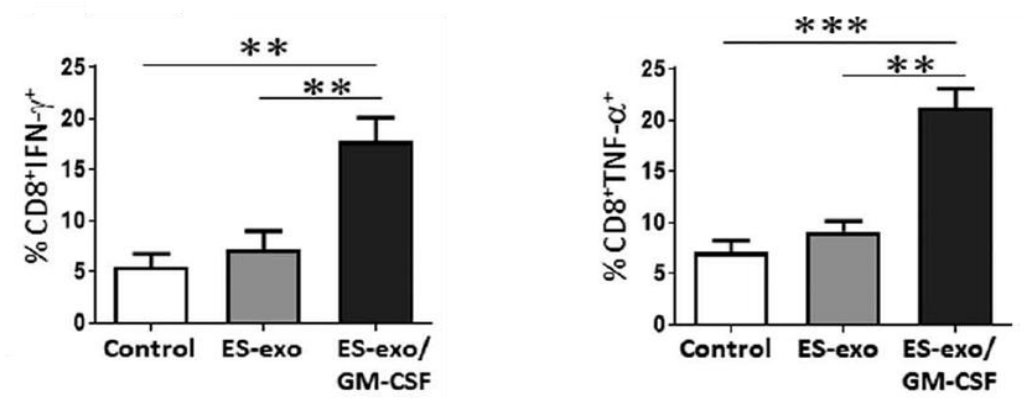

Figure 2.10. ESC-derived exosome vaccination induces Th1-mediated cytokine responses in splenic $\mathrm{CD8}^{+} \mathrm{T}$ cells.

C57BL/6 mice were immunized twice (days 0 and 7 ) with vehicle only (control, 1 x PBS), ES-exo or ES-exo/GM-CSF. Ten days after the boost, splenocytes from mice were stimulated with LLC lysate and surface expression of CD3, CD8 and intracellular expression of cytokines and analyzed by flow cytometry. (A) Dot plots showing IFN- $\gamma$ expression in $\mathrm{CD}^{+}$ T cells in splenocyte cultures obtained from the indicated mice. Numbers in graphs represent the percentages of each subpopulation. $(\mathbf{B}, \mathbf{C})$ Bar graphs showing percentages of $\mathrm{CD}^{+} \mathrm{IFN}-\gamma^{+}$, and $\mathrm{CD} 8^{+} \mathrm{TNF}-\alpha^{+}$in splenocyte cultures derived from control, ES-exo- or ES-exo/GM-CSF-vaccinated mice. Results are expressed as percentages of total cells ( $n=6$ per group, mean $\pm S D,{ }^{* *}, p<0.001 ;{ }^{* * *}, p<0.0001 ;$ ANOVA with Tukey's multiple comparison test). 
A

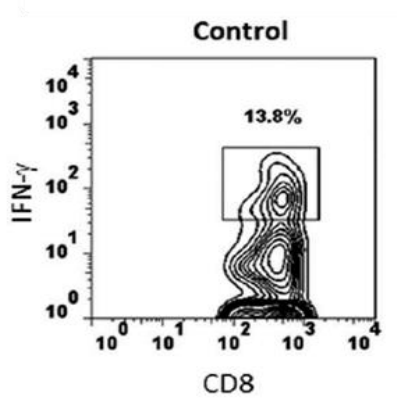

B

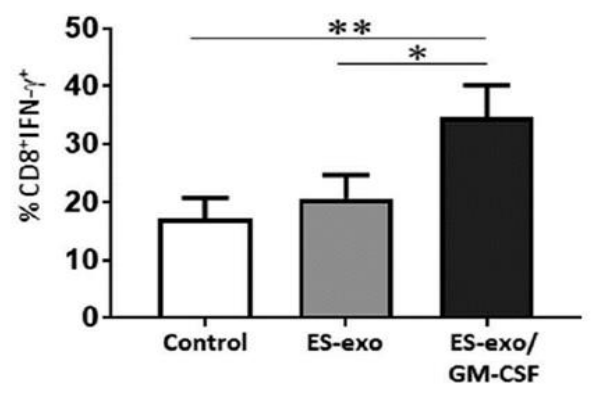

ES-exo

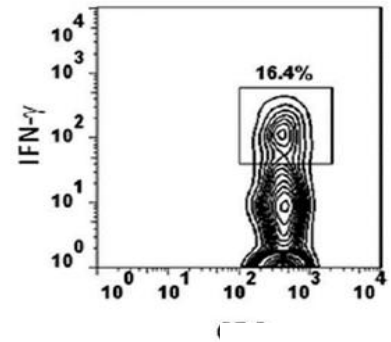

C
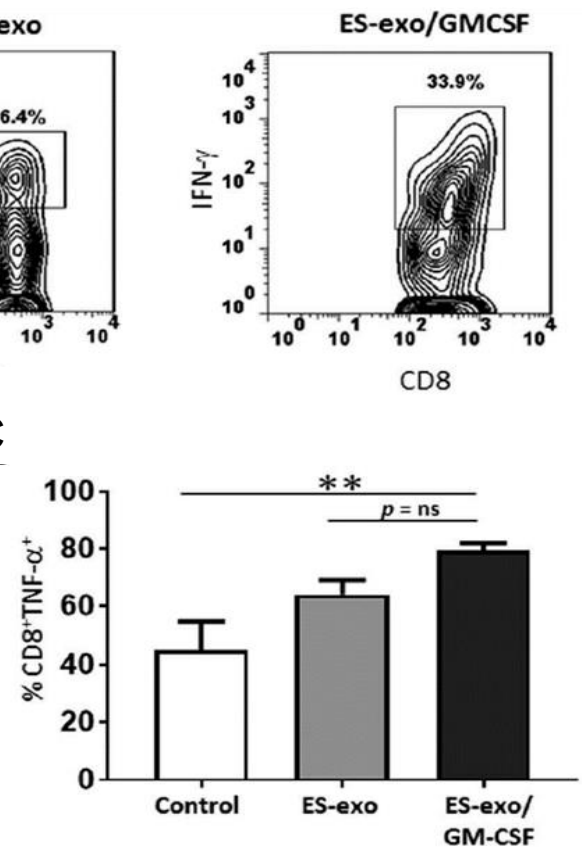
Figure 2.11. Vaccination with ESC-derived exosomes promotes Th1mediated cytokine responses in intratumoral $\mathrm{CD}^{+} \mathrm{T}$ cells.

Following immunization with vehicle only (control), ES-exo or ES-exo/GMCSF twice (days 0 and 7), C57BL/6 mice were challenged with injection of LLC (s.c.) on day 14. Tumor-infiltrating cells were collected $15-18$ days later and stimulated with LLC lysate $(50 \mathrm{mg} / \mathrm{ml})$ for 24 hours. Cells were then restimulated for 6 hours with LLC lysate $(50 \mathrm{mg} / \mathrm{ml})$ in the presence of Brefeldin $\mathrm{A}(1 \mu \mathrm{L} / \mathrm{ml})$. Surface expression of CD8 and intracellular expression of cytokines were evaluated by flow cytometry. The pan-hematopoietic marker CD45 was used to identify intratumoral immune cells. (A) Dot plots showing IFN- $\gamma$ expression in $\mathrm{CD} 45^{+} \mathrm{CD} 3^{+} \mathrm{CD} 8^{+}$cells obtained from control, ES-exo- or ES-exo/GM-CSF-vaccinated mice. Numbers in graphs represent the percentages of each subpopulation. (B, C) Bar graphs showing percentages of $\mathrm{CD} 45^{+} \mathrm{CD}^{+} \mathrm{CD} 8^{+} \mathrm{IFN}-\gamma^{+}(\mathrm{B})$ and $\mathrm{CD} 45^{+} \mathrm{CD} 3^{+} \mathrm{CD} 8^{+} \mathrm{TNF}-\mathrm{a}^{+}$ (C) in tumors derived from the indicated mice. Results are demonstrated as percentages of IFN- $\gamma^{+}$cells or TNF- $\alpha^{+}$cells in $\mathrm{CD} 45^{+} \mathrm{CD} 3^{+} \mathrm{CD} 8^{+}$cells $(n=6$ per group, mean $\pm S D,{ }^{*}, p<0.05^{* *}, p<0.001 ;$ n.s., not significant, ANOVA with Tukey's multiple comparison test). 


\section{Vaccination with GMCSF-expressing ESC-derived exosomes increases the ratio of $\mathrm{CD8}^{+} \mathrm{T}$ effector cells to $\mathrm{T}_{\text {regs }}$ in the tumor.}

Our results so far suggest that ES-exo/GM-CSF vaccine-induced antitumor efficacy is reliant on $\mathrm{CD}^{+} \mathrm{T}$ effector cells. Therefore, Dr. Kavitha Yaddanapudi's group analyzed effects of our vaccination strategy on the phenotype of tumor-infiltrating $\mathrm{CD} 8^{+} \mathrm{T}$ cells and Tregs, a prominent suppressor subset that hamper anti-tumoral effector responses. Tumors from controls and vaccinated mice (from the small numbers of ES-exo/GM-CSF-vaccinated mice that did develop LLC lesions) were harvested and used to investigate the subset profiles of tumor-infiltrating immune cells. Flow cytometry analysis showed a significant decrease in the percentage of $\mathrm{CD} 4^{+} \mathrm{CD} 25^{+} \mathrm{Foxp} 3^{+} \mathrm{T}_{\text {regs }}$ in tumor infiltrates from ES-exo/GM-CSF-vaccinated mice when compared with non-vaccinated control and ES-exo-vaccinated mice (Figures 2.12A-B) and the ratio of $C D 8^{+} T$ cells to $T_{\text {regs }}$ was significantly increased in the tumor infiltrates from ES-exo/GM-CSF-vaccinated mice (Figure 2.12C). Additionally, CD8 ${ }^{+}$cells in the ES-exo/GM-CSF tumor infiltrates had significantly elevated expression of the activation marker CD25 (Figure 2.12D). 
A
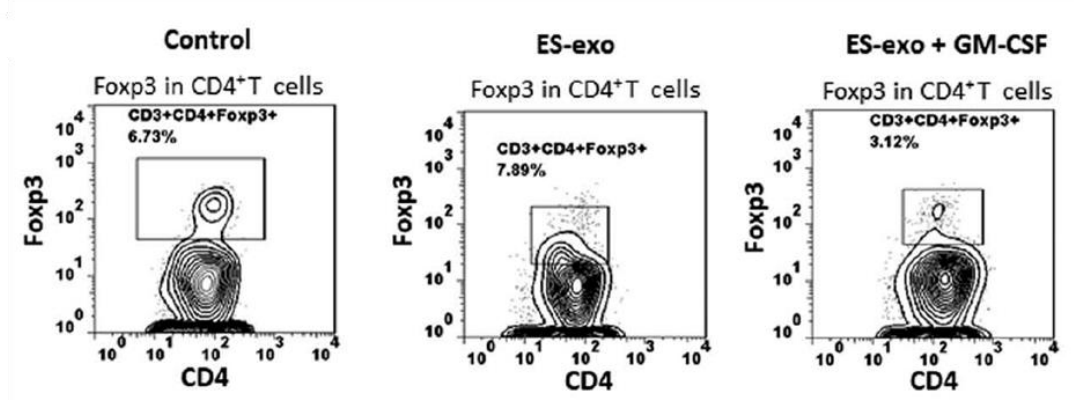

B

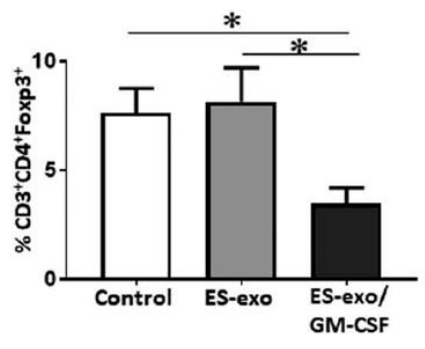

C

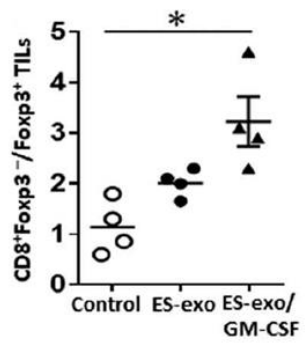

D

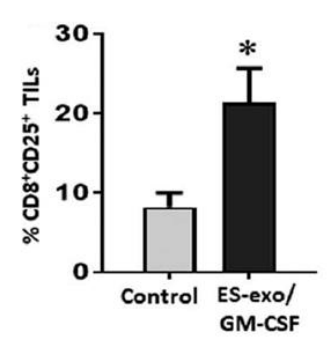


Figure 2.12. ES-exo/GM-CSF vaccination decreases $T$ regulatory ( $\left.T_{\text {regs }}\right)$ cells and increases the ratio of effector $\mathrm{CD8}^{+} \mathrm{T}$ cells to $\mathrm{T}_{\text {regs }}$ in the tumors.

C57BL/6 mice were vaccinated twice (days 0 and 7) with vehicle only (control), ES-exo or ES-exo/GM-CSF followed by s.c. inoculation of LLC on day 14 . Tumor-infiltrating cells were analyzed by flow cytometry $15-18$ days later using the pan-hematopoietic marker CD45 to distinguish tumorinfiltrating immune cells from tumor cells. (A) Dot plots showing the percentages of Foxp3 ${ }^{+}$Tregs in tumor-infiltrating $\mathrm{CD}^{+}{ }^{+} \mathrm{CD} 4^{+}$cells obtained from the indicated mice. Numbers in graphs are the percentages of each subpopulation. (B) Bar graphs showing the percentages of Foxp3 ${ }^{+} \mathrm{T}_{\text {regs }}$ subpopulations in tumor-infiltrating $\mathrm{CD} 3^{+} \mathrm{CD} 4^{+}$cells $(\mathrm{n}=4$ per group, mean \pm SD, *, p<0.05; ANOVA with Tukey's multiple comparison test). (C) Dot graph showing the ratio of $\mathrm{CD}^{+}{ }^{+} \mathrm{Foxp} 3^{-}$to $\mathrm{CD} 8{ }^{-}{ }^{-}{ }$oxp $3^{+}$cells in 1 of 2 representative experiments $\left(n=4\right.$ per group, mean $\pm S D,{ }^{*}, p<0.05$; ANOVA with Tukey's multiple comparison test). (D) ESC-derived exosome vaccination increases the frequency of functional $C D 8^{+} \mathrm{T}$ cells in tumors. Bar graph showing the percentages of $\mathrm{CD} 25^{+} \mathrm{CD} 8^{+}$in $\mathrm{CD} 45^{+}$tumor-infiltrating cells obtained from control and ES-exo/GM-CSF-vaccinated mice. Results are expressed as percentages of $\mathrm{CD} 45^{+}$cells. The data represent results from 2 independent experiments with 3 mice/group. ${ }^{*}, p<0.05$; relative to control group; unpaired $\mathrm{t}$ test. Error bars represent mean $\pm \mathrm{SD}$. 


\section{ESC-derived exosome vaccination prevents the outgrowth of an implanted mammary carcinoma.}

To test the effectiveness of ES-exo/GM-CSF vaccine in targeting multiple cancer types, an additional experiment was performed using the mammary carcinoma cell line $4 T 1$, which is syngeneic to the Balb/c mouse strain. Female Balb/c mice were divided into vehicle control, ES-exo and ES-exo/GM-CSF groups (10 per group) and vaccinated for two weeks (s.c. route). Following vaccination, $4 \mathrm{~T} 1$ cells $\left(1 \times 10^{5}\right)$ were subcutaneously injected (Figure $\left.2.13 \mathrm{~A}\right)$. Tumor growth assessment by caliper measurement showed significantly lower tumor progression in the ES-exo/GM-CSF group (Figure 2.13B). Using splenocytes isolated from unvaccinated, ES-exo- and ES-exo/GM-CSFvaccinated mice, we found a significant increases in IFN- $\gamma$-producing $C D 8^{+} T$ cells in mice vaccinated with ES-exo/GM-CSF in response to re-stimulation with 4T1 lysate (Figure 2.13C, D).

A

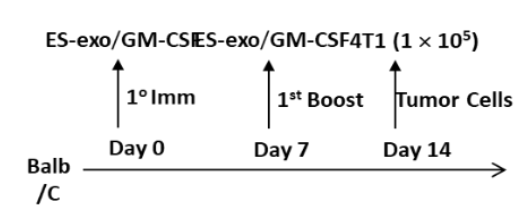

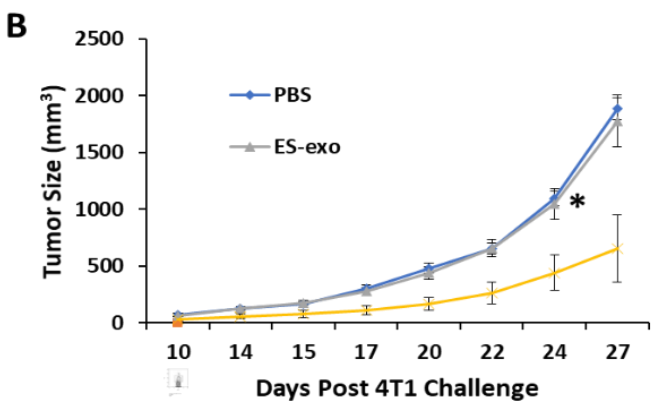

C

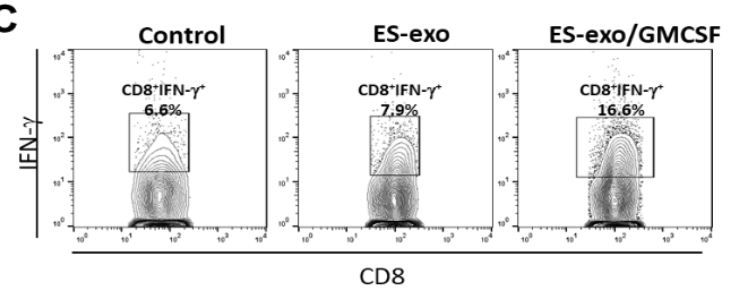

D

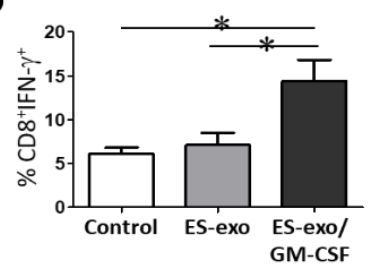


Figure 2.13. Vaccination with ES-exo/GM-CSF slows the outgrowth of an implanted mammary carcinoma.

(A) Scheme of immunization. Female Balb/c mice were immunized twice (days 0 and 7) with vehicle only (control), ES-exo or ES-exo/GM-CSF before s.c. challenge with syngeneic $4 \mathrm{~T} 1$ cells on day 14 . (B) Tumor growth was monitored by measuring tumor volumes. The data represent the average tumor volumes of 20 mice/group. Error bars represent mean \pm SD. (C, D) ESC-derived exosome vaccination induces Th1-mediated cytokine responses in splenic $\mathrm{CD} 8^{+} \mathrm{T}$ cells. Splenocytes from vaccinated and control mice were co-cultured with $4 \mathrm{~T} 1$ lysate $(50 \mathrm{mg} / \mathrm{ml})$ for 4 days before restimulation for 6 hours with $4 \mathrm{~T} 1$ lysate $(50 \mathrm{mg} / \mathrm{ml})$ in the presence of Brefeldin A $(1 \mu \mathrm{l} / \mathrm{ml})$. After restimulation, surface expression of CD8 and intracellular expression of IFN-g were evaluated by flow cytometry. (C) Dot plots showing IFN- $\gamma$ expression in $\mathrm{CD}^{+}$cells in splenocyte cultures obtained from the indicated mice. Numbers in graphs represent the percentages of each subpopulation. (D) The data shown in (C) are summarized. Results are presented as percentages of IFN- $\gamma$-positive cells in $\mathrm{CD}^{+}$cells $\left(n=4\right.$ per group, mean $\pm S D,{ }^{*}, p<0.05$; ANOVA with Tukey's multiple comparison test). 


\section{DISCUSSION}

Traditionally, among different approaches of treatment, vaccines are a very safe and effective strategy to prevent infectious diseases. However, the utilization of vaccination in cancer prevention is limited. The only verified prophylactic anticancer vaccination in clinics is the vaccine against human papilloma virus (HPV) that causes the greatest risk for cervical cancer [105]. This is a conventional vaccine based on hollow virus-like particles. Unfortunately, most types of cancers are not induced by infection. The development of prophylactic vaccine against non-infection related cancers is still at early stages.

Fetal tissues have been reported to prevent transplantable tumors in the $20^{\text {th }}$ century [18]. In a previous study [29], ESCs along with a source of GMCSF have been shown to successfully block the outgrowth of implanted as well as carcinogen-induced lung tumors in mice. To eliminate the risk of teratoma formation induced by ESCs, we generated ESCs over-expressing GM-CSF and isolated the exosomes from the cells as a vaccine against implanted lung tumors in the present study (Figure 2.1). As the effectiveness of embryonic materials in tumor prevention is likely to be attributed to their pluripotent characteristics shared with cancer cells, we ensured that exogenous expression of GM-CSF did not influence the pluripotency of the transfected ESD3 cells (Figure 2.3). Furthermore, the exosomes isolated from the ES-D3 cells appear to possess high purity without detectable contamination from other cellular constituents, such as cytoplasm and organelles (Figure 2.4). 
As a monomeric glycoprotein cytokine, GM-CSF functions to stimulate the development of the immune system, such as activation of immune/inflammatory cascades [37]. Earlier studies indicate that GM-CSF by itself lack cancer prevention efficacy. In prophylactic setting, an ES cell vaccine composed of irradiated allogeneic murine ESCs and GM-CSF-expressing murine STO fibroblasts was very effective in preventing lung malignancies [29]. Interestingly, STO fibroblasts expressing GM-CSF failed to provide any protection against the outgrowth of LLC tumors, suggesting that the observed protection with this vaccine is not due to non-specific immune responses evoked by GM-CSF [29]. Therefore, it is unlikely that GM-CSF molecules in the exosomes in ES-exo/GM-SCF vaccine are solely responsible for anti-tumor immunity.

In our research, exosomes isolated from ESCs expressing GM-CSF could be used as a vaccine for the prevention of transplanted lung tumor outgrowth compared with the exosomes isolated from vector control cells (Figure 2.6). In those animals that did develop tumors, tumor growth was significantly slower when compared to unvaccinated control animals and those vaccinated with the exosomes from ESCs lacking GM-CSF expression. In the latter group of animals, tumor outgrowth did not differ from that detected in unvaccinated mice. These findings indicate that GM-CSF is a potent stimulator of immune responses during vaccination, which is in agreement with earlier reports [29, 106]. Indeed, the immunostimulatory property of GM-CSF has been recently 
exploited by Bencherif et al. for amplification of immune responses in a melanoma model [107].

In our experimental tumor model, ES-exo/GM-CSF combination vaccine significantly increases the ratio of $\mathrm{CD}^{+} \mathrm{T}$ cells to $\mathrm{T}_{\text {regs }}$ and the percentages of $\mathrm{CD}^{+} \mathrm{CD}^{2} 5^{+}$and $\mathrm{CD} 8^{+} \mathrm{IFN}-\gamma^{+}$effector cells within the tumors, suggesting effective vaccine-induced, tumor-reactive immune system priming. These immunophenotyping data lend additional support to the tentative conclusion that such vaccination might be a viable approach to the prevention of cancers in humans. Interestingly, the majority of detected GM-CSF was located inside exosomes (Figure 2.5). Therefore, as an effective immunogen, the immunologic activation by GM-CSF likely arises from exosomal fusion with the plasma membrane of antigen-presenting cells.

The underlying mechanisms of the cancer-preventive efficacy of ESexo/GM-CSF vaccination are not fully understood. It is highly likely that exosome-based vaccine elicits similar immune responses as its intact cellbased counterpart [29]. Anti-tumor immunity evoked by ES-exo/GM-CSF vaccination may be attributed to an increase in an effective repertoire of $\mathrm{CD}^{+}$ cytotoxic $\mathrm{T}$ cells, enhanced infiltration of $\mathrm{CD}^{+} \mathrm{T}$ cells into the tumor microenvironment, and decreased tumor suppressive $\mathrm{T}_{\text {regs }}$ in tumors.

Extracellular vesicles (EVs) range in size from $20 \mathrm{~nm}$ to $200 \mathrm{~nm}$ in diameter [55]. Exosomes, a subtype of EVs, are membrane vesicles with variable size of 30-100 nm in diameter. In Figure 2.4A, TEM images revealed that the sizes of 
vesicles in our samples were consistent with those of exosomes reported previously. Purity of exosomal preparations was further demonstrated by western blot analysis (Figure 2.4B), indicating the predominant presence of exosomes in our samples. Due to overlapping molecular markers between exosomes and other types of EVs, we could not completely exclude the possibility of small amounts of EVs other than exosomes existing in our preparations [90]. Additional experimental approaches, such as immunogold TEM staining [108] and nanoparticle tracking analysis [109], could be utilized to further characterize exosomal preparations from ESCs.

Exosomes, initially defined as cell-derived nanovesicles, were first described in 1946 when they were isolated from platelets [56]. Recently, evidence has emerged that exosomes could stimulate immune responses in cancer treatment [87]. The exosome-based anti-cancer therapies have high stability, in vivo bioavailability, and an inherent ability to stimulate anti-tumor immune responses. Exosomes do not endogenously replicate, as whole cells do, and exosomes can be readily bioengineered to a clinical grade and scaled up for dosing requirements [91]. The idea of using tumor-derived exosomes as a vaccine has been proposed earlier [110], although this approach is complicated by the fact that such preparations may also be strongly immunosuppressive. Furthermore, tumor cells expressing GM-CSF have been explored to serve as a therapeutic vaccine with variable success in certain animal models [111, 112]. In pre-clinical studies, exosomes obtained from 
matured dendritic cells (DCs) express more abundant MHC-I and MHC-II molecules as well as co-stimulatory molecules (e.g., CD40, CD80, and CD86) and induce potent antigen-specific anti-tumor $\mathrm{T}$ effector responses shown by cytotoxic T lymphocytes (CTLs) and natural killer (NK) cells both in vitro and in vivo $[71,113,114]$. Similarly, the exosomes isolated from ESCs comprises stable vesicles harboring protein contents that can be tailor-manufactured from human cell lines in clinical grade (cGMP) quality [91]. Furthermore, ES-derived exosomes can be produced in large quantities and cryo-preserved for more than 6 months at $-80^{\circ} \mathrm{C}$ with their functional activity intact [103].

As a promising biological agent to prevent the outgrowth of lung cancer, exosomes from ES-D3 cells expressing GM-CSF need to exhibit limited toxic effects associated with the vaccination. Usually, the side effects of a biological immune modulator or biological agent are different from conventional drugs [115], and they are always linked to the biological effects induced by the agent. There are five types of reactions of the adverse side-effects from biological agents, including cytokine related syndrome, immediate and delayed hypersensitivity reactions, immune imbalance syndrome, cross-reactions on different tissues and non-immunological side-effects [116]. Particularly, these reactions can be partially reflected from biological evaluations, such as $\mathrm{CBC}$, liver and kidney functions. Indeed, we did not observe any significant changes in biological functional parameters between vaccinated and unvaccinated animals, indicating that ES-exo/GM-CSF is a safe vaccination strategy without 
detectable side effects (Figure 2.7-9).

In our studies, ES-exo/GM-CSF immunization elicited immunologic rejection of transplantable tumors (Figure 2.6). This raised a concern that the vaccination strategy might have deleterious effects on fertility of vaccinated female mice, as embryos could be eliminated by the immunity generation by vaccine. As shown in Figure 2.7, ES-exo/GM-CSF vaccine exerted little influence on the fertility of female mice. This could be explained by the difference in antigen repertoires between mouse embryos and exosomes from ESCs. As a result, vaccination with ES-exo/GM-CSF confers limited crossimmunity to embryos. In addition, maternal immune tolerance, the immune tolerance towards the semi-allogeneic conceptus during pregnancy, is also involved in protecting embryos from being rejected. Emerging evidence indicate that a number of immunosuppressive factors produced by placenta are crucial for maternal-fetal tolerance. Among them, placenta-derived exosomes bearing human ligands of activating NK cell receptor NKG2D have been found to promote the fetal immune escape by decreasing the cognate receptor expression in a variety of immune cells [117].

The effects of aging on the immune system are well documented, as the age of patients has great impacts on the immune responses and the outcomes of treatment against lung cancer $[118,119]$. Consequently, elderly lung cancer patients generally do not respond to immunotherapy as well as younger patients. In our study, we investigated the cancer prevention efficacy of 
exosome-based vaccine in mice at young age. In the future, animals with different ages will be studied to evaluate how aging might influence the vaccination efficacy of ES-exo-GM-CSF.

Finally, a recent publication shows that human and murine induced pluripotent stem cells (iPSCs) express tumor-associated antigens, and that irradiated autologous iPSCs, in a prophylactic setting, prevent tumor growth in syngeneic murine breast cancer, mesothelioma, and melanoma models [30]. This study provides further support to our vaccination strategy utilizing exosomes from GM-CSF-expressing ES-D3 cells as an effective immunepreventive lung cancer vaccine. 
CHAPTER III

\section{MURINE ESC-DERIVED EXOSOMES FUNCTION AS A CELL-FREE} VACCINE AGAINST METASTASIZED LUNG CANCER

\section{INTRODUCTION}

It is well established that the immune system plays an essential role in lung cancer therapy. Lung cancer cells are known to elicit suppressive immune response by releasing cytokines such as interleukin 10 (IL-10) and transforming growth factor $\beta$ (TGF- $\beta$ ) [120]. In the tumor microenvironment, tumor cells also express regulatory molecules to increase tumor-suppressive immune cell populations, such as $T$ regulatory cells ( $T_{\text {regs }}$ ), and enable tumors to escape from the immune system [121]. Immunotherapeutic strategies are generally considered to display less unwanted toxicity compared with other types of cancer treatment. The development of anti-cancer immunotherapy is progressing in antigen-specific or antigen-nonspecific branches. Currently, the most promising immune therapy is the immune checkpoint inhibitors by blocking programmed cell death protein 1 (PD-1) and cytotoxic T lymphocyte antigen 4 (CTLA-4) [122]. However, neoplastic cells are capable of escaping 
from immune surveillance through the inhibitory feedback loops, including the PD-1 pathway. Particularly, the efficacy of checkpoint inhibitors to suppress lung cancer, especially advanced or metastatic non-small cell lung cancer (NSCLC) is modest [123].

A prophylactic cancer vaccine is a promising choice to reduce pulmonary malignancy. The majority of cancer vaccines under development usually need robust adjuvants to enhance the immune response because most of the tumor antigens targeted in vaccines are identical to "self" antigens [124]. However, the efficacy of a lung cancer vaccine will be markedly increased if the vaccine targets multiple "non-self" antigens presented only by lung tumors. Emerging evidence has demonstrated that tumor cells and embryonic stem cells (ESCs) share common antigens that are considered as "non-self" antigens, because they are not expressed in normal adult tissues [18]. Based on the antigenic similarity between malignant cells and ESCs, researchers have developed an prophylactic lung cancer vaccine composed of irradiated, intact murine ESCs and murine fibroblasts expressing the immunostimulatory adjuvant granulocyte-macrophage colony stimulating factor (GM-CSF) [29]. In support of cancer prevention capability of ESCs, a recent study has demonstrated that irradiated, induced pluripotent stem cells (iPSCs) along with the adjuvant CpG function as a tumor vaccine to elicit an anti-tumor response against transplanted breast cancer, mesothelioma and melanoma [30].

Despite the promise of ESC-based vaccine to evoke anti-lung cancer 
immune responses, such a vaccine has two obvious challenges to overcome for human application, First, the administration of intact ESCs, albeit irradiated, raises the risk of embryoma/teratoma formation. Furthermore, it is unnecessarily complicated to use fibroblasts as a source for the adjuvant GMCSF. To overcome these hurdles, we have developed an alternative prophylactic vaccine comprised of exosomes from murine ESCs engineered to produce GM-CSF (ESC-exo/GM-CSF). This self-contained, relatively stable exosome-based vaccine significantly slowed or blocked the outgrowth of subcutaneously implanted lung tumors (Figure 2. 6).

Metastasis results from the tumor cells on primary sites migrating to distant organs. The process of metastasis includes cancer cell proliferation, angiogenesis, cell adhesion, migration, and invasion into the surrounding tissue [125]. The prognosis of cancer patients decreases dramatically once metastasis happens [126]. Despite ongoing progress in diagnosis and patient care, metastasis is still the principal cause of mortality from neoplastic diseases. Lung metastasis occurs when malignant cells originating in another organ of the body migrate to and establish in the lung. Chemotherapy is the most commonly used therapy for lung metastasis with modest efficacy [127]. Therefore, if malignant cells metastasizing to the lung could be recognized by the immune system and eliminated before their establishment and growth in the lung, it would have promising application in clinics.

In this study, we show that ES-exo/GM-CSF vaccination effectively 
prevents the outgrowth of metastasized lung tumors in mice. Notably, the antitumor efficacy of this vaccination strategy is associated with reduced tumorpromoting immune cells and increased tumor-suppressing immune cells in lung metastases. Overall, our research provides a strategy for developing a cell-free preventative vaccine against metastasized lung tumors. 


\section{MATERIALS AND METHODS}

\section{Mice}

Female C57BL/6 mice ( 8 weeks of age) were purchase from Jackson Laboratory (Bar Harbor, ME) and housed at the University of Louisville Research Resources Facilities (RRF) under standard conditions. Mice were handled in accordance with the American Association for the Accreditation of Laboratory Animal Care (AAALC) guidelines and the "Guide for the Care and Use of Laboratory Animals" (Institute of Laboratory Animal Resources, National Research Council, National Academy Press, 1996). The mouse study was approved by the Institutional Animal Care and Use Committee (IACUC) of the University of Louisville (protocol number: 18301).

\section{Vaccination and tumor challenge}

Exosomes from ES-D3 cells were acquired as described in "MATERIALS AND METHODS" section of Chapter 2. Female C57BL/6 mice (8 weeks of age) were immunized twice (days 0 and 7 ) with vehicle only ( 1 x PBS), $225 \mu \mathrm{g}$ exosomes isolated from ES-D3 cells expressing the empty vector (ES-Exo) or $225 \mu \mathrm{g}$ exosomes isolated from ES-D3 cells stably-expressing GM-CSF (ES-Exo/GMCSF). Exosomes were injected subcutaneously (s.c.) in the right flank of mice. Following vaccination, mice were challenged with LLC cells $\left(0.15 \times 10^{6}\right)$ by tail vein injection on day 14. Immuno-analysis of splenocytes and tumor-infiltrating lymphocytes were carried out 5 weeks following LLC cell challenge. Lung 
tumorigenesis was examined 6 or 7 weeks after LLC cell injection.

\section{Histological analysis of lung tissues}

Lung tissue sections were prepared as previously described with some modifications [128]. Briefly, lung tissues were fixed in 10\% neutral phosphate buffered formalin for 24 hours at room temperature. After paraffin processing (TEK VIP; Sakura Finetek; Torrance, CA) and embedding (EG1160; Leica Biosystems; Buffalo Grove, IL), paraffin microtomy (RM2135; Leica Biosystems) was performed at 5 microns thickness of slide for each section. 3 sections were processed with 50 microns between each. For each biopsy core, three consecutive sections are placed on 3 slides (total of 9 sections per lung tissue) to assure detection of tumorigenesis in a whole lung. Slides of sections were deparaffinized and rehydrated in xylene, ethanol and deionized water. Slides were stained in hematoxylin (95057-844; VWR; Radnor, PA). After rinsing with deionized water, the slides were stained by eosin (HT110232; Thermo Fisher) and dehydrated in ethanol and xylene. Coverslips were mounted on slides by xylene-based permount ${ }^{\mathrm{TM}}$ mounting medium (SP15-500; Thermo Fisher; Waltham, MA). Finally, the slides were dried overnight in a chemical hood. The slides were scanned by Aperio Imagescope (Leica Biosystems) and analyzed with the software (version 12.3.3).

\section{Antibodies for immune-analysis}

Antibodies used for evaluating splenocytes and tumor-infiltrating lymphocytes 
were: anti- $\mathrm{CD} 3 \mathrm{mAb}$ (clone 17A2, 100222); anti-CD4 mAb (clone GK1.5; 100406); anti-CD8 mAb (clone 53-6.7; 100712); anti-CD11b mAb (clone M1/70; 101228); anti-Ly6C mAb (clone HK1.4; 128006); anti-Ly6G mAb (clone 1A8; 127608); anti-CD16/CD32 mAb (clone 2.4G2; 101320) from Biolegend (San Diego; CA), anti-CD44 mAb (clone IM7; 17-0441-82); anti-CD25 mAb (clone PC61.5; 45-0251-82) from (Thermo Fisher), anti-CD62L mAb (clone MEL-14; 553152; BD Biosciences; San Jose, CA).

\section{Immuno-analysis of splenocytes}

Five weeks following tail vein injection of LLC cells into female C57BL/6 mice, spleens were resected and splenocytes were acquired. Single splenocyte suspensions were stained with the indicated antibodies for 30 minutes after blocking with CD16/CD32 antibody for 15 minutes at $4{ }^{\circ} \mathrm{C}$. After washing, cell surface and intracellularly stained cells were analyzed on a FACSCalibur (Becton Dickinson; Franklin Lakes, $\mathrm{NJ}$ ) and results were analyzed using FlowJo software (TreeStar, Inc., Ashland, OR).

\section{Immuno-analysis of tumor-infiltrating immune cells}

Vaccinated and control mice with LLC tumors metastasized to lungs were euthanized 35 days after tumor challenge. Lungs were resected and metastasized LLC tumors were dissected and chopped into small pieces before incubation with a mixture of enzymes dissolved in HBSS, including collagenase type IV (400 U/ml; C9891; Sigma-Aldrich; St. Louis, MO), hyaluronidase (0.025 
mg/ml; H6254; Sigma-Aldrich) and DNase I (0.01 mg/ml; D5025; Sigma-Aldrich) for 2 hours at $37^{\circ} \mathrm{C}$ with occasional shaking. The resultant cells were washed and passed through a Ficoll gradient (17144002; GE Healthcare; Chicago, IL) to eliminate dead cells. Tumor-infiltrating lymphocytes (TILs) were then analyzed by flow cytometry for the expression of markers for different immune cells. Anti-CD45 antibody was used to selectively exclude $\mathrm{CD} 45^{-}$tumor cells from analysis so that only $\mathrm{CD} 45^{+}$immune cells were evaluated. The same number of cells (based on side-scatter and forward-scatter analyses) was acquired in all samples. Respective antibodies specific for the makers were used to quantitate the abundance of different immune cell types. T regulatory cells ( Tregs; $\left._{\text {Foxp }}{ }^{+}\right)$were analyzed using the anti-mouse Foxp3 staining kit (005523-00; Thermo Fisher).

\section{Intracellular cytokine staining}

To evaluate TNF- $\alpha$ and IFN- $\gamma$ production, TILs were harvested and restimulated for $6 \mathrm{~h}$ with the LLC lysate $(50 \mu \mathrm{g} / \mathrm{ml})$ in the presence of Golgiplug (555029; BD Biosciences) at a concentration of $1 \mu \mathrm{l} / \mathrm{ml}$ of culture medium. After restimulation, cells were harvested, Fc receptors were blocked using antiCD16/CD32 antibodies, and cells were stained for surface expression of CD8 and intracellular expression of cytokines using Cytofix/Cytoperm kit (555029; BD biosciences) according to the manufacturer's instructions and analyzed by flow cytometry. 


\section{Statistical analysis}

The statistical analysis was carried out using StatView version 5.0.1 software (Windows version; SAS Institute, Cary, NC) or GraphPad Prism 5.0 software (GraphPad Prisim Software, Inc., La Jolla, CA). Comparisons between experimental groups were conducted using Student's $\mathrm{t}$ test or one-way analysis of variance (ANOVA) where $P$ value $<0.05$ was considered significant. 


\section{RESULTS}

\section{Vaccination with ES-exo/GM-CSF inhibits metastasized lung tumor growth}

Metastatic lung cancer is one of the leading causes of death worldwide. As a low immunogenic cancer, it is resistant to the surveillance of the immune system [129, 130]. In our earlier studies, a prophylactic vaccine comprised of exosomes derived from murine ESCs engineered to produce GM-CSF (ESexo/GM-CSF) significantly slowed or blocked the outgrowth of subcutaneously implanted LLC (Figure 2.6). To evaluate the efficacy of ES-exo/GM-CSF vaccine in preventing metastasized lung tumor development, a mouse model of experimental pulmonary metastasis was investigated., In this model, tumor cells are first injected into the tail vein then reach the lung through blood circulation, where tumors are established [131].

Following a standard immunization timing regimen, C57BL/6 mice were vaccinated at day 0 and again on day 7 with 1 x PBS (vehicle control), exosomes alone (ES-exo) or GM-CSF-containing exosomes (ES-exo/GM-CSF) through subcutaneous (s.c.). injection (Figure 3.1A). Mice were then challenged with tail vein inoculation of LLC cells $\left(0.15 \times 10^{6}\right)$ at day 14 . In this experimental model, LLC tumors were established in the lung 5 weeks following LLC challenge.

To rigorously examine the vaccination efficacy of ES-exo/GM-CSF on 
metastasized LLC tumors, we carried out two independent experiments to examine the status of lung tumors generated by metastasized LLC cells 6 weeks or 7 weeks following initial LLC cell administration. Immediately after collecting lung tissues from the mice in 3 experimental groups, we first counted the numbers of tumor nodules on lung surface [132], as a lung tumor nodule is defined as a discrete, well-defined, rounded opacity that is completely surrounded by lung tissue (Figure 3.1B). The results of lungs acquired 6 weeks after LLC inoculation are similar to those obtained 7 weeks post LLC injection (Figure 3.1C). Examination of resected lungs revealed that the numbers of lung tumor nodules in mice vaccinated with ES-exo/GM-CSF were significantly smaller than those in mice injected with vehicle control. The majority of PBSadministered mouse lungs had surface tumors (8 out of 9 for 6-week group; 10 out of 11 for 7 -week group) with an average of about 3.5 lesions for each mouse. In contrast, mice vaccinated with ES-exo/GM-CSF only had an average of 0.5 lung tumors/mouse (6-week group) or 1.4 lung tumors/mouse (7-week group), indicating that immunization with ES-exo/GM-CSF inhibited the metastasized lung tumor development. The average tumor nodule number in mice vaccinated with ES-exo was higher compared with ES-exo/GM-CSF-immunized mice, but the difference was not significant due to variations among the mice in each group.

To further investigate the efficacy of this vaccination strategy, total lung tumor mass of metastasized LLC was evaluated by a histological approach. 
Serial sections were produced from each lung resected from the mice, stained with hematoxylin and eosin (H\&E) and analyzed by measuring the tumor lesion areas in each lung tissue (Figure 3.1D). Typical lung sections from mice showed a striking difference between PBS-inoculated mice versus those receiving ESexo/GM-CSF vaccination. Numerous large tumor lesions were detected in nonvaccinated control mouse lung sections, whereas vaccinated animals were almost absent of any detectable lesions (Figure 3.1D). The tumor burden was calculated as the percentage of lung area occupied by tumor lesions in each slide. As shown in Figure 3.1 E, ES-exo/GM-CSF-vaccinated mice had a significantly smaller percentage of tumor-bearing lung area compared with the mice in unvaccinated control (1x PBS) group or the mice vaccinated with exosomes without GM-CSF (ES-exo). Absence of metastasized lung tumors in mice immunized with ES-exo/GM-CSF provides evidence that ES-exo/GMCSF suppresses the development of metastasized lung malignancy, which is in agreement with our earlier studies with subcutaneously implanted LLC tumors (Figure 2.6). 
A

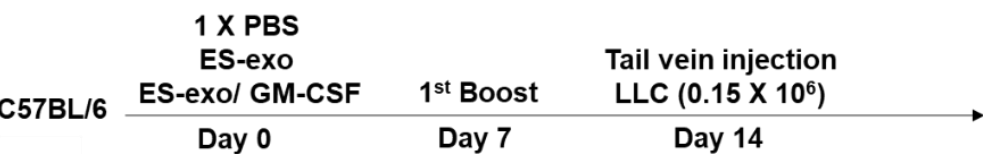

B

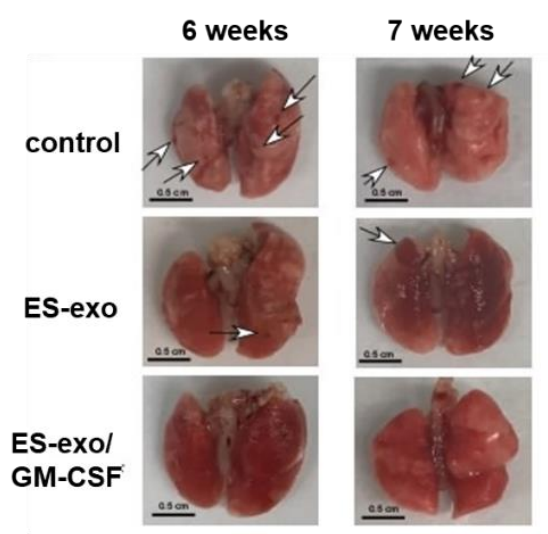

D

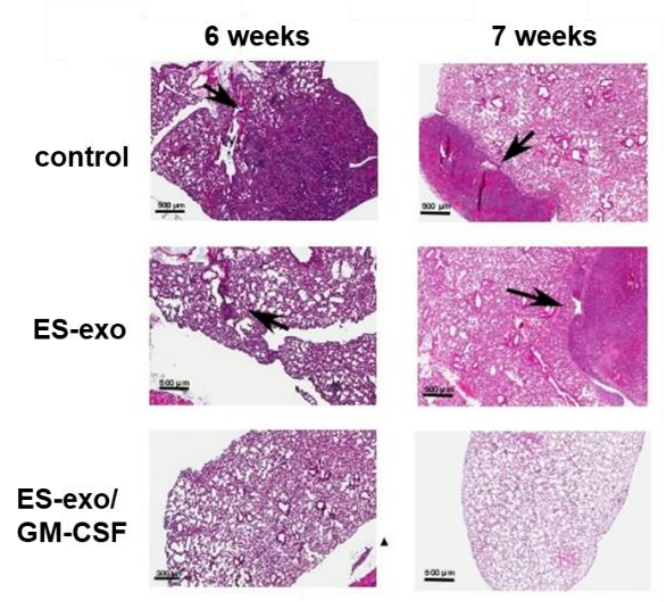

C
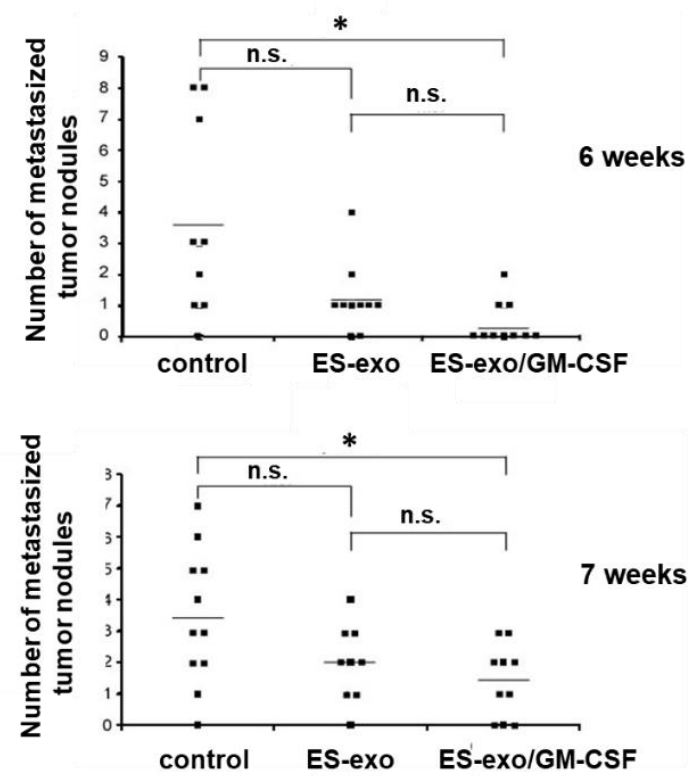

E
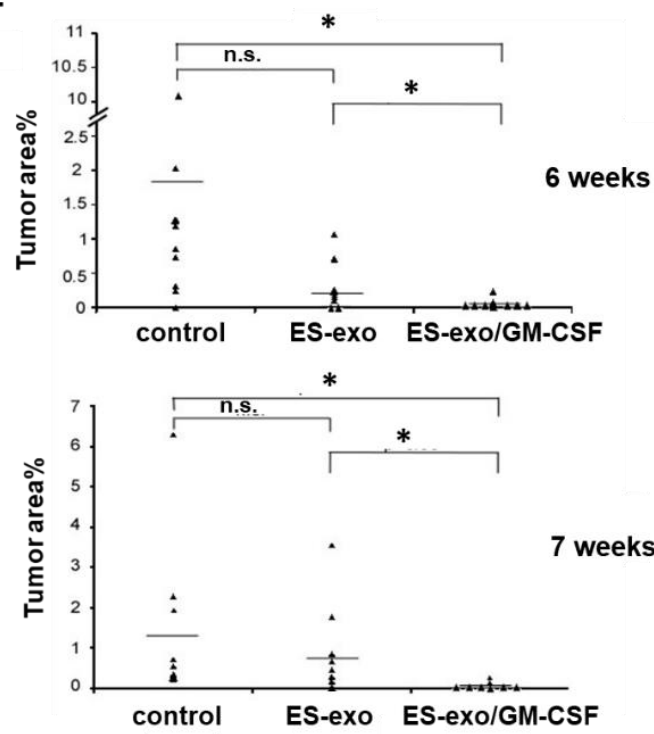

Figure 3.1. ES-exo/GM-CSF vaccination inhibits the outgrowth of metastasized lung tumors.

(A) The scheme of vaccination is depicted. Female C57BL/6 mice were immunized twice (Days 0 and 7 ) with vehicle control (1 x PBS), exosomes 
isolated from vector control ES-D3 cells (ES-exo) or exosomes isolated from GM-CSF-expressing ES-D3 cells (ES-exo/GM-CSF) prior to tail vein injection with LLC cells $\left(0.15 \times 10^{6}\right)$ on Day 14 . Lung tumor development was examined 6 weeks or 7 weeks after LLC injection. (B) Representative images of lungs resected from euthanized mice. Surface tumor nodules were indicated by arrows. Scale bar, $0.5 \mathrm{~cm}$. (C) Surface tumor nodules of resected lungs were enumerated by inspection. The data are presented as a dot graph of the number of surface tumor nodules of lungs. In the experiments carried out 6 weeks after LLC injection, 10 mice in each group. In the studies performed 7 weeks following LLC challenge, 11 mice in control group, 9 mice in ES-exo group and 10 mice in ES-exo/GM-CSF group. ${ }^{*}, p$ $<0.05 ;{ }^{* *}, \mathrm{p}<0.01$; ANOVA with Tukey's multiple comparison test. (D) The histological sections of resected lungs were examined by H\&E staining. Representative images of lung sections are shown (magnification: $x$ 200). Lesions on lung sections are indicted by arrows. Scale bar, $500 \mu \mathrm{M}$. (E) The tumor lesion areas of each lung tissue sections were measured. The percentage of total lung area taken up by lung tissues was quantified from measurements on H\&E sections of resected lungs from animals in each group. For each lung, the average value of 3 sections with 50 microns apart was calculated. ${ }^{*}, \mathrm{p}<0.05 ;{ }^{* *}, \mathrm{p}<0.01$; ANOVA with Tukey's multiple comparison test. 


\section{ES-exo/GM-CSF vaccination decreases $T$ regulatory cells (Tregs) in lung metastases.}

It is generally believed that immune cells infiltrating into tumor tissues play essential roles in tumor development and progression [133]. Importantly, tumorinfiltrating immune cells are involved in cancer prevention efficacy of a vaccine composed of intact ESCs and STO fibroblasts expressing GM-CSF [29]. As shown in Figure 3.1, a limited number of mice vaccinated with ES-exo/GM-CSF did develop LLC lesions in the lung. To elucidate the underlying mechanisms of the immunity against metastasized lung cancer by ES-exo/GM-CSF vaccination, Dr. Kavitha Yaddanapudi's laboratory analyzed the phenotypes of tumor-infiltrating immune cells by examining the expression of different immune cell markers. Pan-leukocyte marker CD45 was studied to ensure that only CD45 ${ }^{+}$immune cells in tumor infiltrates were examined.

T cells are known to play an important role in immune responses arising during malignancy. Among them, cytotoxic $T$ cells $\left(T_{c}\right)$ have the capability to kill a variety of neoplastic cells, whereas immunosuppressive $T$ regulatory cells ( $T_{\text {regs }}$ ) contribute to the development and progression of many cancer types by reducing effector cell responses $[134,135]$. Therefore, we first analyzed the effects of our vaccination strategy on tumor-infiltrating $T_{c}$ and $T_{\text {regs. }}$ As shown in Figures $3.2 \mathrm{~A}$ and $3.2 \mathrm{~B}$, immunization with ES-exo/GM-CSF failed to affect intratumor $\mathrm{CD}^{+} \mathrm{T}_{c}$ levels. However, flow cytometry analysis revealed a significant decrease in the percentage of Foxp3 ${ }^{+} \mathrm{T}_{\text {regs }}$ in $\mathrm{CD}^{+}{ }^{+} \mathrm{T}$ helper cells of 
tumor infiltrates from ES-exo/GM-CSF-vaccinated mice when compared with non-vaccinated control mice (Figures 3.2C-D).

A

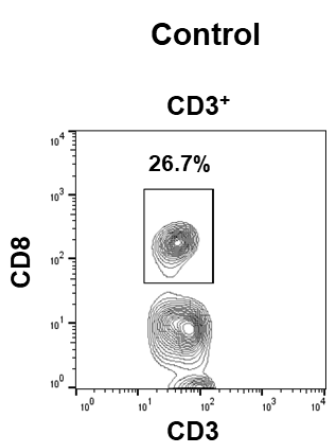

C

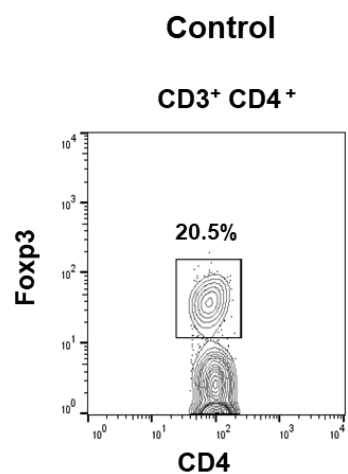

ES-exo/GM-CSF

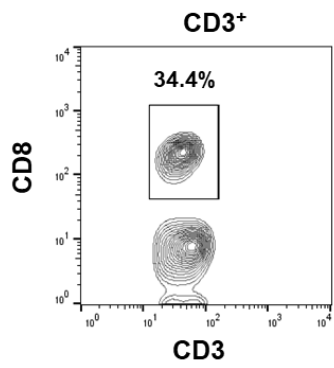

ES-exo/GM-CSF

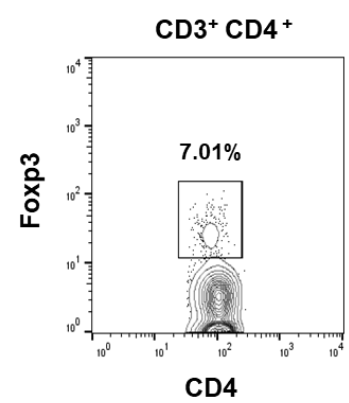

B

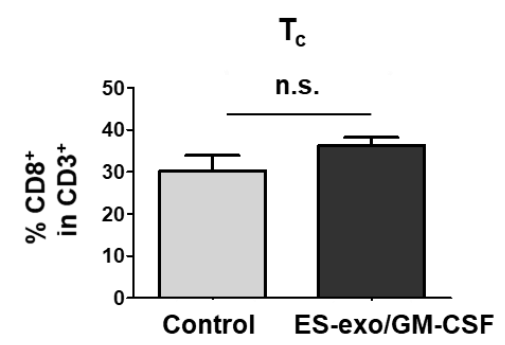

D

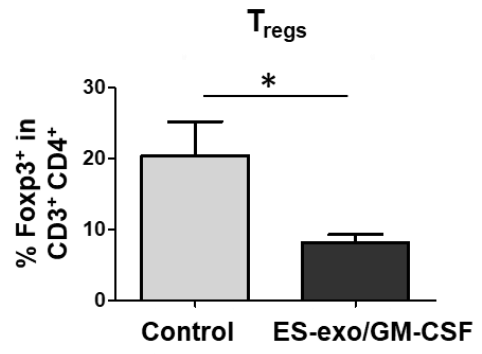


Figure 3.2. Vaccination with ES-exo/GM-CSF decreases $\mathrm{T}$ regulatory cells ( Tregs $_{\text {s }}$ in metastasized lung tumors.

Female C57BL/6 mice were vaccinated twice (days 0 and 7) with $1 \times$ PBS (control) or ES-exo/GM-CSF prior to tail vein injection with LLC on day 14. Five weeks following LLC challenge, lung tumors were resected, digested by enzymes, and tumor-infiltrating cells were harvested and analyzed by flow cytometry. The pan-hematopoietic marker CD45 was used to identify intratumoral immune cells. (A) The presence of tumor-infiltrating cytotoxic T cells $\left(T_{c}\right)$ was examined and the percentages of $C D 8^{+} T_{c}$ cells in $C D 3^{+} T$ cell population were determined. (B) Bar graphs showing average of percentages of tumor-infiltrating $\mathrm{CD} 8^{+} \mathrm{T}_{\mathrm{c}}$ cells in $\mathrm{CD} 3^{+} \mathrm{T}$ cells. Four mice in control group, and six mice in ES-exo/GM-CSF group. Mean \pm SD, n.s., not significant; ANOVA with Tukey's multiple comparison test. (C) Tumorinfiltrating Foxp $3^{+} \mathrm{T}_{\text {regs }}$ in $\mathrm{CD} 3^{+} \mathrm{CD} 4^{+} \mathrm{T}$ cells obtained from control and ESexo/GM-CSF-vaccinated mice were evaluated. Numbers in the plots represent the percentages of subpopulations. (D) Summary of the data shown in (C). ( $n=4$ in control group, $n=6$ in ES-exo/GM-CSF group; mean \pm SD, * $p<0.05$; ANOVA with Tukey's multiple comparison test). 


\section{Vaccination with ES-exo/GM-CSF suppresses tumor-infiltrating myeloid derived suppressor cells (MDSCs).}

In addition to $T_{\text {regs, }}$ myeloid-derived suppressor cells (MDSCs) are another prominent suppressor class hampering anti-tumoral effector responses [136].

To elucidate the immunomodulatory influences of ES-exo/GM-CSF vaccination, we first evaluated the abundance of $C D 11 b^{+}$immune cells in lung metastases with the help of Dr. Kavitha Yaddanapudi. The presence of CD11 $b^{+}$MDSC in tumor-infiltrating non- (CD3 $^{-}$) and non-B (CD19-) immune cells was significantly reduced from $80.7 \%$ to $34.9 \%$ (Figure $3.3 \mathrm{~A}$ ). As a heterogeneous population of immature myeloid cells, MDSCs are composed of the monocytic subset (M-MDSCs) and granulocytic subset (G-MDSCs), both of which display immune-suppressive capability to promote tumor development. To further explore the effects of ES-exo/GM-CSF vaccination on intratumoral MDSCs, we examined the presence of MDSC subsets in metastasized lung tumors. As shown in Figure 3.3C, the percentage of tumor-infiltrating Gr-1+ G-MDSCs in CD11 ${ }^{+}$non-T and non-B immune cells was significantly decreased from $49.5 \%$ to $15.5 \%$ in mice vaccinated with ES-exo/GM-CSF and challenged with LLC cells when compared with non-vaccinated, LLC challenged control mice. In contrast, ES-exo/GM-CSF vaccination did not reduce the percentage of Gr-1 ${ }^{\text {low }}$ M-MDSCs in lung metastases, suggesting that different subclasses of intratumoral MDSCs play distinct role in anti-tumor efficacy of ES-exo/GM-CSF vaccination. 
A

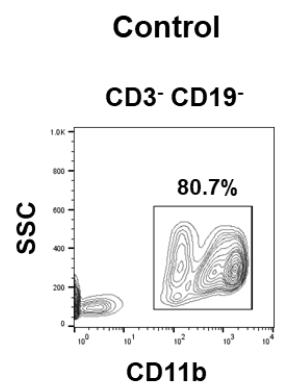

C

Control

CD3- CD19- CD11b+

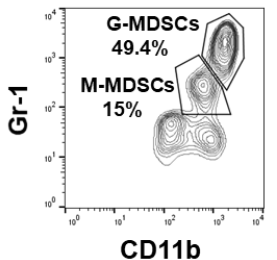

ES-exo/GM-CSF

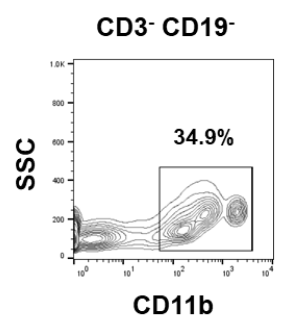

ES-exo/GM-CSF

CD3-CD19- CD11b+

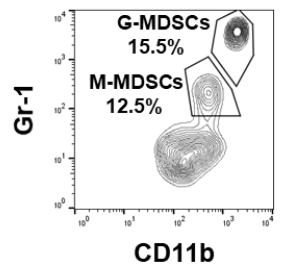

B

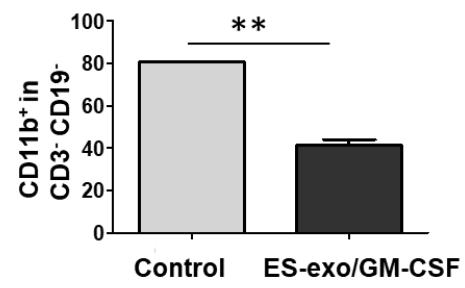

D

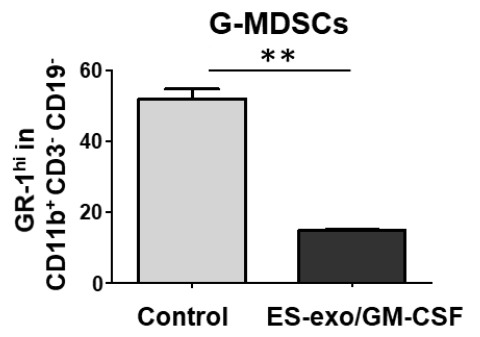

E

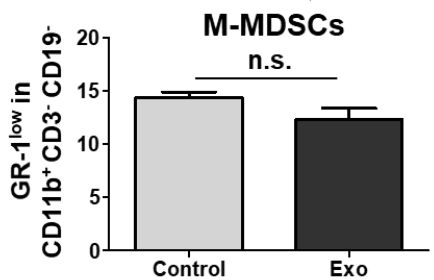


Figure 3.3. Vaccination with ES-exo/GM-CSF suppresses tumorinfiltrating MDSCs

Female C57BL/6 mice were vaccinated with 1 x PBS or ES-exo/GM-CSF twice (days 0 and 7 ) and challenged by tail vein inoculation LLC one week later. Myeloid derived suppressor cells (MDSCs) infiltrating into lung tumors were examined 5 weeks after LLC injection. Intratumoral immune cells were identified by CD45, a pan-hematopoietic marker. (A) Dot plots showing the abundance of $\mathrm{CD} 11 \mathrm{~b}^{+}$cells in tumor-infiltrating non- $\mathrm{T}\left(\mathrm{CD} 3^{-}\right)$and non-B (CD19-) immune cells obtained from control and ES-exo/GM-CSFvaccinated mice. The percentages of the individual subpopulation are shown. (B) The data shown in (A) are summarized with 4 mice in control group, 6 mice in ES-exo/GM-CSF group. Mean \pm SD, ${ }^{* *}, p<0.001$; ANOVA with Tukey's multiple comparison test. (C) Gr-1 ${ }^{\text {high }}$ granulocytic myeloid derived suppressor cells (G-MDSCs) and Gr-1 ${ }^{\text {low }}$ Monocytic myeloid derived suppressor cells (M-MDSCs) in $\mathrm{CD}_{11} \mathrm{~b}^{+}$populations shown in $(\mathrm{A})$ were examined. The percentages of each subpopulation are indicated in the graphs. (D-E) Bar graphs show the summarized data presented in (C). $(n=$ 4 in control group, $\mathrm{n}=6$ in ES-exo/GM-CSF group; mean $\pm \mathrm{SD} ;{ }^{* * *}, \mathrm{p}<$ 0.0001 ; n.s., not significant; ANOVA with Tukey's multiple comparison test). 


\section{Vaccination with ES-exo/GM-CSF reduces the percentage of macrophages in metastasized lung tumors.}

As an integral component of tumor microenvironment, intratumoral macrophages are associated with initiation as well as progression of various malignancies [137]. The influence of vaccination with ES-exo/GM-CSF on tumor-infiltrating macrophages was investigated by Dr. Kavitha Yaddanapudi. Previous studies demonstrate that the cell surface protein $\mathrm{F} 4-80$ is a major marker of murine macrophages [138]. As shown in Figure 3.4A, the abundance of $\mathrm{F} 4-80^{+} \mathrm{Gr}-1^{-}$macrophages in intratumoral $\mathrm{CD} 11 \mathrm{~b}^{+}$non-T and non-B immune cells was significantly decreased from $15.8 \%$ to $3.06 \%$ in ES-exo/GM-CSFvaccinated mice compared with non-vaccinated mice inoculated with the vehicle control. The $\mathrm{CX} 3 \mathrm{CR} 1^{+}$subpopulation of macrophages has been shown to play a central role in lung cancer growth and metastasis via CX3CR1 signaling [139]. However, the percentage of tumor-infiltrating CX3CR1 ${ }^{+}$ macrophages was not significantly influenced by vaccination with ES-exo/GMCSF (Figure 3.4D), suggesting that the involvement of distinct subpopulations of intratumoral macrophages in the immune responses to ES-exo/GM-CSF vaccination is different. 
A

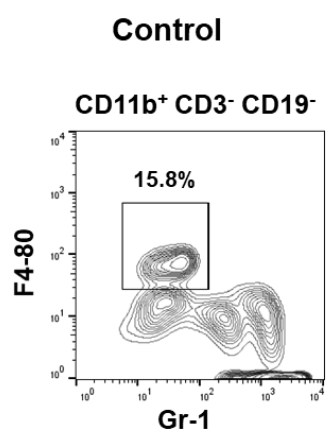

C

Control

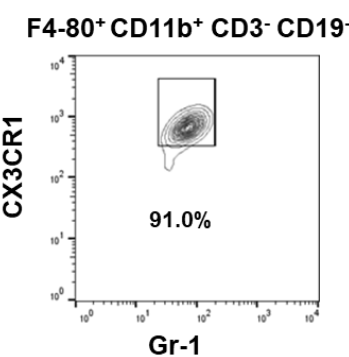

ES-exo/GM-CSF

CD11b+ CD3- CD19-

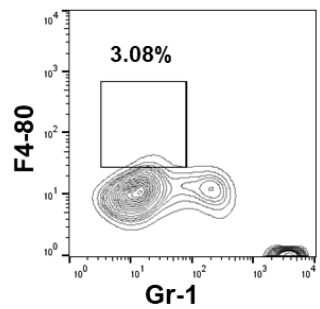

D

ES-exo/GM-CSF

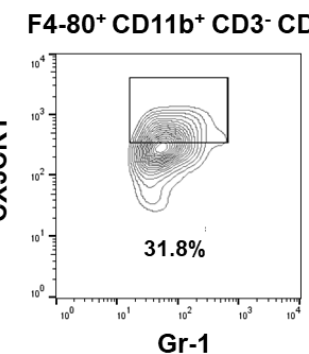

B $\mathrm{F}_{480^{+}}$macrophages
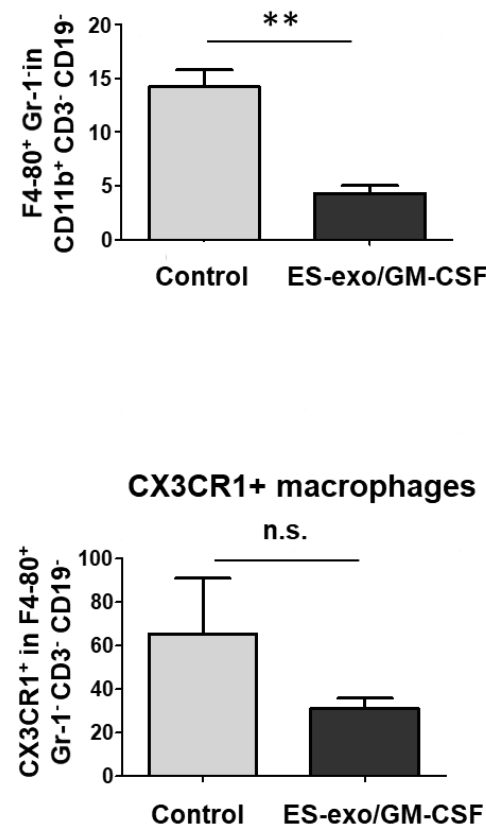
Figure 3.4. Vaccination with ES-exo/GM-CSF reduces tumor-infiltrating macrophages.

The vehicle control (1 x PBS) or ES-exo/GM-CSF was injected into female C57BL/6 mice twice with 7 days apart, followed by tail vein inoculation of LLC 7 days later. The presence of tumor-infiltrating macrophages was analyzed by flow cytometry 5 weeks after LLC injection. CD45, as a panhematopoietic marker, was analyzed to evaluate intratumoral immune cells. (A) The percentages of $\mathrm{F} 4-80^{+} \mathrm{GR}-1^{-}$macrophages in intratumoral non- $\mathrm{T}$ (CD3-) and non-B (CD19-) immune cells from control and ES-exo/GM-CSFvaccinated mice are shown. Numbers in the plots represent the percentages of each subpopulation. (B) Bar graphs showing the average of percentages of F4-80+GR-1- macrophages obtained from control and ES-exo/GM-CSFvaccinated mice ( $\mathrm{n}=4$ in control group, $\mathrm{n}=6$ in ES-exo/GM-CSF group; mean $\pm \mathrm{SD},{ }^{* *}, \mathrm{p}<0.001$; ANOVA with Tukey's multiple comparison test). (C) Dot plots representing the abundance of $\mathrm{CX} 3 \mathrm{CR} 1^{+}$macrophage subset in tumor-infiltrating general macrophage populations shown in (A). The percentages of the individual subpopulation are indicated. (D) The data shown in (C) are summarized with 4 mice in control group, 6 mice in ESexo/GM-CSF group. Mean \pm SD, n.s., not significant; ANOVA with Tukey's multiple comparison test. 
5. ES-exo/GM-CSF vaccination promotes intratumoral B cell populations.

Emerging evidence has demonstrated that tumor-infiltrating B cells play a critical role in tumor development at all stages of lung tumorigenesis [140]. To test the effect of ES-exo/GM-CSF vaccination on the levels of B cell populations in metastasized lung tumors, Dr. Kavitha Yaddanapudi's group evaluated the presence of $\mathrm{CD} 19^{+} \mathrm{B}$ cells in intratumoral cells positive for CD45. As shown in Figure 3.5, the percentage of $\mathrm{CD} 19^{+} \mathrm{B}$ cells in tumor-infiltrating CD45+ immune cells was significantly increased from $11.1 \%$ to $38.1 \%$ in mice vaccinated with ES-exo/GM-CSF and challenged with LLC cells than their non-vaccinated control counterparts. 
A

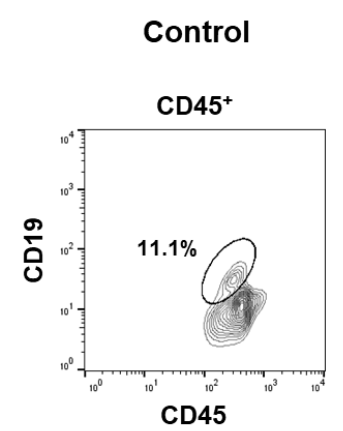

ES-exo/GM-CSF

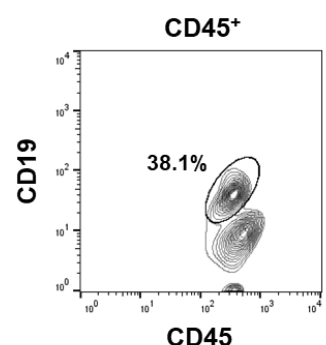

B

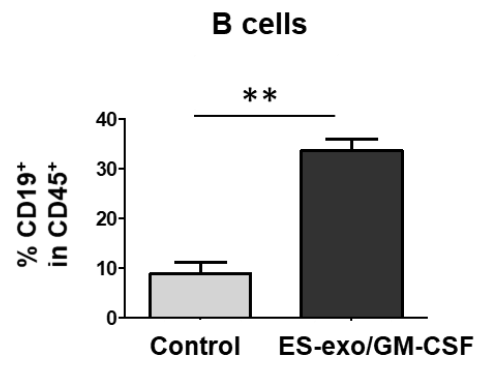

Figure 3.5. ES-exo/GM-CSF vaccination promotes tumor-infiltrating $B$ cells against lung metastases.

Female C57BL/6 mice were vaccinated twice (days 0 and 7) with vehicle only (1 1 PBS) or ES-exo/GM-CSF followed by LLC challenge through tail vein 7 days later. After 5 weeks, cells infiltrating into lung metastases were harvested and evaluated using the pan-hematopoietic marker CD45 to distinguish tumor-infiltrating immune cells from malignant cells by a flow cytometry analysis. (A) Dot plots showing the percentages of CD19+ B cells in $\mathrm{CD}_{4} 5^{+}$intratumoral cells obtained from control and ES-exo/GM-CSFvaccinated mice. Numbers in the dot plots are the percentages of each subpopulation. (B) Bar graphs showing average of percentages of CD19+B cells in tumor-infiltrating $\mathrm{CD}_{4} 5^{+}$cells acquired from control and ES-exo/GMCSF-vaccinated mice with 4 mice in control group, 6 mice in ES-exo/GMCSF group. Mean \pm SD, ${ }^{* *}, p<0.001$; ANOVA with Tukey's multiple comparison test. 


\section{Intratumoral Th1 and Th17 effector cells are not affected by ES-exo/GM- CSF vaccination.}

It is well known that tumor-infiltrating $\mathrm{CD}^{+}$effector $\mathrm{T}$ cells play critical roles in malignancies. Among them, Th1 and Th17 subsets of $\mathrm{CD} 4^{+}$cells are deeply associated with pulmonary tumorigenesis [141, 142]. To investigate whether ES-exo/GM-CSF vaccination affects the presence of Th1 or Th17 populations in metastasized lung tumors, Dr. Kavitha Yaddanapudi's laboratory helped us to examine $\mathrm{CX} 3 \mathrm{CR} 1^{+}$Th1 and $\mathrm{ROR} \gamma \mathrm{T}^{+}$Th17 cells in tumor-infiltrating $\mathrm{T}$ cells positive for CD4. As shown in Figure 3.6, the percentages of intratumoral CX3CR1 ${ }^{+}$Th1 and ROR $\gamma \mathrm{T}^{+}$Th17 cells $\mathrm{CD}^{+}$effector $\mathrm{T}$ cells were not significantly altered in mice vaccinated with ES-exo/GM-CSF and challenged with LLC cells compared with non-vaccinated control mice.

A

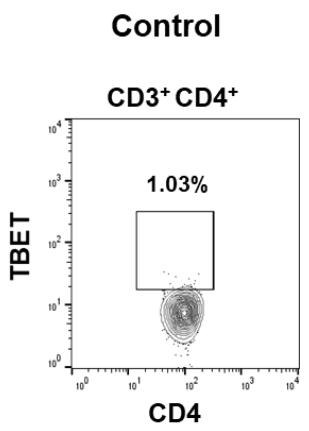

C

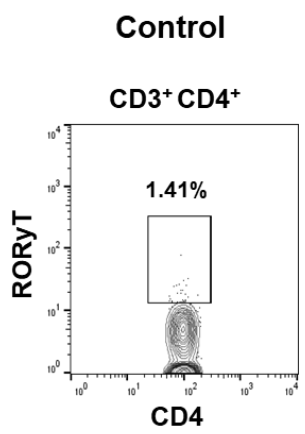

ES-exo/GM-CSF

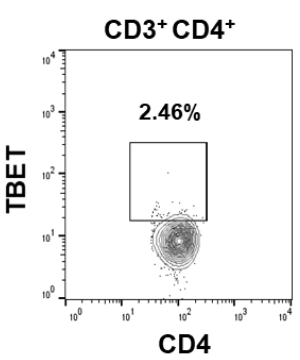

ES-exo/GM-CSF

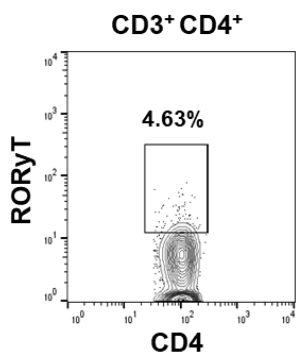

B

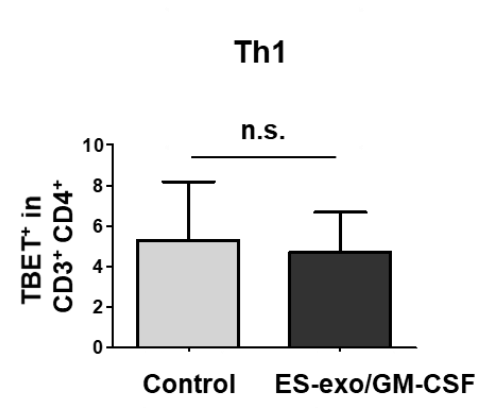

D

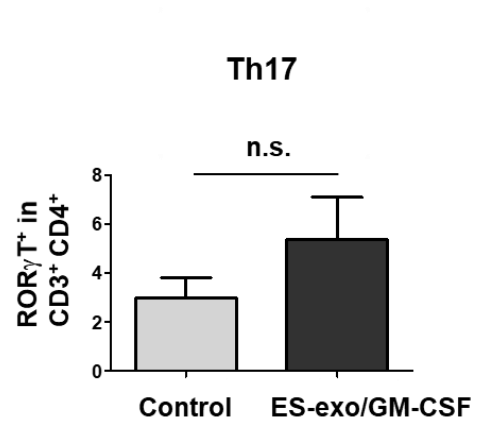


Figure 3.6. The levels of Th1 and Th17 effector cells in lung metastases are not affected by ES-exo/GM-CSF vaccination.

Female C57BL/6 mice were vaccinated with 1 x PBS or ES-exo/GM-CSF twice (days 0 and 7). Seven days later, mice were injected with LLC cells through tail vein. Intratumoral $\mathrm{CD}^{+}{ }^{+}$Th1 and $\mathrm{CD}^{+}{ }^{+}$Th17 cells were examined 5 weeks following LLC inoculation. The pan-hematopoietic marker CD45 was evaluated to distinguish immune cells from cancer cells in lung metastases. (A) The presence of $\mathrm{TBET}^{+} \mathrm{Th} 1$ in tumor-infiltrating $\mathrm{CD}^{+} \mathrm{CD}^{+}$effector $\mathrm{T}$ cells was examined by flow cytometry. Numbers shown in plots are the percentage of each subpopulation. (B) Bar graphs showing average of percentages of $\mathrm{TBET}^{+}$Th1 cells in tumor-infiltrating $\mathrm{CD}^{+} \mathrm{CD}^{+}$cells acquired from control and ES-exo/GM-CSF-vaccinated mice with 4 mice in control group, 6 in ES-exo/GM-CSF group. Mean \pm SD, n.s., not significant; ANOVA with Tukey's multiple comparison test. (C) Intratumoral ROR $\gamma \mathrm{T}^{+}$Th17 cells in $\mathrm{CD}^{+} \mathrm{CD} 4^{+}$effector $\mathrm{T}$ cells obtained from control and ES-exo/GM-CSF-vaccinated mice were evaluated. Numbers in the plots are the percentages of subpopulations. (D) The data shown in (C) are summarized. ( $n=4$ in control group, $n=6$ in ES-exo/GM-CSF group; mean \pm SD, n.s., not significant; ANOVA with Tukey’s multiple comparison test). 


\section{Vaccination with ES-exo/GM-CSF induces a tumor cell-specific cytokine response in tumor-infiltrating $\mathrm{CD}^{+} \mathrm{T}$ cells.}

Since cytolytic cytokines produced by tumor-infiltrating $\mathrm{CD} 8^{+} \mathrm{T}$ cells are known to contribute to the anti-tumor function of $\mathrm{CD} 8^{+} \mathrm{T}$ cells [143], With the help of Dr. Kavitha Yaddanapudi's group, we investigated the capability of intratumoral $\mathrm{CD}^{+} \mathrm{T}$ cells from vaccinated mice to generate cytokines required for cytotoxic activity against tumors. Tumor-infiltrating immune cells were acquired from metastasized lung tumors in mice immunized with ES-exo/GM-CSF or the vehicle control. Following stimulation with LLC cell lysate, IFN- $\gamma$ and TNF- $\alpha$ levels in intratumoral $\mathrm{CD}^{+} \mathrm{T}$ cells were evaluated. As shown in Figure 3.7, a significantly higher percentage of IFN- $\gamma$-producing $\mathrm{CD} 8^{+} \mathrm{T}$ cells were found in lung metastases in ES-exo/GM-CSF-vaccinated mice than their nonvaccinated counterparts. However, the modest increase in IFN- $\gamma$-producing $\mathrm{CD}^{+} \mathrm{T}$ cells promoted by ES-exo/GM-CSF vaccination was not significant.

A

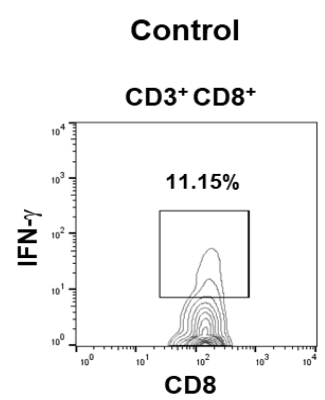

C

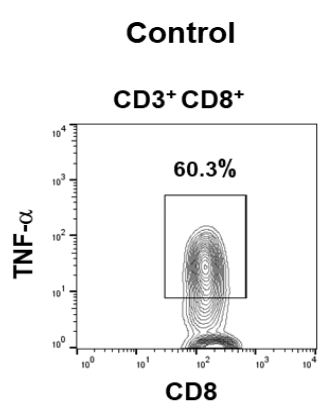

ES-exo/GM-CSF

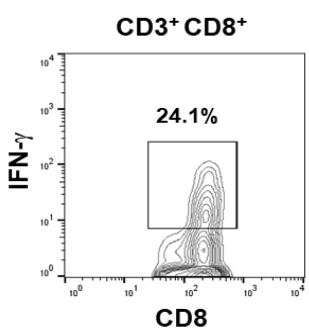

ES-exo/GM-CSF

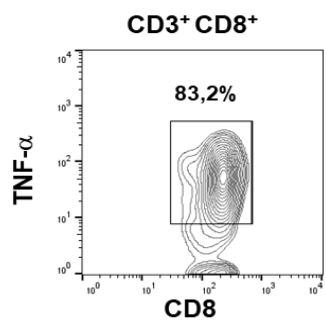

B

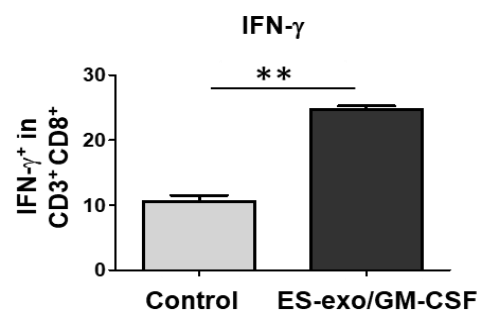

D

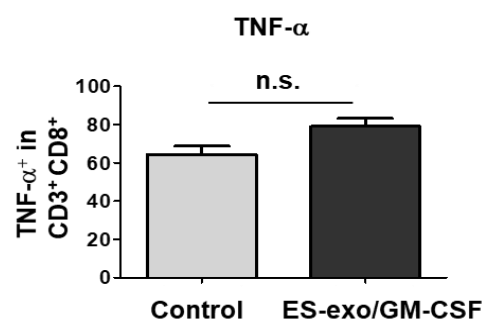


Figure 3.7. Vaccination with ES-exo/GM-CSF promotes cytokine production from $\mathrm{CD8}^{+} \mathrm{T}$ cells.

Female C57BL/6 mice were immunized twice (days 0 and 7) with vehicle control (1 $\mathrm{x}$ PBS) or ES-exo/GM-CSF prior to tail vein injection with LLC. 5 weeks after LLC challenge, lung tumors were resected, tumor-infiltrating cells were harvested and stimulated with LLC cell lysate for 6 hours. Intratumoral immune cells were identified by the pan-hematopoietic marker CD45 and intracellular expression of IFN-y and TNF- $\alpha$ was examined by flow cytometry. (A) Dot plots showing IFN-y expression in tumor-infiltrating $\mathrm{CD}^{+} \mathrm{CD}^{+} \mathrm{T}$ cells obtained from control and ES-exo/GM-CSF-vaccinated mice. Numbers in plots represent the percentages of each subpopulation. (B) The data shown in (A) are summarized with 4 mice in control group, 6 mice in ES-exo/GM-CSF group. Mean $\pm \mathrm{SD},{ }^{* *}, \mathrm{p}<0.001$; ANOVA with Tukey's multiple comparison test. (C) The presence of TNF- $\alpha^{+}$cells in tumorinfiltrating $\mathrm{CD} 45^{+} \mathrm{CD} 3^{+} \mathrm{CD} 8^{+}$cells was examined by flow cytometry. Numbers indicated in plots are the percentage of each subpopulation. (D) Bar graphs showing average of percentages of $\mathrm{TNF}-\mathrm{a}^{+}$in intratumoral $\mathrm{CD}^{+}{ }^{+} \mathrm{CD} 8{ }^{+} \mathrm{T}$ cells derived from control and ES-exo/GM-CSF-vaccinated mice. $\left(n=4\right.$ per group; mean $\pm S D$; ${ }^{* *}$, n.s., not significant; ANOVA with Tukey's multiple comparison test). 


\section{DISSCUSION}

The prevalence of pulmonary metastases from different primary tumors is relatively high due to the abundant bloodstream in the lung capillary bed [144]. Although there are several therapeutic options available for lung metastases, the overall outcome is still unsatisfactory. In this study, we investigated an experimental metastasis model where syngeneic LLC cells were administered directly into the bloodstream of wild-type C57BL/6 mice through tail vein injection, primarily resulting in pulmonary metastases [145]. Specifically, we examined the effects of a vaccine composed of exosomes from GM-CSFexpressing ES-D3 cells on metastasized lung malignancy. Our data provide evidence that a vaccine derived from GM-CSF-expressing human ESCs could potentially be used to prevent the development of lung metastases in the future.

In humans, metastasized lung malignancies are developed when cancer spreads to lung from where it originates. The metastasis mouse model in our study recapitulates the critical process of tumor cell extravasation from blood vessels in target organs, but it lacks the process of tumor cell metastasizing from primary tumor site and the generation of adhesion and traction forces required for cell migration [146]. Future research is warranted to investigate the efficacy of ES-exo/GM-CSF in tumor models more truthfully recapitulating the process of metastasis in lung cancer patients.

Accumulating evidence indicate that stem-like cells, called cancer stem cells (CSCs) and more differentiated trophoblast-like cells coexist within lung 
metastases. With self-renewal and differentiation capabilities similar to those of normal stem cells, CSCs are a likely source of cancer metastases. [147]. Furthermore, the presence of CSCs is a particular challenge in treating metastasized lung cancer because CSCs tend to be resistant to standard chemotherapy and radiotherapy. If lung CSCs could be recognized and eliminated by the immune system, the morbidity of cancer metastasis would likely decrease. Earlier studies suggest that the efficacy of ESC vaccination is associated with the immunity targeting CSCs [29]. In our study, vaccination with exosomes derived from ESCs induced immune responses against initiation and progression of lung metastases (Figure 3.1). It is conceivable that ES-exo/GMCSF vaccination triggers immune recognition and eradication of lung CSCs. Future studies are needed to address whether lung CSCs are the targets for this vaccination strategy against metastasized lung tumors.

Emerging evidence has demonstrated that tumor-infiltrating lymphocytes (TILs) are deeply involved in metastasis of malignancy [133]. As ES-exo/GMCSF vaccination successfully blocked the outgrowth of metastasized lung tumors (Figure 3.1), we evaluated the comprehensive profile of intratumoral immune cells to elucidate the nature of immunity against lung metastases evoked by ES-exo/GM-CSF vaccination. In our research, we first observed a significant reduction in the presence of $T_{\text {regs }}$ in lung metastases of vaccinated/tumor-challenged mice compared with non-vaccinated/tumorchallenged control mice though Th17 cells which are relevant to suppress the 
$T_{\text {regs }}$ differentiation were unchanged after immunization (Figure 3.2; 3.6). This is consistent with the findings that increased $T_{\text {regs }}$ population in metastasized malignancy often correlates with an immunosuppressive phenotype and poor patient prognosis [148]. Similarly, ES-exo-GM-CSF vaccination significantly decreased the abundance of MDSCs, particularly G-MDSC subset, in TILS (Figure 3.3). As a suppressor of $\mathrm{T}$ cell proliferation and activation, MDSCs expansion in TILs is an obstacle in tumor immunotherapy. Furthermore, it has been reported that the pulmonary G-MDSC infiltrates are dramatically increased as the metastases form in the lungs to suppress the anti-tumor immune response[149]. Therefore, the presence of two prominent suppressor populations that hamper anti-tumoral effector responses, Tregs and MDSCs, were suppressed by ES-exo/GM-CSF vaccination, which is correlated with the effectiveness of this vaccine.

Tumor-associated macrophages are one of the major constituents that promote tumor progression by inhibiting tumor cell apoptosis and producing many pro-angiogenic factors [150]. Intratumoral macrophages have been linked to poor clinical outcome in lung cancer patients $[137,151]$. In our study, percentage of $\mathrm{F} 4 / 80^{+}$macrophages in TILs was significantly decreased by immunization of ES-exo/GM-CSF compared with the control group (Figure 3.4B). As one subtype of macrophages, $\mathrm{CX} 3 \mathrm{CR} 1^{+}$macrophages are involved in lung cancer growth and metastasis [139]. However, we failed to observe any significant difference in $\mathrm{CX} 3 \mathrm{CR} 1^{+}$populations between the mice in the 
vaccinated group and their counterparts in the control group (Figure 3.4D).

Like many other intratumoral immune cells, macrophages infiltrated into tumors also display multifaceted activities to regulate tumor immunity, rather than simply stimulating or hindering tumorigenesis and metastasis [152]. There are two distinct subtypes of macrophages existing in tumor infiltrates: proinflammatory, anti-tumor M1 class and anti-inflammatory, pro-tumor M2 class. In the majority of solid tumors, M2 macrophages are considered as the dominant subtype, conferring an immunosuppressive microenvironment to support malignancy progression. However, the composition of tumor-infiltrating macrophages is heterogeneous and complicated, which is determined by the cancer type, malignancy development stage, tumor size, as well as location of cancer cells in malignant tissues [152]. Future studies will reveal the effects of ES-exo-GM-CSF vaccination on the identity and heterogeneity of macrophages in lung tumor metastasis.

It has been demonstrated that Th1 and Th17 subsets of $\mathrm{CD}^{+} \mathrm{T}$ cells are involved in lung malignancy and metastasis [141, 142]. Th1 cells increase cellmediated immune responses by producing effector cytokines, including IFN- $\gamma$. Th1 immunity contributes to activation of $\mathrm{CD}^{+} \mathrm{T}$ cells and IFN- $\gamma$ expression in $\mathrm{CD}^{+} \mathrm{T}$ cells [153]. As a subset of pro-inflammatory $\mathrm{T}$ helper cells, Th17 cells are defined by their production of IL-17. The signals from Th17 cells are related to inhibition of Treg differentiation [154]. In our study, there was no significant change in the presence of Th1 and Th17 cells in pulmonary tumor infiltrates 
between mice immunized with ES-exo-GM-CSF and their non-vaccinated counterparts (Figure 3.6), suggesting that this vaccination strategy elicits immunity through mechanisms independent of Th1 and Th17.

$\mathrm{CD}^{+}$cytotoxic $\mathrm{T}$ cells are known to induce anti-tumor immunity by producing cytolytic cytokines, such as IFN- $\gamma$ and TNF- $\alpha$ [155]. However, cytokines released from tumor-infiltrating $\mathrm{CD} 8^{+} \mathrm{T}$ cells are one of the crucial contributing factors in immune response against cancer cells [155]. ESexo/GM-CSF vaccination significantly boosted the frequency of IFN- $\gamma$ producing $\mathrm{CD}^{+}$cytotoxic $\mathrm{T}$ cells in lung metastases (Figure 3.7 ), which is consistent with the data acquired from subcutaneously injected lung tumors (Figure 2.11). However, the levels of TNF- $\alpha$-producing CD8 ${ }^{+}$cytotoxic T cells in metastasized pulmonary malignancies were unaffected by ES-exo/GM-CSF vaccination. In contrast, the levels of TNF- $\alpha$-generating $\mathrm{CD} 8^{+}$cytotoxic $\mathrm{T}$ cells in subcutaneously implanted lung tumors were markedly increased by ESexo/GM-CSF vaccination, suggesting that different tumor microenvironments might determine the production of cytokines by $\mathrm{CD}^{+}$cytotoxic $\mathrm{T}$ cells.

In our studies, when tumor-infiltrating immune cells were acquired for immunoanalysis, tumor metastases in the lungs of mice vaccinated with ESexo-GM-CSF were much smaller than those of non-vaccinated mice (Figure 3.1). The observed difference in intratumoral lymphocyte profiles between vaccinated group and control group could be partially attributed to various stages of lung metastases. More experiments are warranted to examine tumor- 
infiltrating immune cells from lung metastases at similar stages in the future.

In summary, the protection against metastasized lung cancer afforded by ES-exo/GM-CSF vaccination involves a number of immune response pathways.

Future studies will focus on elucidating how this vaccination strategy not only promotes the effector function of the immune system but also suppresses various immune evasion mechanisms conferred by developing lung metastases, which is attributed to the robust efficacy against metastasized pulmonary malignancies. 
CHAPTER IV

THE PLURIPOTENCY OF EMBRYONIC STEM CELLS IS ESSENTIAL FOR CANCER-PREVENTION EFFICACY OF EMBRYONIC STEM CELL-BASED VACCINE

\section{INTRODUCTION}

The association between embryonic materials and tumors has initiated many research activities on exploring the therapeutic potential of embryonic stem cells (ESCs) against cancer [26, 29]. In the 1960s, Triolo proposed a theory that cancer originates from ESCs [84], indicating that cancer cells possess similar characteristics as ESCs. It is well known that ESCs and tumor cells share some antigens. Since 1960s, the antigen overlap between embryonic tissues and neoplastic cells, such as carcinoembryonic antigen (CEA), prostate-specific antigen (PSA), and cancer/testis antigen (CTA), has been extensively studied, [18]. However, the full extent to which embryonic/cancer tissues overlap in antigens remains unknown. Thus, understanding this overlap remains an area 
of ongoing research. It has been demonstrated that embryonic materials possess immune regulatory activity to prevent cancers as a vaccination strategy [26]. Exploiting the antigenic similarity between malignant cells and ESCs, a recent study reports the development of an anti-lung cancer vaccine based on allogeneic murine ESCs along with allogeneic murine fibroblasts expressing granulocyte-macrophage colony stimulating factor (GM-CSF) as an immunostimulatory adjuvant [26]. Another study provides support for this strategy by demonstrating that irradiated, induced pluripotent stem cells (iPSCs) function as a prophylactic vaccine against transplanted lung tumors [30]. Nevertheless, further research is needed to completely elucidate how the similarity between cancer cells and ESCs contributes to the immunity of ESCs against cancer.

The pluripotency of ESCs is one of the most specialized properties shared with cancer stem cells [156]. Both tumors and stem cells are capable of selfrenewal and phenotypic plasticity[156]. The prognosis of human malignancies often depends on the differentiation status of the tumor type with the worst prognosis usually induced by poorly differentiated tumors. A panel of genes identified in different histologically poorly differentiated tumors are normally enriched in ES cells [85]. Their results indicate that genes encoding specific transcriptional regulators in ESCs are often overexpressed in poorly differentiated tumors. This suggests that the preventative effect of ESCs against cancer is likely attributed to their pluripotency. 
It appears that the mechanisms involved in the prophylactic efficacy of vaccines derived from diverse early stage embryonic cells vary to a certain degree. When immunized with a vaccine consisting of murine ESCs along with GM-CSF, mice display robust tumor-reactive primary and memory $\mathrm{CD}^{+} \mathrm{T}$ effector responses against lung malignancy [29]. In contrast, anti-cancer immunity of an iPSC-based vaccine is associated with reduction of a subset of pro-inflammatory $\mathrm{T}$ helper cells Th17 and increased $\mathrm{CD} 11 \mathrm{~b}^{+} \mathrm{GR} 1^{\text {hi }}$ myeloidderived suppressor cells [30].

Immunization with antibodies specific for tumor-associated antigens has been reported to trigger an immunological response against tumor cells through antibody-dependent cellular cytotoxicity (ADCC), a cell-mediated immune defense mechanism [157-159]. In this process, membrane-surface antigens of cancer cells are recognized by specific antibodies, leading to the interaction between antibodies and effector immune cells as well as subsequent the lysis of tumor cells. Since ADCC relies on a preceding antibody response to be effective, it is part of the adaptive immune response. Although natural killer (NK) cells are known to be the typically effector cells to eliminate neoplastic cells during ADCC, macrophages, neutrophils and eosinophils are also capable of mediating ADCC against cancer [160-163]. Currently, the cellular populations responsible for ADCC in the context of ESC-derived tumor vaccines is unknown. Therefore, further research is needed to determine the involvement of ADCC in the prophylactic efficacy of ESC-derived anti-tumor vaccines. 
In this study, we explored the importance of pluripotency of ESCs in their prophylactic efficacy against lung cancer. We also investigated the antigens specific for the antibodies generated by ESC-based vaccines. 


\section{MATERIALS AND METHODS}

\section{Cell lines}

Murine embryonic stem cell line ES-D3 (CRL-11632) and murine Lewis lung carcinoma (CRL-1642) were acquired from ATCC (Manassas, VA). Murine fibroblast cell line STO (ATCC \# CRL-1503) infected with a replication-defective retrovirus expressing murine granulocyte-macrophage colony-stimulating factor (GM-CSF) cDNA was acquired from Dr. Glenn Dranoff (Novartis Institutes for Biomedical Research; Boston, MA). ES-D3 cells and Lewis lung carcinoma (LLC) were cultured as described in Chapter 2. STO cells were grown and maintained in Dulbecco's modified eagle's medium (SH30243.LS; GE Life Sciences; Pittsburgh, PA) supplemented with 10\% fetal bovine serum (900-108; Gemini; Broderick, CA), 100 units/ml penicillin (sc45000-652; Mediatech; Manassas, VA) and $100 \mu \mathrm{g} / \mathrm{ml}$ streptomycin (sc45000-652; Mediatech). GMCSF produced by STO cells was evaluated by measuring GM-CSF concentration in the culture medium with a murine GM-CSF ELISA kit (88733422; Thermo Fisher; Waltham, MA) following manufacturer's protocol. All of the cells were cultured in a $5 \% \mathrm{CO}_{2}$ humidified incubator at $37^{\circ} \mathrm{C}$.

\section{Mice}

Wild-type male and female C57BL/6 mice (8 weeks of age) were acquired from Jackson Laboratory (Bar Harbor, ME). Mice were maintained at the University of Louisville Research Resources Facilities (RRF). The facility is accredited by the American Association for the Accreditation of Laboratory Animal Care (AAALC), and adheres to the "Guide for the Care and Use of Laboratory 
Animals" (Institute of Laboratory Animal Resources, National Research Council, National Academy Press, 1996). The Institutional Animal Care and Use Committee (IACUC) at the University of Louisville has approved the mouse study (protocol number: 18301).

\section{Differentiation of murine ESCs}

Murine ESCs were differentiated into neuronal cells following a published protocol [164]. Briefly, ES-D3 cells were seeded onto a standard 10-cm petri dish in standard ESC media lacking leukemia inhibitory factor (LIF) and $\beta$ mercaptoethanol. Two days later, the media was replaced with fresh medium, and the cells were cultured for 2 additional days. Next, media was replaced with fresh media containing $0.5 \mu \mathrm{M}$ retinoic acid (R2625; Sigma-Aldrich; St. Louis, MO) and the cells were cultured for 2 days. Finally, the cells were cultured in fresh retinoic acid-containing media for another 2 days. The morphology of differentiated cells was examined using an EVOS cell imaging system (Thermo Fisher). The efficacy of differentiation was evaluated by examining the pluripotency of differentiated cells with measurement of SSEA-1, SSEA-4 and Oct-3/4 expression levels by flow cytometry as described in Chapter 2.

\section{Anchorage-independent cell culturing}

After heating in a $42^{\circ} \mathrm{C}$ water bath for 30 minutes, $3.5 \%$ soft agar solution was diluted to $0.7 \%$ with fresh ES-D3 cell culture medium. To form the agar base, 3 $\mathrm{ml}$ of $0.7 \%$ agar solution was plated in each well of a 6 -well tissue culture plate. The agar in the plates was then solidified at $4^{\circ} \mathrm{C}$ for 30 minutes. Parental ESD3 cells, differentiated ES-D3 cells, and Lewis lung carcinoma (LLC) cells ( $3 x$ $10^{3}$ or $7.5 \times 10^{3}$ ) were transferred on top of agar base in each well in a final 
volume of $3 \mathrm{ml} 0.7 \%$ agar solution. Each cell line and seeding density was plated in duplicate. The plates were placed at $4^{\circ} \mathrm{C}$ for 5 minutes before being transferred to a $5 \% \mathrm{CO}_{2}$ humidified incubator at $37^{\circ} \mathrm{C}$ for 8 day-culture. The plates were then stained with $1 \mathrm{mg} / \mathrm{ml}$ iodonitrotetrazolium chloride (I10406; Sigma-Aldrich) overnight. The images were acquired using an EVOS cell imaging system and the numbers of cell colonies were determined using the software ImageJ (National Institutes of Health; Bethesda, MD).

\section{Vaccination against tumor challenge}

Parental or differentiated ESCs and STO cells overexpressing GM-CSF were disassociated from tissue culture plates with $0.05 \%$ trypsin/EDTA (25-052-Cl; Corning; Corning, NY). Collected cells were washed twice with sterile 1 x PBS and suspended in $1 \times$ PBS at a concentration of $10 \times 10^{6} / \mathrm{ml}$. Parental or differentiated ESCs $\left(1 \times 10^{6}\right)$ along with GM-CSF-expressing STO cells $\left(1 \times 10^{6}\right)$ were injected subcutaneously (s.c.) in the right flank of 8-week old male or female C57BL/6 mice. Primary vaccination was carried out on day 0 , and a boost vaccination was administered on day 7 . Next, 7 days after the second vaccination, vaccinated mice were subcutaneously administered with LLC cells $\left(0.15 \times 10^{6}\right)$ on the left flank. Side effects of tumor development in mice were monitored daily, and the resultant tumors were evaluated using dull-edged Vernier calipers. Tumor growth was monitored by measuring tumor width (W) and length $(\mathrm{L})$ in $\mathrm{mm}$ using calipers every other day and tumor volumes $(\mathrm{V})$ in $\mathrm{mm}^{3}$ were calculated by the following formula: $V=(W * W * L) / 2$. Mice were 
euthanized when tumors exceeded $5 \%$ of body weight. The remaining mice were tumor-free with no overt signs of distress. On day 72 , tumor-free mice were challenged with LLC cells $\left(0.15 \times 10^{6}\right)$ for second time by subcutaneous injection on the left flank. Tumor growth was monitored daily.

\section{Proteomics analysis of exosome samples}

Exosomes isolated from ES-D3 cells were extracted with lysis buffer containing $50 \mathrm{mM}$ Tris (pH7.4), $150 \mathrm{mM} \mathrm{NaCl}, 1 \% \mathrm{NP}-40$, and $0.05 \%$ SDS and stored at $4^{\circ} \mathrm{C}$. Exosome lysate was analyzed at the Genome Center, University of California at Davis. Following the standard procedure of protein digestion by Trypsin/Lys-C (V5071; Promega; Madison, WI), exosomes were desalted by C18 Microspin columns (SEMSS18V; Nest Group; Southborough, MA) and lyophilized by vacuum centrifugation. Liquid chromatography (LC) separation was carried out with an Easy-nLC 1000 LC system (Thermo Fisher Scientific). Then digested peptides were reconstituted in $2 \%$ acetonitrile/0.1\% trifluoroacetic acid. The samples $(3 \mu \mathrm{g})$ were loaded onto a 100 micron $\times 25$ mm Magic C18 $100 \AA 5 \mathrm{U}$ reverse phase trap where they were desalted online

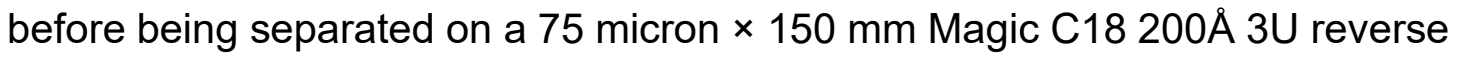
phase column. Peptides were eluted with a gradient of $0.1 \%$ formic acid $(A)$ and $100 \%$ acetonitrile (B), which was run with $5 \%$ to $35 \%$ B (45 minutes), $35 \%$ to $80 \%$ B (8 minutes), $80 \%$ B (1 minute), $80 \%$ to $5 \%$ B (1 minute), and $5 \%$ B (10 minutes). The collection of mass spectra (MS) was carried out with a mass spectrometer (Orbitrap Q Exactive Plus; Thermo Fisher Scientific) in a data- 
dependent mode with MS precursor scan followed by 15 MS/MS scans. A dynamic exclusion of $15 \mathrm{~s}$ was used. All MS/MS samples were analyzed using X! Tandem (CYCLONE; 2013.02.01.1) to search the uniprot mouse proteome plus an equal number of reverse decoy sequences (142010 entries). MS/MSbased peptide and protein identifications were validated with the software Scaffold (Scaffold_4.8.2; Proteome Software; Portland, OR). Peptide identifications were accepted if they could be established at greater than $98.0 \%$ probability by the Scaffold Local FDR algorithm. Protein identifications were accepted if they could be established at greater than $5.0 \%$ probability to achieve an FDR less than $5.0 \%$ and contained at least one identified peptide.

\section{Antigen capture experiments for mice immunized with exosomes.}

Male C57BL/6 mice (8 weeks of age, 2 mice/group) were immunized twice (day 0 and day 7 ) with vehicle only (1x PBS) or $225 \mu \mathrm{g}$ of exosomes isolated from ES-D3 cells stably-expressing GM-CSF by subcutaneous injection. Seven days following the second vaccination, whole blood was collected by cardiac puncture immediately after $\mathrm{CO}_{2}$ asphyxiation. Whole blood was transferred to serum separator tubes (365967; Beckon Dickinson; Franklin Lakes, NJ) and serum was collected. Protein A agarose beads (200 $\mu$ l; 9863S; Cell Signaling; Danvers, MA) were resuspended with $1 \mathrm{ml}$ binding buffer containing $50 \mathrm{mM}$ Tris $(\mathrm{pH} 7.5)$ and $150 \mathrm{mM} \mathrm{NaCl}$ and centrifuged at $390 \times \mathrm{g}$ for 30 seconds. Binding buffer was removed and wash was repeated twice. The mouse serum collected from 2 mice was combined ( $200 \mu \mathrm{l}, 100 \mu \mathrm{l}$ from each mouse) and added to the 
tubes containing the beads. The mixture was incubated on a rotator for 3 hours at room temperature.

Exosomes isolated from ES-D3 cells expressing GM-CSF $(9 \mathrm{mg})$ were resuspended in $1.2 \mathrm{ml} 2 \mathrm{X}$ lysis buffer containing $100 \mathrm{mM}$ Tris (pH7.5), $300 \mathrm{mM}$ $\mathrm{NaCl}, 2 \% \mathrm{NP}-40,0.05 \%$ SDS, protease inhibitor (P8349; Sigma-Aldrich) and phosphatase inhibitor (04 906845 001; Roche Diagnostics; Indianapolis, IN). The lysate of exosomes was first sonicated with $10 \%$ amplitude for 5 seconds using a Sonic Dismembrator (Branson Digital Sonifier, Thermo Fisher). The exosomal lysate of was then passed through an insulin syringe 10 times and centrifuged at $13,000 \times \mathrm{g}$ for 10 minutes at $4^{\circ} \mathrm{C}$. The supernatant was taken out as exosomal extract for the antigen binding experiment. The serum-bead mixtures were centrifuged at $390 \times \mathrm{g}$ for 30 seconds at $4^{\circ} \mathrm{C}$. The beads were washed for 3 times with $500 \mu$ l of lysis buffer by rotating for 10 minutes at $4^{\circ} \mathrm{C}$. The exosomal extract $(1 \mathrm{ml}$ ) was added to each tube containing the beads, and the tubes were rotated at $4^{\circ} \mathrm{C}$ overnight. Then the extract-bead mixtures were centrifuged at $390 \times \mathrm{g}$ for 30 seconds at $4^{\circ} \mathrm{C}$. The beads were washed 5 times with $1 \mathrm{ml}$ of binding buffer by rotating for 10 minutes at $4^{\circ} \mathrm{C}$. Abundance of proteins bound to the beads was evaluated by proteomic analysis at the Genome Center, University of California at Davis.

\section{Antigen capture experiments for mice immunized with ESCs.}

Vehicle only $(1 \times$ PBS $)$ or ES-D3 cells $\left(1 \times 10^{6}\right)$ combined with STO-fibroblasts 
expressing GM-CSF $\left(1 \times 10^{6}\right)$ were subcutaneously injected at right flank into eight-week old male C57BL/6 mice (2 mice/group) twice with one week apart. One week following the second immunization, serum was collected as described previously. Collected serum was incubated with protein A agarose beads in the same fashion as the serum acquired from mice immunized with exosomes as described above.

ES-D3 cells were collected ( $30 \times 10^{6}$ cells/each group) by trypsinization. Cell pellets were resuspended in $5 \mathrm{ml}$ lysis buffer and sonicated at $20 \%$ amplitude (Branson Digital Sonifier) twice for 5 seconds each time with at least one minute-rest on ice between each 5-second pulse. Then the cell lysate was pushed through an insulin syringe 10 times and centrifuged in a microcentrifuge at $13,000 \times \mathrm{g}$ for 10 minutes at $4^{\circ} \mathrm{C}$. The supernatant was taken out as whole cell extract for the antigen binding experiment. Whole cell extract was then added to protein A agarose beads pre-incubated with the serum as described above for the serum from exosome-vaccinated mice. Samples were then examined by proteomics analysis.

\section{Proteomics analysis of protein samples bound to beads}

Protein samples bound to protein A agarose beads were washed four times with $200 \mathrm{ml}$ of $50 \mathrm{mM}$ ammonium bicarbonate (AMBIC; A6141; Sigma-Aldrich) with a 20-minute shake at $4^{\circ} \mathrm{C}$ between each wash. About $2.5 \mu \mathrm{g}$ of Trypsin/Lys-C (V5071; Promega) was added to the beads and AMBIC mixture and the 
samples were digested overnight at 800 x rpm shake speed. Following digestion, the supernatant was transferred to a microfuge tube and the beads were washed once with $50 \mathrm{mM} \mathrm{AMBIC}$. After a 20-minute gentle shake, the wash was transferred and combined with the initial supernatant. The peptide extracts were reduced in volume by vacuum centrifugation and a small portion of the extract was used for fluorometric peptide quantification (23290; Thermo Fisher Scientific). One microgram of sample based on the fluorometric peptide assay was loaded for each LC-MS analysis.

Digested peptides were analyzed by LC-MS/MS on a Thermo Scientific $Q$ Exactive Orbitrap Mass spectrometer in conjunction Proxeon Easy-nLC II HPLC (Thermo Fisher Scientific) and Proxeon nanospray source. The digested peptides were loaded a 100 micron x 25 mm Magic C18 100A 5 U reverse phase trap where they were desalted online before being separated using a 75 micron x 150 mm Magic C18 200A 3U reverse phase column. Peptides were eluted using a 60-minute gradient with a flow rate of $300 \mathrm{nl} / \mathrm{min}$. An MS survey scan was obtained for the $\mathrm{m} / \mathrm{z}$ range $300-1600, \mathrm{MS} / \mathrm{MS}$ spectra were acquired using a top 15 method, where the top 15 ions in the MS spectra were subjected to HCD (High Energy Collisional Dissociation). An isolation mass window of 2.0 $\mathrm{m} / \mathrm{z}$ was for the precursor ion selection, and normalized collision energy of $27 \%$ was used for fragmentation. A 15-second duration was used for the dynamic exclusion.

Tandem mass spectra were extracted and charge state was deconvoluted 
by Proteome Discoverer (Thermo Fisher Scientific). MS/MS samples were analyzed using X! Tandem (The GPM, thegpm.org; version TORNADO (2013.02.01.1)). X! Tandem was set up to search (20180405 Uniprot Mouse) database (108924 entries), the CRAP database of common laboratory contaminants (www.thegpm.org/crap; 114 entries) plus an equal number of reverse protein sequences assuming the digestion enzyme trypsin. X! Tandem was searched with a fragment ion mass tolerance of 20 PPM and a parent ion tolerance of 20 PPM. Deamidation of asparagine and glutamine, oxidation of methionine and tryptophan, sulphone of methionine, tryptophan oxidation to formylkynurenin of tryptophan and acetylation of the n-terminus were specified in $\mathrm{X}$ ! Tandem as variable modifications.

Scaffold (version 4.4.1, Proteome Software) was used to validate MS/MSbased peptide and protein identifications. Peptide identifications were accepted if they could be established at greater than $85.0 \%$ probability by the Scaffold Local FDR algorithm. Protein identifications were accepted if they could be established at greater than $80.0 \%$ probability to achieve an FDR less than $5.0 \%$ and contained at least 1 identified peptide. Protein probabilities were assigned by the Protein Prophet algorithm (reference). Proteins that contained similar peptides and could not be differentiated based on MS/MS analysis alone were grouped to satisfy the principles of parsimony. Proteins sharing significant peptide evidence were grouped into clusters. 
10. Statistical analysis

All of the experiments were performed in replicates. The statistical analysis was carried out by the student t-test and the analysis of variance (ANOVA). AP value $<0.05$ was considered significant. 


\section{RESULTS}

\section{The pluripotency of murine embryonic stem cells (ESCs) is reduced during differentiation}

ESCs and lung cancer cells express common protein markers and share several genotypic and phenotypic traits $[11,18,165]$. Among them, pluripotency is one of the most specialized properties shared between ESCs and a subset of lung tumor cells, cancer stem cells (CSCs). We reasoned that the anti-lung tumor immunity generated by ESC-based vaccine, either as intact cells or as secreted exosomes, is likely attributed to shared carcinoembryonic antigens whose expression is reduced or eliminated during differentiation. A published approach was employed to differentiate murine ESC line ES-D3, in which deprivation of extrinsic self-renewal signals from leukemia inhibitory factor (LIF) coupled with the addition of retinoic acid drove undifferentiated cells to become neuronal cells [164, 166]. Morphological characterization of parental and differentiated ES-D3 cells was carried out using microscopy (Figure 4.1A). Undifferentiated parental ES-D3 cells had morphology of typical ESCs with rounded cell shape and smooth cytoplasmic membrane, and they generated close cytoplasmic membrane contact with each other to form colonies [167]. In contrast, differentiated ES-D3 cells displayed different morphology with dendrite-like protrusions, which is consistent with the morphological characteristics of neuronal cells [168]. 
A number of molecular markers have been reported to be indicative of the pluripotency of murine ESCs, among which SSEA-1, SSEA-4, and Oct-3/4 expression are commonly studied to examine the pluripotency status [169]. Generally, pluripotent murine ESCs exhibit elevated SSEA-1 and Oct-3/4 reactivity, but low expression of SSEA-4 [170]. Therefore, the pluripotency of differentiated ES-D3 cells was evaluated by measuring SSEA-1, SSEA-4 and Oct-3/4 expression levels via flow cytometry as described earlier (Figure 2.3). Loss of pluripotency of differentiated ES-D3 cells was indicated by decreased SSEA-1 and Oct-3/4 expression and increased SSEA-4 expression compared with parental ES-D3 cells (Figure 4.1B). Overall, differentiated ES-D3 cells are distinct from their undifferentiated counterparts at both morphological and molecular levels. 
A

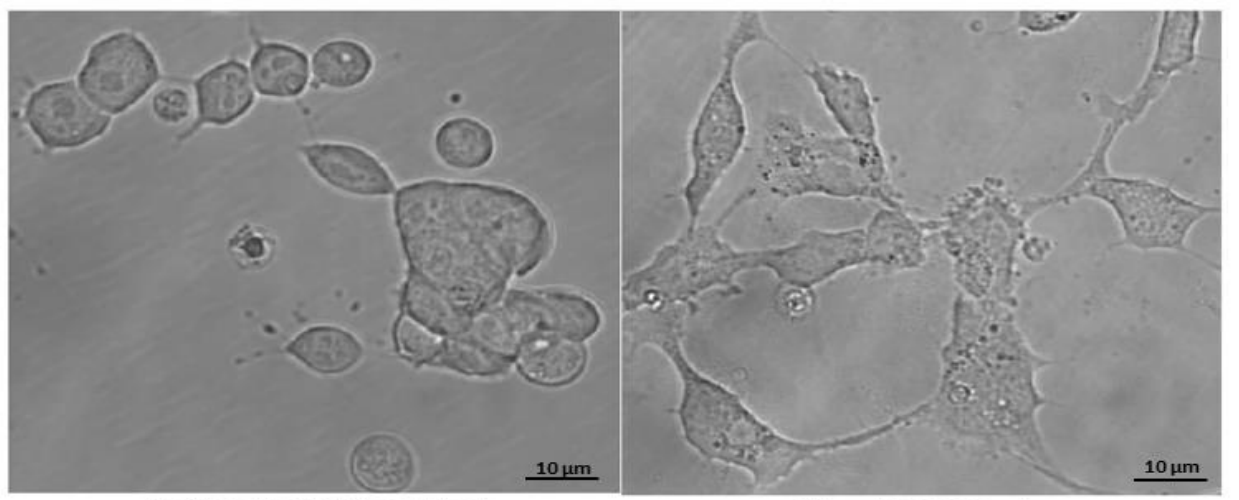

Undifferentiated ESD3 parental cells

Differentiated ESD3 cells

B

Undifferentiated

Differentiated
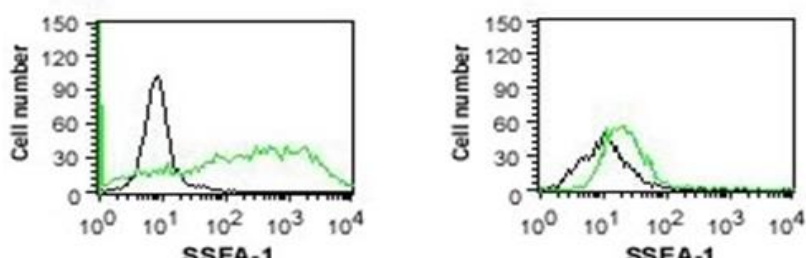

isotype antibody contro

anti-SSEA-1 antibody
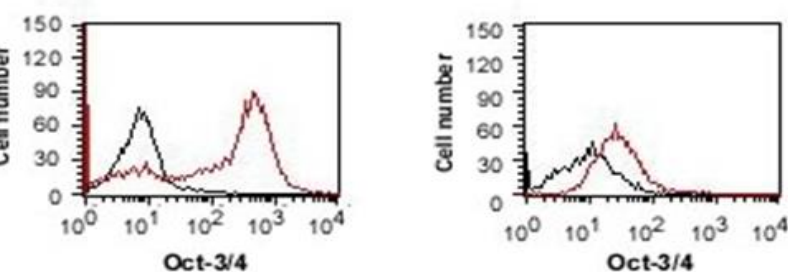

isotype antibody control anti-Oct-3/4 antibody
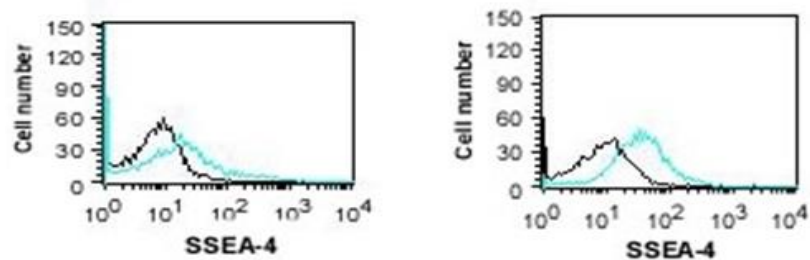

sotype antibody control anti-SSEA-4 antibody 
Figure 4.1. Differentiated ES-D3 cells are characterized.

Murine embryonic stem cell line ES-D3 cells were cultured in the absence of LIF and $\beta$-mercaptoethanol for 4 days. Then ES-D3 cells were cultured in the presence of $0.5 \mathrm{mM}$ retinoic acid for 4 days. (A) Representative images of parental and differentiated ES-D3 cells. Scale bar, $10 \mu \mathrm{M}$. (B) Flow cytometry analysis of the expression of pluripotency and differentiation markers (SSEA-1, Oct3/4 and SSEA-4) in parental and differentiated ES-D3 cells. 


\section{The pluripotency of ESCs is important for their anchorage-independent growth potential}

A hallmark of tumorigenesis is the proliferation of neoplastic cells without a solid surface, aka anchorage-independent growth, which correlates with tumorigenic and metastatic potential in vivo [171]. The soft agar colony formation assay is a well-accepted method to evaluate anchorage-independent growth [172]. Due to anoikis, a particular type of apoptosis, no colonies are formed when normal cells are plated in an anchorage-independent culture model [173]. Although ESCs and malignant cells share some genotypic and phenotypic traits, it was unclear whether ESCs possess anchorage-independent growth potential, as tumor cells do. Equal numbers of parental ES-D3 cells and Lewis lung carcinoma (LLC), an aggressive non-small cell lung cancer cell line, were seeded in soft agar. As shown in Figure 4.2, LLC cells and parental ES-D3 cells showed similar anchorage-independent growth capacity. To our knowledge, this is the first study demonstrating that the anchorage-independent growth potential of undifferentiated ESCs is the same as that of lung tumor cells. To further explore the involvement of pluripotency of ESCs in anchorageindependent cell growth, we examined the ability to form colonies in soft agar of differentiated ES-D3 cells, which was reduced to extremely low levels compared with those of undifferentiated ES-D3 and LLC cells. These results indicate that pluripotency of ESCs is critical for their ability to evade anoikis and grow independent of a solid surface. 
A

Seeded cells (x 10^3)

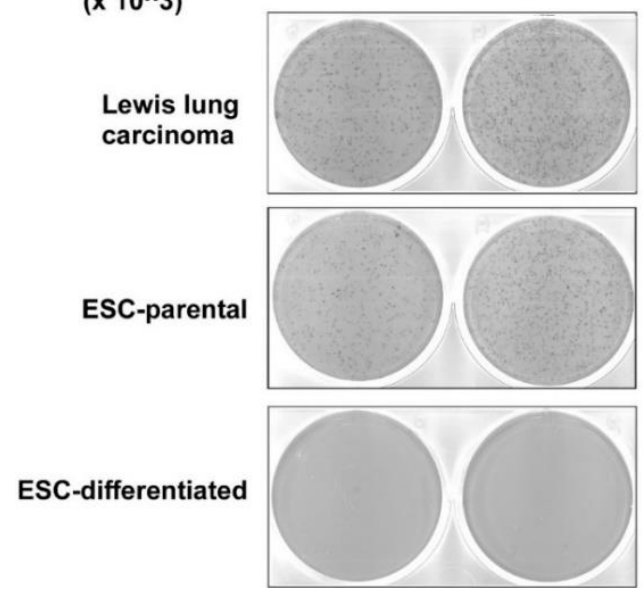

B

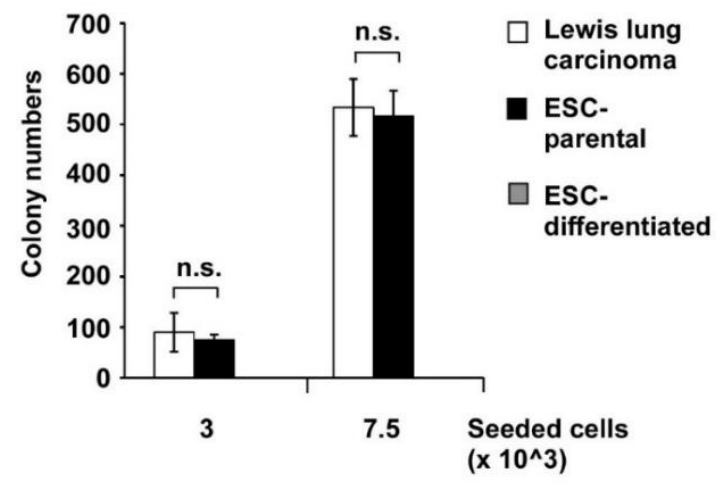

Figure 4.2. Differentiation inhibits anchorage-independent proliferation of ES-D3 cells.

The anchorage-independent growth capability of differentiated ES-D3 cells and their parental counterparts was examined by a soft agar colony formation assay. (A) The indicated number of LLC, parental ES-D3 cells and differentiated ESD3 cells were cultured in soft agar for 8 days. Representative images of the plates are shown. (B) The colonies of LLC, parental ES-D3 and differentiated ES-D3 on the soft agar plates shown in (A) were counted. The data are presented as means \pm standard deviations of three independent experiments. Student's unpaired t test. Asterisks $\left(^{* *}\right)$, indicate $p<0.001$. "ns", no significance. 


\section{Differentiation decreases the efficacy of ESC vaccine on lung tumor development}

Once the reduction in pluripotency of ES-D3 cells by differentiation was confirmed, the role of pluripotency in anti-tumor efficacy of ES-D3 cells was evaluated. To ensure rigor and reproducibility, animal studies were designed to include appropriate control and treatment groups with both sexes. To achieve power and statistical significance, there were 8 animals in each experimental group. Subcutaneous (s.c) injection of vaccines was carried out to induce immunization as described earlier [29]. Three groups of C57BL/6 mice were immunized (aged 8 weeks, 8 per sex per group): vehicle control ( 1 x PBS), undifferentiated ES-D3 and STO fibroblasts expressing GM-CSF (UNDIFF + STO ) or their differentiated counterparts along with GM-CSF-expressing STO cells (DIFF + STO ). Mice were immunized twice (day 0 and day 7 ) followed by a challenge with subcutaneous injection of highly aggressive LLC cells $(0.15 \times$ $\left.10^{6}\right)$ at day 14 . The kinetics of tumor growth were closely monitored by measuring both the longitudinal and transverse diameters of tumors using digital calipers every 2 days as described above. As depicted by Kaplan-Meier survival curves shown in Figure 4.3, all non-vaccinated control animals died within 28 days of tumor cell challenge, whereas $56 \%$ of mice vaccinated with undifferentiated ES-D3 cells survived beyond 38 days. Importantly, only $31 \%$ of animals immunized with differentiated ES-D3 survived for the 28-day period, indicating that differentiation decreases the cancer prevention efficacy of ESCs. 
Furthermore, the results obtained from male mice are very comparable with those from female mice. In the case of tumor development, all control mice developed tumors 14 days after LLC cell administration (Figure 4.4). In contrast, $56 \%(n=16)$ of mice vaccinated with undifferentiated ES-D3 cells were protected from tumor outgrowth by day 32 post-challenge. Moreover, differentiated ES-D3 cells vaccination decreased the efficacy of the immunization against lung cancer by $31 \%$ compared to undifferentiated ES-D3 cells. Tumor growth patterns were similar between both sexes. 
PBS

A

Parental ES-D3/STO-expressing GM-CSF

Differentiated ES-D3/STO-expressing GM-CSF

个 Imm

$1^{\text {st }}$ Boost

$\operatorname{LLC}\left(0.15 \times 10^{6}\right)$

C57BL/6

Day 0

Day 7

Tumor cells

Day 14

B

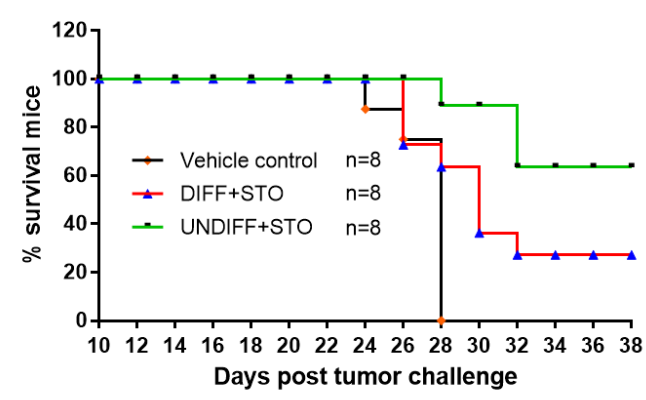

Male
C

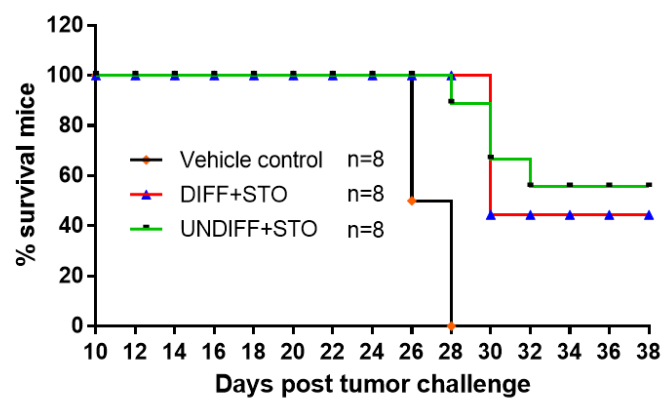

Female

D

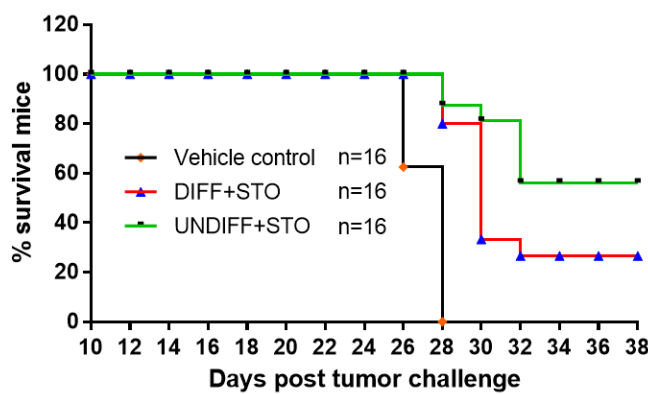

Male + Female 
Figure 4.3. Differentiation decreases the efficacy of ES-D3 cells against lung cancer.

(A) The scheme of immunization is shown. Male and female C57BL/6 mice were immunized twice with $1 \times$ PBS (vehicle control), parental ES-D3/STO expressing GM-CSF (UNDIFF + STO) or differentiated ES-D3/STOexpressing GM-CSF (DIFF + STO) prior to s.c. challenge with LLC cells $(0.15$ $\left.\times 10^{6}\right)$. Tumor growth was monitored daily in all mice until they were euthanized due to tumors exceeding $5 \%$ of body weight. (B) Kaplan-Meier survival curve demonstrates the probability of tumor-free survival in male mice $(n=8)$. (C) Kaplan-Meier survival curve compares the survival of female mice in different vaccination groups $(n=8)$. (D) The data shown in (B) and (C) are combined (16 mice/group). 
A

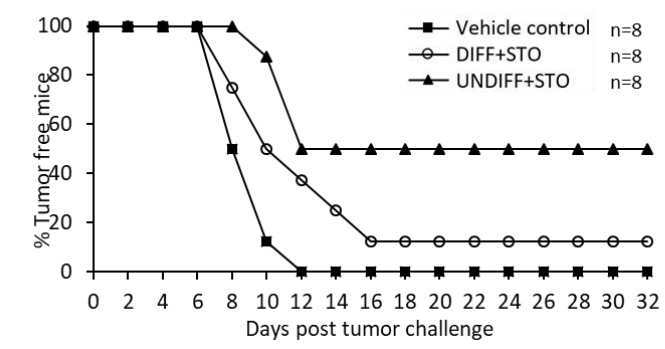

C

Male
B

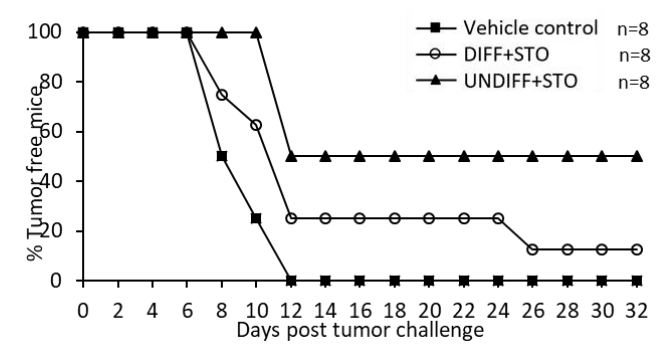

Female

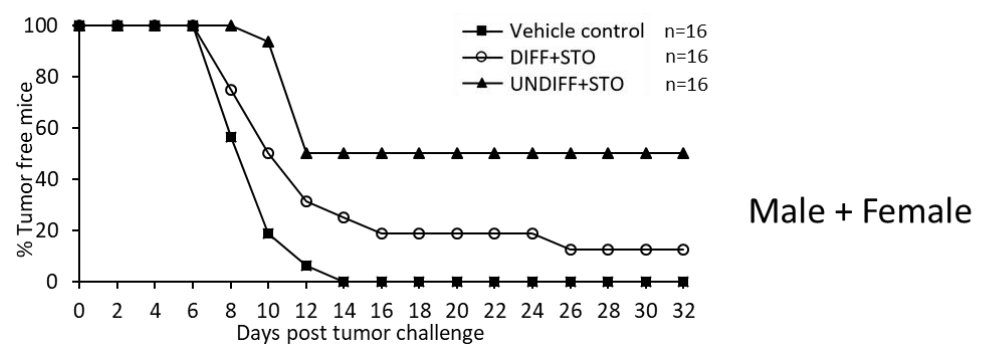

Figure 4.4. Differentiation of ES-D3 cells inhibits their ability to prevent

lung cancer.

C57BL/6 mice were vaccinated twice (days 0 and 7) with 1 x PBS (vehicle control), parental ES-D3/STO expressing GM-CSF (UNDIFF + STO) or differentiated ES-D3/STO expressing GM-CSF (DIFF + STO) followed by s.c. injection of $0.15 \times 10^{6} \mathrm{LLC}$ cells on day 14 . Tumor growth was examined daily. (A) The percentage of tumor-free male mice (8 mice/group) are shown. (B) The data of tumor occurrence in female mice (8 mice /group) are presented (C) Tumor development in both male and female mice is evaluated (16 mice/group, 8 mice/gender). 
More importantly, the growth of LLC tumors that developed in mice vaccinated with undifferentiated ES-D3 cells was slower compared with those of non-vaccinated control mice and mice immunized by differentiated ES-D3 cells (Figure 4.5). These results lend more credence to the notion that the differentiation of ESCs decreases their anti-tumor efficacy in mice.

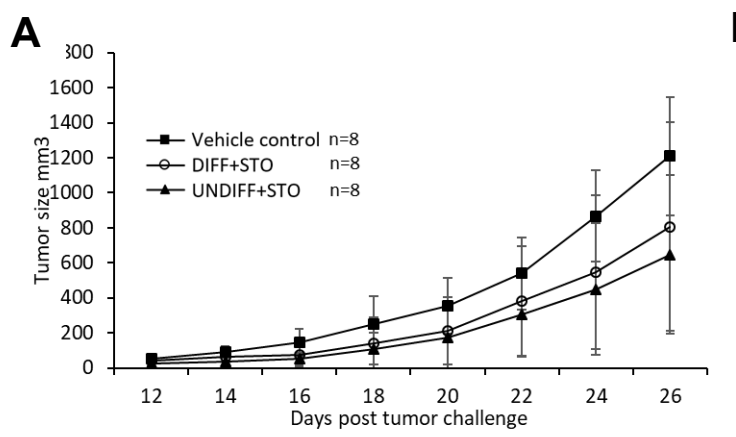

C
B

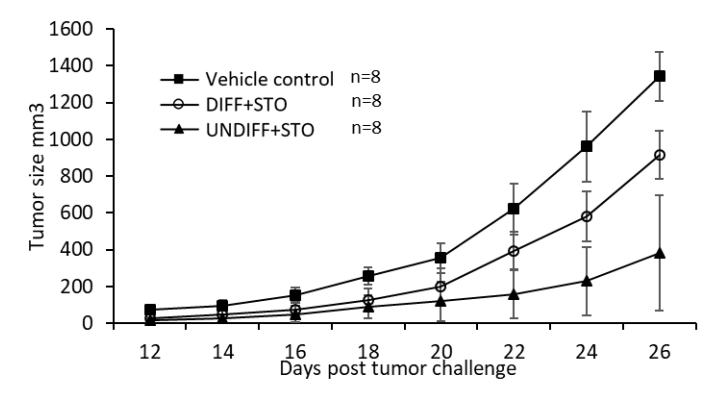

Female

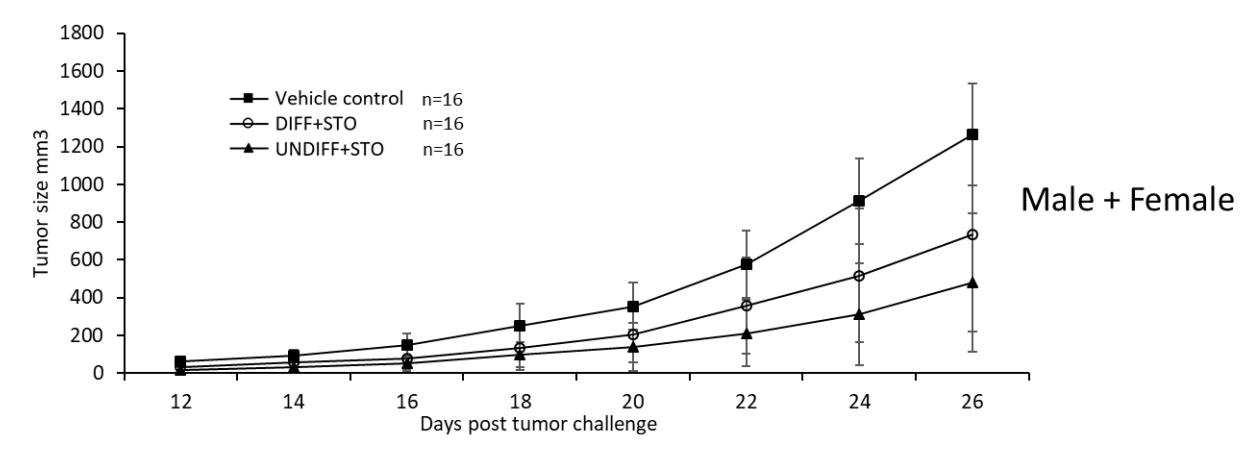

Figure 4.5. Differentiation reduces anti-cancer activities of ES-D3 cells.

C57BL/6 mice were injected twice (days 0 and 7) with $1 \times$ PBS (vehicle control), parental ES-D3/STO expressing GM-CSF (UNDIFF + STO) or differentiated ES-D3/STO expressing GM-CSF (DIFF + STO). On day 14, LLC cells $\left(0.15 \times 10^{6}\right)$ was inoculated into mice by s.c. injection. Tumor growth was determined by dull-edged Vernier calipers every two days. Tumor volumes are presented as means \pm standard deviations. The data represent male mice $(A)$, female mice $(B)$ and both genders $(C)$. 
To evaluate the involvement of ESC pluripotency in the generation of longterm immunity against lung cancer, we tested the anti-tumor responses using mice that had been vaccinated and protected successfully for 58 days. The previously LLC-challenged tumor-free mice were injected with LLC cells for a second time (Figure 4.6A). Notably, 2 tumor-free mice vaccinated with differentiated ES-D3 cells developed tumors 8 days after the second LLC challenge, whereas only $20 \%$ of mice $(n=8)$ in the UNDIFF+STO vaccination group developed tumors following LLC cell re-inoculation (Figure 4.6B). This provides evidence that the pluripotency of ESCs is essential for their long-term anti-tumor activities. 
A

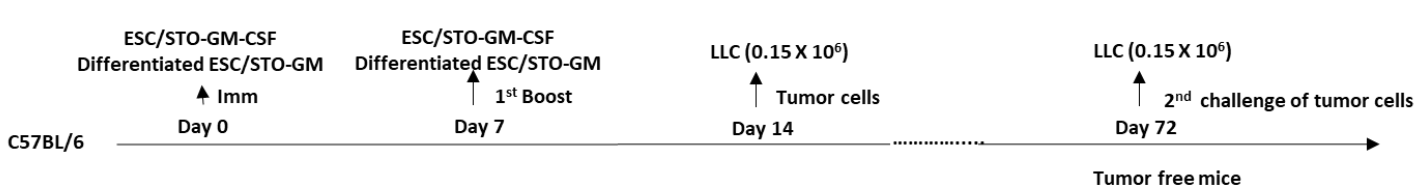

B

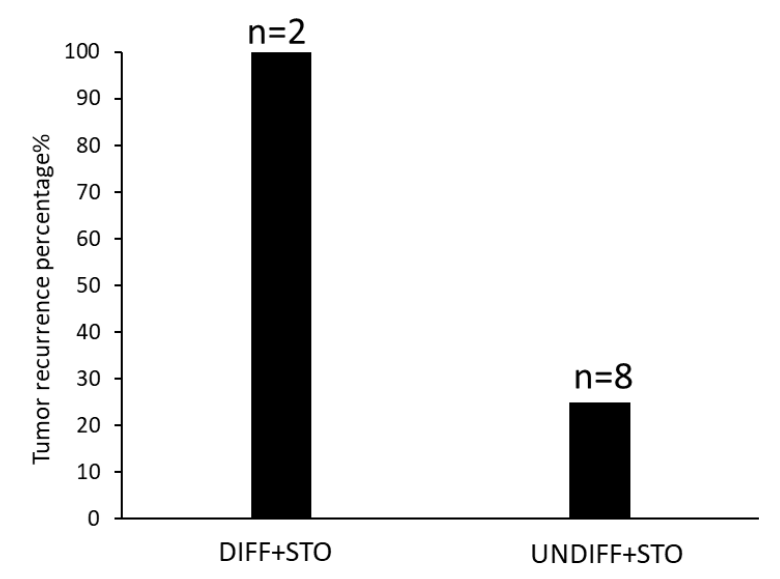

Figure 4.6. Differentiation decreases long-term cancer prevention potential of ES-D3 cells

(A) The scheme of vaccination is shown. tumor-free C57BL/6 mice were immunized with parental ES-D3/STO expressing GM-CSF (UNDIFF + STO) or differentiated ES-D3/STO expressing GM-CSF (DIFF + STO) twice (days 0 and 7), followed by injection inoculation of $0.15 \times 10^{6}$ LLC cells on day 14 . Tumor-free mice were challenged again with LLC cells $\left(0.15 \times 10^{6}\right)$ again at day 72. Tumor development was monitored daily until sacrifice due to tumor sizes reaching the limit ( $5 \%$ of body weight). (B) 28 days after second LLC challenge, the percentages of tumor-free mice in each group were determined. 


\section{ES-D3 cells and lung tumor cells exhibit antigenic similarity as revealed by antigen capture experiments}

There is abundant evidence that malignant cells and ESCs share carcinoembryonic antigens, which are not expressed in normal adult tissues [18]. Those antigens play an important role in cancer vaccination, as they enable the immune system to distinguish tumor cells from nontumor cells, resulting in potential cancer rejection. Since exosomes derived from murine ESCs engineered to produce GM-CSF significantly slowed or blocked the outgrowth of implanted LLC (Figure 2.6), we first examined the presence of proteins in exosomes derived from ES-D3. As shown in Table 4.1, tumor antigens identified in a variety of tumor types, such as alpha fetoprotein (AFP), lactate dehydrogenase (LDH) and fibrinogen, were also present in exosomes derived from ES-D3 cells [174-180], which is in agreement with the notion that embryos and tumors share a group of similar antigens [19, 97, 98]. 


\begin{tabular}{|c|c|c|}
\hline Category & Protein Name & Tumor Type \\
\hline Tumor antigen & Alpha-fetoprotein & $\begin{array}{l}\text { Hepatocellular carcinoma and } \\
\text { germ cell tumors }\end{array}$ \\
\hline Tumor antigen & Lactate dehydrogenase (LDH) & $\begin{array}{l}\text { Germ cell tumor, lymphoma and } \\
\text { leukemia }\end{array}$ \\
\hline Tumor antigen & Fibrinogen & Bladder, ovary, and colon tumors \\
\hline Tumor antigen & Epithelial cell adhesion molecule (EpCAM) / CD326 & Epithelial tumors \\
\hline Tumor antigen & Cluster of differentiation 151 / CD151 & Skin squamous cell carcinoma \\
\hline Tumor antigen & Insulin-like growth factor 2 mRNA-binding & Hepatocellular carcinoma \\
\hline Tumor antigen & Ephrin type-A receptor 2 (EPHA2) & $\begin{array}{l}\text { Breast, prostate, bladder, skin, } \\
\text { lung, ovary, and brain tumors }\end{array}$ \\
\hline
\end{tabular}

Table 4.1 Exosomes derived from ES-D3 cells contains various tumor antigens.

Tumor antigens presented in exosomes isolated from ES-D3 cells were identified by a proteomics approach. 
Since the antigens responsible for prophylactic efficacy of intact ES-D3 cells or exosomes isolated from ES-D3 cells have not been identified yet, we carried out a series of experiments to thoroughly explore this issue. It is possible that the antibodies generated by ESC-based vaccine directly recognize neoplastic cells and initiate antibody-dependent cellular cytotoxicity (ADCC). To gain a comprehensive understanding of the antigenicity of ESC vaccine, a novel immunoproteomic strategy was employed (Figure 4.7). This approach has the potential to reveal the complete repertoire of potentially immunogenic antigens in the serum of immunized mice. This combined affinity chromatography shotgun immunoproteomic approach starts with capture of antibodies in the serum by an IgG affinity column [181]. Specifically, mice (C57BL/6) were immunized with either a cell-based vaccine (ES-D3 cells + STO expressing GMCSF) or an exosome-based vaccine (exosome from GM-CSF-expressing ESD3) as described in "Materials and Methods". After the binding of antibodies to protein A beads, antigen-containing ESC lysates or exosomal lysates were loaded to the respective mixture of protein $A$ beads and antibodies from serum. Bound antigens were identified by liquid chromatography tandem mass spectrometry (LC-MS/MS) analysis. 


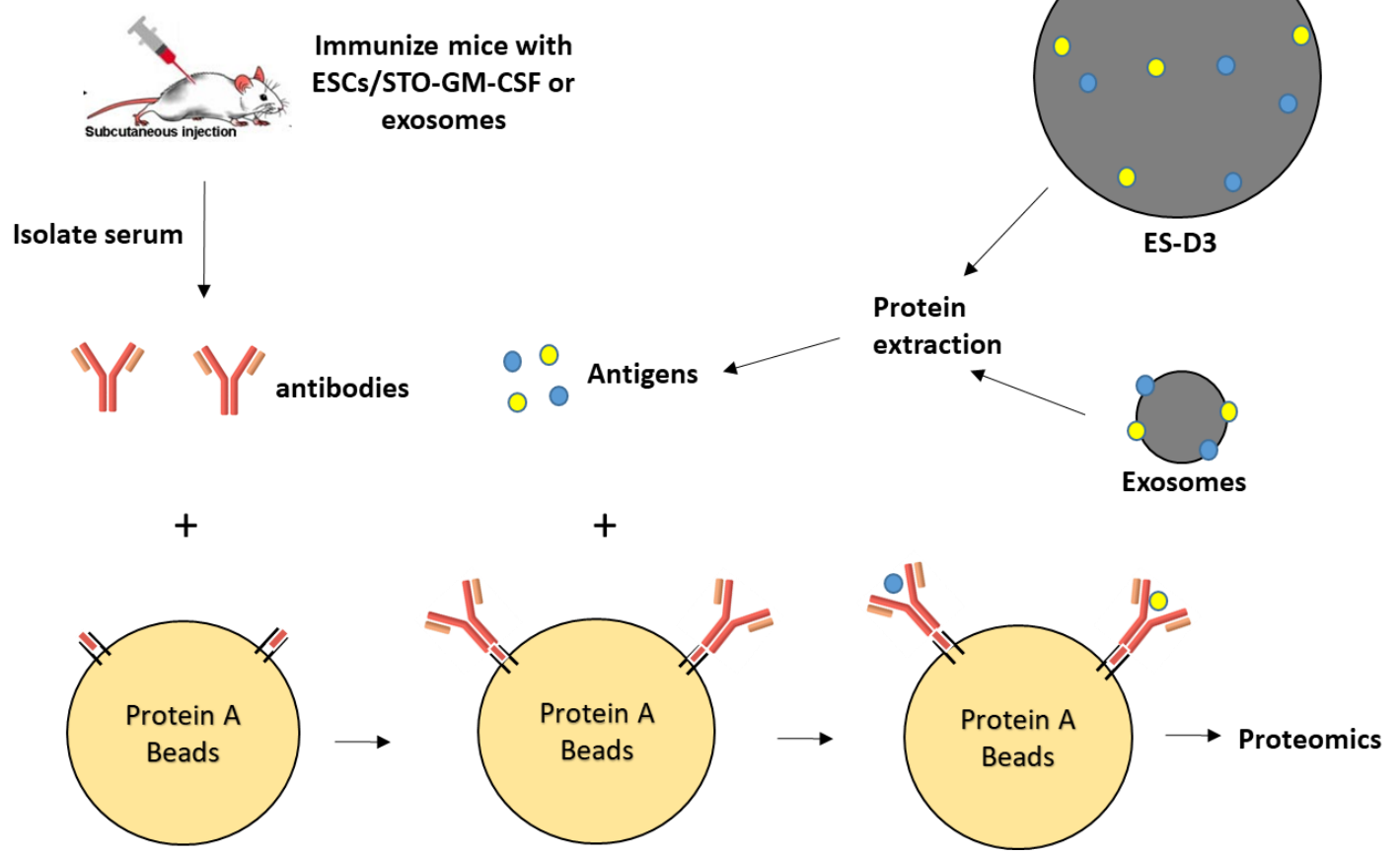

Figure 4.7. An immunoproteomics strategy to identify the antigens specific for the antibodies generated by vaccines derived from ESCs.

The schematic description of a combined affinity chromatography shotgun immunoproteomics approach. C57BL/6 mice were immunized with a vaccine composed of ES-D3 cells and STO fibroblasts expressing GM-CSF) or a vaccine composed of exosomes from GM-CSF expressing ES-D3 cells. Lysates containing antigens of cell- or exosomes-based vaccine were loaded to Protein A agarose beads bound with serum antibodies. LC-MS/MS studies were conducted to identify the antigens bound to the beads 
For the serum acquired from the mice vaccinated with the cell-based vaccine, the presence of 136 immunoglobin-unrelated proteins in the serum of immunized mice is higher than their control counterparts (Figure 4.8A). Among them, 16 identified proteins are members of keratin family and 11 keratin types are cancer related (Table 4.2). Although keratin members are fibrous proteins that form the structural framework of epithelial cells, some of them are commonly used as diagnostic markers for cancers with various epithelial origins, due to their distinctive expression patterns [182]. Importantly, several keratin proteins associated with lung malignancy, keratin $7,8,16,17$, and 19 , were identified in the serum of mice vaccinated with intact ES-D3 cells (Table 4.3).

Similarly, the exosome-based vaccine generated antibodies recognizing 132 non-immunoglobin proteins compared with the vehicle control (Figure 4.8B). Consistent with the results acquired from the cell-based vaccine, a large portion of identified antigens (25 out 132) are keratin members. 10 of which are cancer related (Table 4.2). Furthermore, lung tumor-associated keratin members $8,14,16$, and 17 were also enriched in the serum of exosomeimmunized mice (Table 4.3).

To further evaluate the potential involvement of lung cancer-associated keratin proteins in anti-lung tumor immunity evoked by ESC vaccines, the protein expression profile of LLC cells was examined by a proteomics approach. As expected, several keratins linked to lung tumorigenesis (keratin 7, 8, 16, 17 and 18) were found to express in LLC cells (Table 4.3). These data suggest that 
keratin members $(8,16$, and 17) are candidate antigens responsible for initiating anti-lung tumor immunity through ADCC.

A
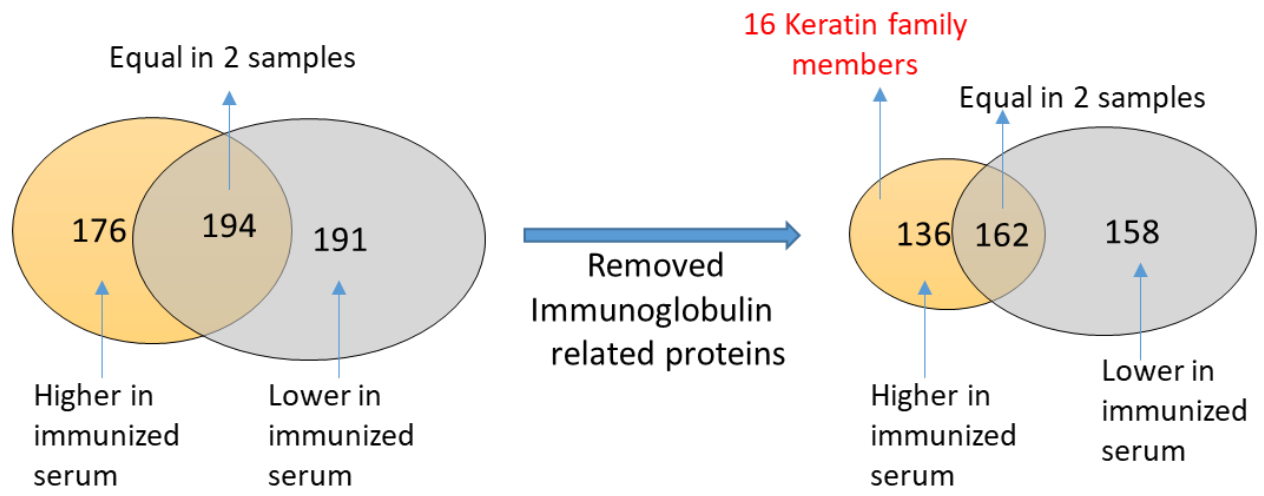

B
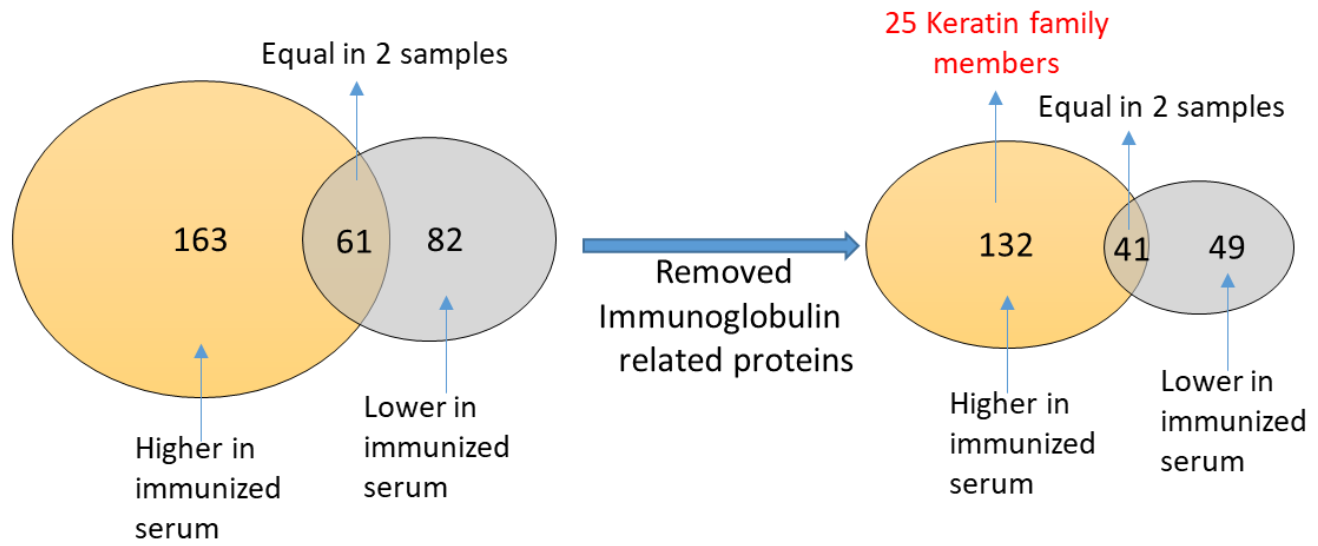

Figure 4.8. Summary of identified antigens specific for vaccinegenerated antibodies.

Antigens were identified by an affinity chromatography shotgun immunoproteomics strategy. (A) Numbers of antigens presented in the serum of mice immunized with cell-based vaccine. (B) Numbers of antigens presented in the serum of mice immunized with exosome-based vaccine. 


\begin{tabular}{|c|c|}
\hline $\begin{array}{c}\text { Keratin } \\
\text { type }\end{array}$ & Correlated tumor type \\
\hline 1 & Skin \\
\hline 4 & Oral \\
\hline 5 & Ovarian \\
\hline 6 & Breast \\
\hline 7 & Lung \\
\hline 8 & Lung, pancreas, colon \\
\hline 14 & Lung, Breast \\
\hline 16 & Lung, cervix, skin \\
\hline 19 & Lung, ovarian, oral \\
\hline 20 & Lung, gastric, pancreas, breast \\
\hline
\end{tabular}

Table 4.2. Keratin members recognized by antibodies in the serum of immunized mice.

Keratin members recognized by antibodies in the serum of mice immunized with cell-based vaccine or exosome-based vaccine are listed. 


\begin{tabular}{|r|r|r|r|}
\hline Keratin type & Cell-based vaccine & $\begin{array}{c}\text { Exosome-based } \\
\text { vaccine }\end{array}$ & LLC \\
\hline 7 & + & - & + \\
\hline 8 & + & + & + \\
\hline 14 & - & + & - \\
\hline 16 & + & + & + \\
\hline 17 & + & + & + \\
\hline 18 & - & - & + \\
\hline 19 & + & - & - \\
\hline
\end{tabular}

Table 4.3. Keratin members as candidate antigens responsible for the vaccination efficacy of ES-D3- derived vaccines.

The list includes lung cancer-associated keratin members identified in the serum of immunized mice or lung cancer cells (LLC). Those present in both the serum and LLC are marked in red. 


\section{DISSCUSION}

The generation of different types of tumors is a complex processes in which tumors acquire the ability of dynamic regulation and constituent cellular populations, recapitulating the complexity of organs or tissues [183]. For a long time, embryonic materials have been considered as a vaccine against cancers based on the similarities between embryos and tumors [18]. It has been found that some genes expressed in histologically poorly differentiated tumors cells are overexpressed in ESCs, and vice versa [85]. The identification of CSCs indicates that some tumor cells have the properties of stem cells to generate tumors [156]. In our study, parental ES-D3 cells display robust anchorageindependent growth capability (Figure 4.2), a key signature of tumorigenesis that correlates with tumorigenic and metastatic potential in animals [171]. This suggests that the signaling transduction pathway(s) governing cell proliferation in ESCs is similar to that in neoplastic cells. Importantly, a decrease in the pluripotency of ESCs completely abolished their anchorage-independent growth potential, lending more support for the importance of ESC pluripotency in regulating the cellular phenotypes shared with tumor cells.

Anti-tumor immunity is the most crucial factor in the prevention of tumorigenesis by the vaccination of ESCs. Furthermore, the similarity between ESC antigens and tumor antigens is considered to be responsible for inducing the immune responses against malignancy. In our study, vaccination with parental ESCs inhibited lung tumor development, whereas the differentiation of 
ESCs suppressed the efficacy of the vaccine (Figure 4.3). As the protection against lung cancer conferred by ESC vaccination likely involves a number of shared antigens, it is conceivable that differentiation could decrease expression of tumor antigens on ESCs, leading to a reduction in cross-immunity against tumors. This is in agreement with the differentiation-evoked loss of tumor cell signatures in ESCs, such as anchorage-independent growth. However, the identities of specific cross-reactive antigens and their gene expression modulation need to be investigated in future studies.

To explore the tumor-associated antigens shared between ES-D3 cells and malignant cells, we examined the comprehensive profiles of antigens specific for the antibodies induced by vaccines based on intact ES-D3 cells or exosomes of ES-D3 cells by an immunoproteomic approach (Table 4.2). This analysis reveals that ES-D3 cells and lung cancer cells share expression of a number of proteins implicated in tumorigenesis, which are candidate crossreactive antigens evoking anti-tumor immunity. Interestingly, none of the antibodies against tumor-antigens present in exosomes of ES-D3 cells (Table 4.1) are enriched in the serum from immunized mice, suggesting that those antigens are likely involved in generating anti-lung cancer immunity through other mechanisms, such as promoting cytotoxic $\mathrm{T}$ cell responses against pulmonary malignancy.

Our studies indicate the anti-tumor efficacies of both ESC cell-based or exosome-based vaccines were correlated with significantly enhanced T cell- 
mediated immune responses, including stronger Th1-mediated cytokine responses in splenic $C D 8^{+} \mathrm{T}$ cells and higher $\mathrm{CD} 8^{+} \mathrm{T} / \mathrm{T}_{\text {regs }}$ cell ratio in tumors [29], (Figures 2.10, 2.12). However, whether the humoral immune response mediated by antibodies also contributes to anti-cancer efficacy of ESC vaccination strategy remains unknown. Interestingly, a similar iPSC vaccine indeed produced antibodies specifically reactive to tumors cells [30]. Thus, it is possible that antibodies generated by ESC-based vaccine recognize the antigens on lung tumor cells to evoke ADCC-mediated anti-tumor immunity.

A large portion of antigens identified by immunoproteomic analysis are keratin family members, which are proteins forming intermediate filaments in epithelial cells [182]. Notably, the expression of keratin proteins is crucial for uncontrolled proliferation of malignant cells of epithelial origin within anaplastic cancers. Keratins have been extensively used in clinical tumor diagnosis as immunohistochemical markers since 1980 [184]. Importantly, in the assessment of different types of cancer metastases, the expression patterns of keratin subtypes could be utilized to predict the origin of primary tumors [182]. Since it was discovered that keratins are expressed in all epithelial cells [185], several members of keratins, including keratin $7,8,16,17,18$ and 19, have subsequently been identified as markers of lung cancer [186-188].

Notably, keratin 8, 16, and 17 are among the lung cancer-associated keratin family members whose levels in serum are enhanced by both the cells-based vaccine as well as the exosome-based vaccine (Table 4.3). Since these keratin 
proteins are also present in LLC cell lysate, they are likely to be the candidate antigens evoking immunity against LLC. Based on clinical research, the expression of keratin 8 is significantly higher in patients with non-small cell lung cancer (NSCLC) compared with small cell lung cancer (SCLC) patients [189]. It has been concluded that high expression of keratin 8 enhances tumor progression and indicates poor prognosis [189].

Although keratin proteins form intermediate filaments are inside cells, emerging evidence indicate that some keratin family members are localized on cell surface. For instance, the presence of keratins 8, 18 and 19 were detected on the outer surface of human mammary carcinoma cells as well as in culture medium [190]. Similarly, keratin 8 is expressed at the surface of lung carcinoma cells, but not in normal epithelial cells [191]. Furthermore, fragments of keratin 8 released by lung cancer cells were utilized as an indicator of tumor progression in clinical studies [192, 193]. Importantly, the interaction between keratin 8 and $\mathrm{MHC}$ class I was involved in $\mathrm{CD}^{+} \mathrm{T}$ cell activation in a lymph node metastatic carcinoma cell line [194]. It is conceivable that keratin 8 molecules on lung tumor cell surface are recognized by specific antibodies generated by ESC-based vaccine, resulting in recruitment of effector immune cells and subsequent tumor cell apoptosis.

As a member of the type I cytokeratins, keratin 16 has been found to associate with squamous differentiation [195]. A recent study demonstrates that keratin 16 possesses oncogenic activity to promote the tumorigenesis of lung 
adenocarcinoma and is considered to be a predictive factor of poor patients prognosis [196, 197]. Accumulating studies indicate keratin 17 is overexpressed in a number of malignancies and plays an important role in the progression of tumors [197-200]. For example, it has been found that the mRNA level of keratin 17 is significantly elevated in lung carcinoma tissues compared with normal lung tissues. Markedly, metastasis and poor survival of lung cancer patients is correlated with high expression of keratin 17 [200]. Unlike keratin 8, the presence of keratins 16 and 17 on the surface of lung tumor cells remains unknown. More studies are needed to address how keratins 16 and 17 are involved in ESC vaccine-evoked ADCC.

Overall, our studies reveal an important role of pluripotency of ESCs in antilung cancer efficacy afforded by ESC-based vaccines. Furthermore, several keratin family members were identified as candidate cross-reactive antigens to confer immunity against lung cancer. Future research is warranted to elucidate the mechanisms of immunity against lung neoplastic diseases evoked by ESCbased vaccination. 


\section{REFERENCE}

1. McGuire, S., World Cancer Report 2014. Geneva, Switzerland: World Health Organization, International Agency for Research on Cancer, WHO Press, 2015. Adv Nutr, 2016. 7(2): p. 418-9.

2. Vinay, K., A.K. Abbas, and N.J.S. Fauston, El Sevier, China, Robbins and Cotran pathologic basis of disease. 2005. 8: p. 208-221.

3. Carbone, D.P., et al., Non-Small-Cell Lung Cancer: Role of the Immune System and Potential for Immunotherapy. J Thorac Oncol, 2015. 10(7): p. 974-84.

4. Brahmer, J.R., Immune checkpoint blockade: the hope for immunotherapy as a treatment of lung cancer? Semin Oncol, 2014. 41(1): p. 126-32.

5. Silva, A.P., et al., Targeted therapies for the treatment of non-small-cell lung cancer: Monoclonal antibodies and biological inhibitors. Hum Vaccin Immunother, 2017. 13(4): p. 843-853.

6. Thomas, S. and G.C. Prendergast, Cancer Vaccines: A Brief Overview. Methods Mol Biol, 2016. 1403: p. 755-61.

7. Moticka, E.J., A historical perspective on evidence-based immunology. 2015: Newnes.

8. Lowy, D.R. and J.T. Schiller, Prophylactic human papillomavirus vaccines. J Clin Invest, 2006. 116(5): p. 1167-73.

9. Handy, C.E. and E.S. Antonarakis, Sipuleucel-T for the treatment of prostate cancer: novel insights and future directions. Future Oncology, 2018. 14(10): p. 907-917.

10. Gulley, J.L. and C.G. Drake, Immunotherapy for prostate cancer: recent advances, lessons learned, and areas for further research. Clin Cancer Res, 2011. 17(12): p. 3884-91. 
11. Murala, S., et al., Current status of immunotherapy for the treatment of lung cancer. J Thorac Dis, 2010. 2(4): p. 237-44.

12. Tagliamonte, M., et al., Antigen-specific vaccines for cancer treatment. Hum Vaccin Immunother, 2014. 10(11): p. 3332-46.

13. Al-Hajj, M. and M.F. Clarke, Self-renewal and solid tumor stem cells. Oncogene, 2004. 23(43): p. 7274-82.

14. Matsui, W., et al., Characterization of clonogenic multiple myeloma cells. Blood, 2004. 103(6): p. 2332-6.

15. Visvader, J.E. and G.J. Lindeman, Cancer stem cells in solid tumours: accumulating evidence and unresolved questions. Nat Rev Cancer, 2008. 8(10): p. 755-68.

16. Sell, S., On the stem cell origin of cancer. Am J Pathol, 2010. 176(6): p. 2584-494.

17. Kim, W.-T. and C.J. Ryu, Cancer stem cell surface markers on normal stem cells. BMB Reports, 2017. 50(6): p. 285-298.

18. Brewer, B.G., et al., Embryonic vaccines against cancer: an early history. Exp Mol Pathol, 2009. 86(3): p. 192-7.

19. Stonehill, E.H. and A. Bendich, Retrogenetic expression: the reappearance of embryonal antigens in cancer cells. Nature, 1970. 228(5269): p. 370-2.

20. Hirszfeld, L., W. Halberówna, and J.J. Laskowski, Untersuchungen über die serologischen Eigenschaften der Gewebe: über serologische Eigenschaften der Neubildungen. 1929.

21. Laurence, D. and A.M.J.B.j.o.c. Neville, Foetal antigens and their role in the diagnosis and clinical management of human neoplasms: a review. 1972. 26(5): p. 335.

22. Adinolfi, M. and M.H. Lessof, Cancer, oncogenes and oncofetal antigens. Q J Med, 1985. 54(215): p. 193-204.

23. Bendich, A., E. Borenfreund, and E.H. Stonehill, Protection of adult mice against tumor challenge by immunization with irradiated adult skin or embryo cells. J Immunol, 1973. 111(1): p. 284-5. 
24. Klavins, J.V., R. Mesa-Tejada, and M. Weiss, Human carcinoma antigens cross reacting with anti-embryonic antibodies. Nat New Biol, 1971. 234(48): p. 153-4.

25. Li, Y., et al., Vaccination with human pluripotent stem cells generates a broad spectrum of immunological and clinical responses against colon cancer. Stem Cells, 2009. 27(12): p. 3103-11.

26. Yaddanapudi, K., R.A. Mitchell, and J.W. Eaton, Cancer vaccines: Looking to the future. Oncoimmunology, 2013. 2(3): p. e23403.

27. Dranoff, G., et al., Vaccination with irradiated tumor cells engineered to secrete murine granulocyte-macrophage colony-stimulating factor stimulates potent, specific, and long-lasting anti-tumor immunity. Proc Natl Acad Sci U S A, 1993. 90(8): p. 3539-43.

28. Codony-Servat, J. and R. Rosell, Cancer stem cells and immunoresistance: clinical implications and solutions. Transl Lung Cancer Res, 2015. 4(6): p. 689-703.

29. Yaddanapudi, K., et al., Vaccination with embryonic stem cells protects against lung cancer: is a broad-spectrum prophylactic vaccine against cancer possible? PLoS One, 2012. 7(7): p. e42289.

30. Kooreman, N.G., et al., Autologous iPSC-Based Vaccines Elicit Antitumor Responses In Vivo. Cell Stem Cell, 2018. 22(4): p. 501-513 e7.

31. Dong, W., et al., Administration of embryonic stem cells generates effective antitumor immunity in mice with minor and heavy tumor load. Cancer immunology, immunotherapy, 2010. 59(11): p. 1697-1705.

32. Schneble, E., et al., Peptide-Based cancer vaccine strategies and clinical results, in Vaccine Design. 2016, Springer. p. 797-817.

33. Mittal, D., et al., New insights into cancer immunoediting and its three component phases-elimination, equilibrium and escape. Current opinion in immunology, 2014. 27: p. 16-25.

34. Temizoz, B., E. Kuroda, and K.J. Ishii, Vaccine adjuvants as potential cancer immunotherapeutics. International immunology, 2016. 28(7): p. 329-338.

35. Bowen, W.S., et al., Current challenges for cancer vaccine adjuvant development. Expert review of vaccines, 2018. 17(3): p. 207-215. 
36. Bradley, T.R. and D. Metcalf, The growth of mouse bone marrow cells in vitro. Aust J Exp Biol Med Sci, 1966. 44(3): p. 287-99.

37. Francisco-Cruz, A., et al., Granulocyte-macrophage colony-stimulating factor: not just another haematopoietic growth factor. Med Oncol, 2014. 31(1): p. 774.

38. Fanger, N.A., et al., Activation of human $T$ cells by major histocompatability complex class II expressing neutrophils: proliferation in the presence of superantigen, but not tetanus toxoid. 1997. 89(11): p. 4128-4135.

39. O'Mahony, D.S., et al., Differential constitutive and cytokine-modulated expression of human Toll-like receptors in primary neutrophils, monocytes, and macrophages. Int J Med Sci, 2008. 5(1): p. 1-8.

40. Miah, M.A., et al., CISH is induced during DC development and regulates DC-mediated CTL activation. 2012. 42(1): p. 58-68.

41. Hornell, T.M., et al., Regulation of the class II MHC pathway in primary human monocytes by granulocyte-macrophage colony-stimulating factor. 2003. 171(5): p. 2374-2383.

42. Higano, C.S., et al., Integrated data from 2 randomized, double-blind, placebo-controlled, phase 3 trials of active cellular immunotherapy with sipuleucel-T in advanced prostate cancer. 2009. 115(16): p. 3670-3679.

43. Olivares, J., et al., Phase I trial of TGF- $\beta 2$ antisense GM-CSF genemodified autologous tumor cell (TAG) vaccine. 2011. 17(1): p. 183-192.

44. Sun, X., et al., Co-expression of granulocyte-macrophage colonystimulating factor with antigen enhances humoral and tumor immunity after DNA vaccination. 2002. 20(9-10): p. 1466-1474.

45. Holt, G.E. and M.L.J.C.I.c. Disis, Immune Modulation as a Therapeutic Strategy for Non-Small-Cell Lung Cancer. 2008. 9: p. S13-S19.

46. Nemunaitis, J., et al., Granulocyte-macrophage colony-stimulating factor gene-modified autologous tumor vaccines in non-small-cell lung cancer. 2004. 96(4): p. 326-331.

47. Aliper, A.M., et al., A role for G-CSF and GM-CSF in nonmyeloid cancers. Cancer Med, 2014. 3(4): p. 737-46. 
48. Khanna, S., et al., Tumor-Derived GM-CSF Promotes Granulocyte Immunosuppression in Mesothelioma Patients. Clin Cancer Res, 2018. 24(12): p. 2859-2872.

49. Oppmann, B., et al., Novel p19 protein engages IL-12p40 to form a cytokine, IL-23, with biological activities similar as well as distinct from IL-12. Immunity, 2000. 13(5): p. 715-25.

50. Coulie, P.G. and P. van der Bruggen, T-cell responses of vaccinated cancer patients. Curr Opin Immunol, 2003. 15(2): p. 131-7.

51. Overwijk, W.W., et al., Immunological and antitumor effects of IL-23 as a cancer vaccine adjuvant. J Immunol, 2006. 176(9): p. 5213-22.

52. Shirota, H., D. Tross, and D.M. Klinman, CpG oligonucleotides as cancer vaccine adjuvants. Vaccines, 2015. 3(2): p. 390-407.

53. Powell, B.S., A.K. Andrianov, and P.C. Fusco, Polyionic vaccine adjuvants: another look at aluminum salts and polyelectrolytes. Clin Exp Vaccine Res, 2015. 4(1): p. 23-45.

54. Temizoz, B., E. Kuroda, and K.J. Ishii, Vaccine adjuvants as potential cancer immunotherapeutics. Int Immunol, 2016. 28(7): p. 329-38.

55. Yáñez-Mó, M., et al., Biological properties of extracellular vesicles and their physiological functions. 2015. 4(1): p. 27066.

56. Chargaff, E. and R.J.J.B.C. West, The biological significance of the thromboplastic protein of blood. 1946. 166(1): p. 189-197.

57. Stegmayr, B. and G.J.U.r. Ronquist, Promotive effect on human sperm progressive motility by prostasomes. 1982. 10(5): p. 253-257.

58. Harding, C., J. Heuser, and P.J.E.j.o.c.b. Stahl, Endocytosis and intracellular processing of transferrin and colloidal gold-transferrin in rat reticulocytes: demonstration of a pathway for receptor shedding. 1984. 35(2): p. 256-263.

59. Valadi, H., et al., Exosome-mediated transfer of mRNAs and microRNAs is a novel mechanism of genetic exchange between cells. 2007. 9(6): p. 654. 
60. Van der Pol, E., et al., Recent developments in the nomenclature, presence, isolation, detection and clinical impact of extracellular vesicles. 2016. 14(1): p. 48-56.

61. Couzin, J., Cell biology: The ins and outs of exosomes. Science, 2005. 308(5730): p. 1862-3.

62. Thakur, B.K., et al., Double-stranded DNA in exosomes: a novel biomarker in cancer detection. Cell Res, 2014. 24(6): p. 766-9.

63. Wang, M., et al., Role of tumor microenvironment in tumorigenesis. Journal of Cancer, 2017. 8(5): p. 761.

64. Li, X.B., et al., Role of exosomes in immune regulation. 2006. 10(2): p. 364-375.

65. Mears, R., et al., Proteomic analysis of melanoma-derived exosomes by two-dimensional polyacrylamide gel electrophoresis and mass spectrometry. Proteomics, 2004. 4(12): p. 4019-31.

66. Wolfers, J., et al., Tumor-derived exosomes are a source of shared tumor rejection antigens for CTL cross-priming. Nature medicine, 2001. 7(3): p. 297-303.

67. lero, M., et al., Tumour-released exosomes and their implications in cancer immunity. Cell Death \& Differentiation, 2008. 15(1): p. 80-88.

68. Yang, H., L. Sun, and Y. Mao, The role of exosomes in tumor immunity. Annals of translational medicine, 2018. 6(Suppl 2).

69. Munich, S., et al., Dendritic cell exosomes directly kill tumor cells and activate natural killer cells via TNF superfamily ligands. Oncoimmunology, 2012. 1(7): p. 1074-1083.

70. Zhu, L., et al., Exosomes derived from natural killer cells exert therapeutic effect in melanoma. Theranostics, 2017. 7(10): p. 2732.

71. Raposo, G., et al., B lymphocytes secrete antigen-presenting vesicles. J Exp Med, 1996. 183(3): p. 1161-72.

72. Pitt, J.M., et al., Dendritic cell-derived exosomes for cancer therapy. The Journal of clinical investigation, 2016. 126(4): p. 1224-1232. 
73. Escudier, B., et al., Vaccination of metastatic melanoma patients with autologous dendritic cell (DC) derived-exosomes: results of thefirst phase I clinical trial. Journal of translational medicine, 2005. 3(1): p. 10.

74. Morse, M.A., et al., A phase I study of dexosome immunotherapy in patients with advanced non-small cell lung cancer. Journal of translational medicine, 2005. 3(1): p. 9.

75. Besse, B., et al., Dendritic cell-derived exosomes as maintenance immunotherapy after first line chemotherapy in NSCLC. Oncoimmunology, 2016. 5(4): p. e1071008.

76. Todryk, S., et al., Heat shock protein 70 induced during tumor cell killing induces Th1 cytokines and targets immature dendritic cell precursors to enhance antigen uptake. J Immunol, 1999. 163(3): p. 1398-408.

77. Udono, H. and P.K. Srivastava, Comparison of tumor-specific immunogenicities of stress-induced proteins gp96, hsp90, and hsp70. J Immunol, 1994. 152(11): p. 5398-403.

78. lero, M., et al., Tumour-released exosomes and their implications in cancer immunity. Cell Death Differ, 2008. 15(1): p. 80-8.

79. Dai, S., et al., Phase I clinical trial of autologous ascites-derived exosomes combined with GM-CSF for colorectal cancer. Molecular therapy, 2008. 16(4): p. 782-790.

80. Ichim, T.E., et al., Exosomes as a tumor immune escape mechanism: possible therapeutic implications. J Transl Med, 2008. 6: p. 37.

81. Valenti, R., et al., Tumor-released microvesicles as vehicles of immunosuppression. Cancer Res, 2007. 67(7): p. 2912-5.

82. Dalgleish, A.G.J.E.r.o.v., Cancer vaccines as a therapeutic strategy. 2004. 3(6): p. 665-668.

83. Kooreman, N.G., et al., Autologous iPSC-based vaccines elicit antitumor responses in vivo. 2018. 22(4): p. 501-513. e7.

84. Triolo, V.A., Nineteenth Century Foundations Of Cancer Research Advances In Tumor Pathology, Nomenclature, And Theories Of Oncogenesis. Cancer Res, 1965. 25: p. 75-106. 
85. Ben-Porath, I., et al., An embryonic stem cell-like gene expression signature in poorly differentiated aggressive human tumors. Nat Genet, 2008. 40(5): p. 499-507.

86. Colombo, M., G. Raposo, and C. Thery, Biogenesis, secretion, and intercellular interactions of exosomes and other extracellular vesicles. Annu Rev Cell Dev Biol, 2014. 30: p. 255-89.

87. Gehrmann, U., et al., Harnessing the exosome-induced immune response for cancer immunotherapy. Semin Cancer Biol, 2014. 28: p. 58-67.

88. Thery, C., L. Zitvogel, and S. Amigorena, Exosomes: composition, biogenesis and function. Nat Rev Immunol, 2002. 2(8): p. 569-79.

89. Valadi, H., et al., Exosome-mediated transfer of mRNAs and microRNAs is a novel mechanism of genetic exchange between cells. Nat Cell Biol, 2007. 9(6): p. 654-9.

90. Yanez-Mo, M., et al., Biological properties of extracellular vesicles and their physiological functions. J Extracell Vesicles, 2015. 4: p. 27066.

91. Lener, T., et al., Applying extracellular vesicles based therapeutics in clinical trials - an ISEV position paper. J Extracell Vesicles, 2015. 4: p. 30087.

92. Witwer, K.W., et al., Standardization of sample collection, isolation and analysis methods in extracellular vesicle research. J Extracell Vesicles, 2013. 2.

93. Armstrong, J.P., M.N. Holme, and M.M. Stevens, Re-Engineering Extracellular Vesicles as Smart Nanoscale Therapeutics. ACS Nano, 2017. 11(1): p. 69-83.

94. Tremml, G., M. Singer, and R. Malavarca, Culture of mouse embryonic stem cells. Curr Protoc Stem Cell Biol, 2008. Chapter 1: p. Unit 1C 4.

95. Kirsch, P., et al., Time course of fluorescence intensity and protein expression in HeLa cells stably transfected with hrGFP. Mol Cells, 2003. 15(3): p. 341-8.

96. Zeng, X., et al., Stable expression of hrGFP by mouse embryonic stem cells: promoter activity in the undifferentiated state and during dopaminergic neural differentiation. Stem Cells, 2003. 21(6): p. 647-53. 
97. Baldwin, R.W., D. Glaves, and B.M. Vose, Embryonic antigen expression in chemically induced rat hepatomas and sarcomas. Int J Cancer, 1972. 10(2): p. 233-43.

98. Baldwin, R.W., et al., Tumour specific and embryonic antigen expression of chemically induced rat tumours. Ann Inst Pasteur (Paris), 1972. 122(4): p. $715-28$.

99. Mali, P., et al., Improved efficiency and pace of generating induced pluripotent stem cells from human adult and fetal fibroblasts. Stem Cells, 2008. 26(8): p. 1998-2005.

100. Chung, S., et al., Genetic engineering of mouse embryonic stem cells by Nurr1 enhances differentiation and maturation into dopaminergic neurons. Eur J Neurosci, 2002. 16(10): p. 1829-38.

101. Caracciolo, D., S.C. Clark, and G. Rovera, Human interleukin-6 supports granulocytic differentiation of hematopoietic progenitor cells and acts synergistically with GM-CSF. Blood, 1989. 73(3): p. 666-70.

102. Zhang, X., et al., Exosomes for Immunoregulation and Therapeutic Intervention in Cancer. J Cancer, 2016. 7(9): p. 1081-7.

103. Bosch, S., et al., Trehalose prevents aggregation of exosomes and cryodamage. Sci Rep, 2016. 6: p. 36162.

104. Thery, C., et al., Isolation and characterization of exosomes from cell culture supernatants and biological fluids. Curr Protoc Cell Biol, 2006. Chapter 3: p. Unit 322.

105. hebdomadaire, W.H.O.J.W.E.R.R.é., Human papillomavirus vaccines: WHO position paper. 2009. 84(15): p. 118-131.

106. Dranoff, G., GM-CSF-based cancer vaccines. Immunol Rev, 2002. 188: p. 147-54.

107. Bencherif, S.A., et al., Injectable cryogel-based whole-cell cancer vaccines. Nat Commun, 2015. 6: p. 7556.

108. Jung, M.K. and J.Y. Mun, Sample preparation and imaging of exosomes by transmission electron microscopy. JoVE (Journal of Visualized Experiments), 2018(131): p. e56482. 
109. Dragovic, R.A., et al., Sizing and phenotyping of cellular vesicles using Nanoparticle Tracking Analysis. Nanomedicine: Nanotechnology, Biology and Medicine, 2011. 7(6): p. 780-788.

110. Kunigelis, K.E. and M.W. Graner, The Dichotomy of Tumor Exosomes (TEX) in Cancer Immunity: Is It All in the ConTEXt? Vaccines (Basel), 2015. 3(4): p. 1019-51.

111. Borrello, I. and D. Pardoll, GM-CSF-based cellular vaccines: a review of the clinical experience. Cytokine Growth Factor Rev, 2002. 13(2): p. 18593.

112. Soiffer, R., et al., Vaccination with irradiated, autologous melanoma cells engineered to secrete granulocyte-macrophage colony-stimulating factor by adenoviral-mediated gene transfer augments antitumor immunity in patients with metastatic melanoma. J Clin Oncol, 2003. 21(17): p. 3343-50.

113. Viaud, S., et al., Dendritic cell-derived exosomes promote natural killer cell activation and proliferation: a role for NKG2D ligands and IL15Ralpha. PLoS One, 2009. 4(3): p. e4942.

114. Zitvogel, L., et al., Eradication of established murine tumors using a novel cell-free vaccine: dendritic cell-derived exosomes. Nat Med, 1998. 4(5): p. 594-600.

115. Pichler, W.J., Adverse side-effects to biological agents. Allergy, 2006. 61(8): p. 912-20.

116. Aubin, F., F. Carbonnel, and D. Wendling, The complexity of adverse side-effects to biological agents. J Crohns Colitis, 2013. 7(4): p. 257-62.

117. Hedlund, M., et al., Human placenta expresses and secretes NKG2D ligands via exosomes that down-modulate the cognate receptor expression: evidence for immunosuppressive function. J Immunol, 2009. 183(1): p. 340-51.

118. Nugent, W.C., et al., Non-small cell lung cancer at the extremes of age: impact on diagnosis and treatment. The Annals of thoracic surgery, 1997. 63(1): p. 193-197.

119. Radzikowska, E., K. Roszkowski, and P. Głaz, Lung cancer in patients under 50 years old. Lung cancer, 2001. 33(2-3): p. 203-211. 
120. Domagala-Kulawik, J., I. Osinska, and G. Hoser, Mechanisms of immune response regulation in lung cancer. Transl Lung Cancer Res, 2014. 3(1): p. 15-22.

121. Zou, W., Immunosuppressive networks in the tumour environment and their therapeutic relevance. Nat Rev Cancer, 2005. 5(4): p. 263-74.

122. Rizvi, N.A., et al., Activity and safety of nivolumab, an anti-PD-1 immune checkpoint inhibitor, for patients with advanced, refractory squamous non-small-cell lung cancer (CheckMate 063): a phase 2, single-arm trial. Lancet Oncol, 2015. 16(3): p. 257-65.

123. Thungappa, S., et al., Immune checkpoint inhibitors in lung cancer: the holy grail has not yet been found. ESMO Open, 2017. 2(1): p. e000162.

124. Mellman, I., G. Coukos, and G. Dranoff, Cancer immunotherapy comes of age. Nature, 2011. 480(7378): p. 480-9.

125. Steeg, P.S., Tumor metastasis: mechanistic insights and clinical challenges. Nat Med, 2006. 12(8): p. 895-904.

126. Khan, N. and $\mathrm{H}$. Mukhtar, Cancer and metastasis: prevention and treatment by green tea. Cancer Metastasis Rev, 2010. 29(3): p. 435-45.

127. Stella, G.M., et al., Lung-Seeking Metastases. Cancers (Basel), 2019. 11(7).

128. Fischer, A.H., et al., Hematoxylin and eosin staining of tissue and cell sections. CSH Protoc, 2008. 2008: p. pdb prot4986.

129. Pinto, A., S. Morello, and R. Sorrentino, Lung cancer and Toll-like receptors. Cancer Immunol Immunother, 2011. 60(9): p. 1211-20.

130. Igney, F.H. and P.H. Krammer, Immune escape of tumors: apoptosis resistance and tumor counterattack. J Leukoc Biol, 2002. 71(6): p. 90720.

131. Elkin, M. and I. Vlodavsky, Tail vein assay of cancer metastasis. Curr Protoc Cell Biol, 2001. Chapter 19: p. Unit 192.

132. Meuwissen, R. and A. Berns, Mouse models for human lung cancer. Genes Dev, 2005. 19(6): p. 643-64. 
133. Man, Y.G., et al., Tumor-infiltrating immune cells promoting tumor invasion and metastasis: existing theories. J Cancer, 2013. 4(1): p. 8495.

134. Farhood, B., M. Najafi, and K. Mortezaee, CD8(+) cytotoxic T lymphocytes in cancer immunotherapy: A review. J Cell Physiol, 2019. 234(6): p. 8509-8521.

135. Oleinika, K., et al., Suppression, subversion and escape: the role of regulatory T cells in cancer progression. Clin Exp Immunol, 2013. 171(1): p. $36-45$.

136. Condamine, T., et al., Regulation of tumor metastasis by myeloid-derived suppressor cells. Annu Rev Med, 2015. 66: p. 97-110.

137. Qian, B.Z. and J.W. Pollard, Macrophage diversity enhances tumor progression and metastasis. Cell, 2010. 141(1): p. 39-51.

138. Wang, C., et al., Characterization of murine macrophages from bone marrow, spleen and peritoneum. BMC Immunol, 2013. 14: p. 6.

139. Schmall, A., et al., Macrophage and cancer cell cross-talk via CCR2 and CX3CR1 is a fundamental mechanism driving lung cancer. Am J Respir Crit Care Med, 2015. 191(4): p. 437-47.

140. Wang, S.S., et al., Tumor-infiltrating B cells: their role and application in anti-tumor immunity in lung cancer. Cell Mol Immunol, 2019. 16(1): p. 618.

141. Ito, N., et al., Prognostic significance of T helper 1 and 2 and T cytotoxic 1 and 2 cells in patients with non-small cell lung cancer. Anticancer Res, 2005. 25(3B): p. 2027-31.

142. Asadzadeh, Z., et al., The paradox of Th17 cell functions in tumor immunity. Cell Immunol, 2017. 322: p. 15-25.

143. Maimela, N.R., S. Liu, and Y. Zhang, Fates of CD8+ T cells in Tumor Microenvironment. Comput Struct Biotechnol J, 2019. 17: p. 1-13.

144. Chambers, A.F., A.C. Groom, and I.C. MacDonald, Dissemination and growth of cancer cells in metastatic sites. Nat Rev Cancer, 2002. 2(8): p. $563-72$. 
145. Elkin, M. and I. Vlodavsky, Tail vein assay of cancer metastasis. Curr Protoc Cell Biol, 2001. Chapter 19: p. 192 1-19 27.

146. Gomez-Cuadrado, L., et al., Mouse models of metastasis: progress and prospects. Dis Model Mech, 2017. 10(9): p. 1061-1074.

147. Al-Hajj, M., et al., Therapeutic implications of cancer stem cells. Curr Opin Genet Dev, 2004. 14(1): p. 43-7.

148. Curiel, T.J., Regulatory $\mathrm{T}$ cells and treatment of cancer. Curr Opin Immunol, 2008. 20(2): p. 241-6.

149. Ouzounova, M., et al., Monocytic and granulocytic myeloid derived suppressor cells differentially regulate spatiotemporal tumour plasticity during metastatic cascade. Nat Commun, 2017. 8: p. 14979.

150. Stix, G., A malignant flame. Understanding chronic inflammation, which contributes to heart disease, Alzheimer's and a variety of other ailments, may be a key to unlocking the mysteries of cancer. Sci Am, 2007. 297(1): p. 60-7.

151. Takanami, I., K. Takeuchi, and S. Kodaira, Tumor-associated macrophage infiltration in pulmonary adenocarcinoma: association with angiogenesis and poor prognosis. Oncology, 1999. 57(2): p. 138-42.

152. Lin, Y., J. $\mathrm{Xu}$, and $\mathrm{H}$. Lan, Tumor-associated macrophages in tumor metastasis: biological roles and clinical therapeutic applications. J Hematol Oncol, 2019. 12(1): p. 76.

153. Zhu, J. and W.E. Paul, CD4 T cells: fates, functions, and faults. Blood, 2008. 112(5): p. 1557-69.

154. Singh, B., et al., Modulation of autoimmune diseases by interleukin (IL)17 producing regulatory T helper (Th17) cells. Indian J Med Res, 2013. 138(5): p. $591-4$

155. Hodge, G., et al., Lung cancer is associated with decreased expression of perforin, granzyme B and interferon (IFN)-gamma by infiltrating lung tissue T cells, natural killer (NK) T-like and NK cells. Clin Exp Immunol, 2014. 178(1): p. 79-85.

156. Reya, T., et al., Stem cells, cancer, and cancer stem cells. Nature, 2001. 414(6859): p. 105-11. 
157. Steplewski, Z., et al., Biological activity of human-mouse $\lg G 1, \lg G 2$, $\lg \mathrm{G} 3$, and IgG4 chimeric monoclonal antibodies with antitumor specificity. Proc Natl Acad Sci U S A, 1988. 85(13): p. 4852-6.

158. Velders, M.P., et al., The impact of antigen density and antibody affinity on antibody-dependent cellular cytotoxicity: relevance for immunotherapy of carcinomas. Br J Cancer, 1998. 78(4): p. 478-83.

159. Flieger, D., et al., Enhancement of antibody dependent cellular cytotoxicity (ADCC) by combination of cytokines. Hybridoma, 1999. 18(1): p. 63-8.

160. Wang, W., et al., NK Cell-Mediated Antibody-Dependent Cellular Cytotoxicity in Cancer Immunotherapy. Front Immunol, 2015. 6: p. 368.

161. Weiskopf, K. and I.L. Weissman, Macrophages are critical effectors of antibody therapies for cancer. MAbs, 2015. 7(2): p. 303-10.

162. Matlung, H.L., et al., Neutrophils Kill Antibody-Opsonized Cancer Cells by Trogoptosis. Cell Rep, 2018. 23(13): p. 3946-3959 e6.

163. Kohrt, H.E., et al., Combination strategies to enhance antitumor ADCC. Immunotherapy, 2012. 4(5): p. 511-27.

164. Mohamad, O., et al., Efficient neuronal differentiation of mouse ES and iPS cells using a rotary cell culture protocol. Differentiation, 2013. 86(45): p. 149-58.

165. Slawek, S., et al., Pluripotency transcription factors in lung cancer-a review. Tumour Biol, 2016. 37(4): p. 4241-9.

166. Soprano, D.R., B.W. Teets, and K.J. Soprano, Role of retinoic acid in the differentiation of embryonal carcinoma and embryonic stem cells. Vitam Horm, 2007. 75: p. 69-95.

167. Czechanski, A., et al., Derivation and characterization of mouse embryonic stem cells from permissive and nonpermissive strains. Nat Protoc, 2014. 9(3): p. 559-74.

168. Chklovskii, D.B., Synaptic connectivity and neuronal morphology: two sides of the same coin. Neuron, 2004. 43(5): p. 609-17. 
169. Berrill, A., et al., Assessment of stem cell markers during long-term culture of mouse embryonic stem cells. Cytotechnology, 2004. 44(1-2): p. 77-91.

170. Ohtsuka, S. and S. Dalton, Molecular and biological properties of pluripotent embryonic stem cells. Gene Ther, 2008. 15(2): p. 74-81.

171. Mori, S., et al., Anchorage-independent cell growth signature identifies tumors with metastatic potential. Oncogene, 2009. 28(31): p. 2796-805.

172. Borowicz, S., et al., The soft agar colony formation assay. J Vis Exp, 2014(92): p. e51998.

173. Taddei, M.L., et al., Anoikis: an emerging hallmark in health and diseases. J Pathol, 2012. 226(2): p. 380-93.

174. Kumari, S., et al., CD151-A Striking Marker for Cancer Therapy. Biomark Cancer, 2015. 7: p. 7-11.

175. Gutschner, T., et al., Insulin-like growth factor 2 mRNA-binding protein 1 (IGF2BP1) is an important protumorigenic factor in hepatocellular carcinoma. Hepatology, 2014. 59(5): p. 1900-11.

176. Patriarca, C., et al., Epithelial cell adhesion molecule expression (CD326) in cancer: a short review. Cancer Treat Rev, 2012. 38(1): p. 68-75.

177. Lai, Q., et al., Alpha-fetoprotein and novel tumor biomarkers as predictors of hepatocellular carcinoma recurrence after surgery: a brilliant star raises again. Int J Hepatol, 2012. 2012: p. 893103.

178. Glas, A.S., et al., Tumor markers in the diagnosis of primary bladder cancer. A systematic review. J Urol, 2003. 169(6): p. 1975-82.

179. Kornberg, A. and A. Polliack, Serum lactic dehydrogenase (LDH) levels in acute leukemia: marked elevations in lymphoblastic leukemia. Blood, 1980. 56(3): p. 351-5.

180. Tandon, M., S.V. Vemula, and S.K. Mittal, Emerging strategies for EphA2 receptor targeting for cancer therapeutics. Expert Opin Ther Targets, 2011. 15(1): p. 31-51.

181. Gates, K.V., A.J. Dalgliesh, and L.G. Griffiths, Antigenicity of Bovine Pericardium Determined by a Novel Immunoproteomic Approach. Sci Rep, 2017. 7(1): p. 2446. 
182. Karantza, V., Keratins in health and cancer: more than mere epithelial cell markers. Oncogene, 2011. 30(2): p. 127-38.

183. Hanahan, D. and R.A. Weinberg, Hallmarks of cancer: the next generation. Cell, 2011. 144(5): p. 646-74.

184. Moll, R., M. Divo, and L. Langbein, The human keratins: biology and pathology. Histochem Cell Biol, 2008. 129(6): p. 705-33.

185. Bragulla, H.H. and D.G. Homberger, Structure and functions of keratin proteins in simple, stratified, keratinized and cornified epithelia. J Anat, 2009. 214(4): p. 516-59.

186. Wetzels, R.H., et al., Laminin and type VII collagen distribution in different types of human lung carcinoma: correlation with expression of keratins 14, 16, 17 and 18. Histopathology, 1992. 20(4): p. 295-303.

187. MOLENGRAFT, F.V.D., et al., OV-TL $12 / 30$ (keratin 7 antibody) is a marker of glandular differentiation in lung cancer. 1993. 22(1): p. 35-38.

188. Gao, J., et al., Serum cytokeratin 19 fragment, CK19-2G2, as a newly identified biomarker for lung cancer. 2014. 9(7): p. e101979.

189. Fukunaga, Y., et al., Expression of cytokeratin 8 in lung cancer cell lines and measurement of serum cytokeratin 8 in lung cancer patients. Lung Cancer, 2002. 38(1): p. 31-8.

190. Godfroid, E., et al., Cytokeratins are exposed on the outer surface of established human mammary carcinoma cells. J Cell Sci, 1991. 99 ( Pt 3): p. 595-607.

191. Blobel, G.A., et al., Cytokeratins in normal lung and lung carcinomas. Virchows Archiv B, 1984. 45(1): p. 407-429.

192. Barak, V., et al., Clinical utility of cytokeratins as tumor markers. Clinical biochemistry, 2004. 37(7): p. 529-540.

193. Linder, S., Cytokeratin markers come of age. Tumor Biology, 2007. 28(4): p. 189-195.

194. Wu, M.S., et al., Cytokeratin 8-MHC class I interactions: a potential novel immune escape phenotype by a lymph node metastatic carcinoma cell line. Biochem Biophys Res Commun, 2013. 441(3): p. 618-23. 
195. Smedts, F., et al., Keratin expression in cervical cancer. Am J Pathol, 1992. 141(2): p. 497-511.

196. Yuanhua, L., et al., TFAP2A Induced KRT16 as an Oncogene in Lung Adenocarcinoma via EMT. Int J Biol Sci, 2019. 15(7): p. 1419-1428.

197. Depianto, D., et al., Keratin 17 promotes epithelial proliferation and tumor growth by polarizing the immune response in skin. Nat Genet, 2010. 42(10): p. 910-4.

198. Kim, C.Y., et al., Proteomic analysis reveals overexpression of moesin and cytokeratin 17 proteins in colorectal carcinoma. Oncol Rep, 2012. 27(3): p. 608-20.

199. Kolokythas, A., et al., Analysis of RNA from brush cytology detects changes in B2M, CYP1B1 and KRT17 levels with OSCC in tobacco users. Oral Oncol, 2011. 47(6): p. 532-6.

200. Liu, J., et al., Keratin 17 Promotes Lung Adenocarcinoma Progression by Enhancing Cell Proliferation and Invasion. Med Sci Monit, 2018. 24: p. 4782-4790. 


\section{LIST OF ABBREVIATIONS}

$\begin{array}{ll}\text { ADCC } & \text { antibody-dependent cellular cytotoxicity } \\ \text { AFP } & \text { alpha fetoprotein } \\ \text { ALP } & \text { alkaline phosphatase } \\ \text { ALT } & \text { alanine transaminase } \\ \text { APCs } & \text { antigen-presenting cells } \\ \text { BUN } & \text { blood urea nitrogen } \\ \text { CAR } & \text { chimeric antigen receptor } \\ \text { CBC } & \text { complete blood count } \\ \text { CEA } & \text { carcinoembryonic antigen } \\ \text { CICs } & \text { cancer-initiating cells } \\ \text { CISH } & \text { cytokine inducible SH2domain } \\ \text { CRC } & \text { colorectal carcinoma } \\ \text { CSCs } & \text { cancer stem cells } \\ \text { CSF } & \text { colony-stimulating factors } \\ \text { CTA } & \text { cancer/testis antigen } \\ \text { CTL } & \text { cytotoxic T lymphocyte } \\ \text { CTLA } & \text { cytotoxic T lymphocyte antigen }\end{array}$




\begin{tabular}{|c|c|}
\hline DCs & dendritic cells \\
\hline Dexosomes & dendritic cell-derived exosomes \\
\hline EF1a & elongation factor- $1 \mathrm{a}$ \\
\hline EGFR & epidermal growth factor receptor \\
\hline ESCs & embryonic stem cells \\
\hline ES-exo/GM-CSF & ESC-derived exosomes bearing GM-CSF \\
\hline EVs & extracellular vesicles \\
\hline FDA & Food and Drug Administration \\
\hline GM-CSF & granulocyte macrophage-colony stimulating factor \\
\hline G-MDSCs & granulocytic myeloid derived suppressor cells \\
\hline HBV & human hepatitis $B$ virus \\
\hline HPV & human papilloma virus \\
\hline HSPs & heat shock proteins \\
\hline IACUC & institutional animal care and use committee \\
\hline ICAM-1 & intercellular cell-adhesion molecule-1 \\
\hline IL & interleukin \\
\hline INF & interferon \\
\hline iPSCs & induced pluripotent stem cells \\
\hline LDH & lactate dehydrogenase \\
\hline LIF & leukemia inhibitory factor \\
\hline LLC & Lewis lung carcinoma \\
\hline MAGE & melanoma-associated antigen \\
\hline
\end{tabular}




\begin{tabular}{|c|c|}
\hline MDSCs & myeloid derived suppressor cells \\
\hline $\mathrm{MHC}$ & major histocompatibility complex \\
\hline M-MDSCs & monocytic myeloid derived suppressor cells \\
\hline MVBs & multivesicular bodies \\
\hline NK & natural killer \\
\hline NOD-SCID & non-obese diabetic severe \\
\hline & immunodeficiency \\
\hline NSAID & non-steroidal anti-inflammatory drug \\
\hline NSCLC & non-small cell lung carcinoma \\
\hline PD-1 & programmed cell death protein 1 \\
\hline PDI & protein disulfide isomerase \\
\hline PD-L1 & programmed cell death protein ligand 1 \\
\hline PSA & prostate-specific antigen \\
\hline s.c. & subcutaneous \\
\hline STAT5 & signal transducer and activator of transcription 5 \\
\hline STR & short tandem repeat \\
\hline TAAs & tumor-associated antigens \\
\hline$T_{c}$ & cytotoxic T cells \\
\hline TEM & transmission electron microscopy \\
\hline Texosomes & tumor-derived exosomes \\
\hline TGF & transforming growth factor \\
\hline Th & T helper cell \\
\hline
\end{tabular}


TIL

TLR2

TLR4

TNF- $\alpha$

TRAIL

Tregs tumor-infiltrating lymphocytes

toll like receptor 2

toll like receptor 4

tumor necrosis factor alpha

TNF-related apoptosis-inducing ligand

T regulatory cells 
CURRICULUM VITAE

\author{
Shuhan Meng \\ 505 South Hancock Street, CTRB 433A \\ Louisville, KY, 40202 \\ $502-956-5730$ \\ s0meng02@louisville.edu
}

\title{
Education
}

08/2016-05/2020 Ph.D. in Pharmacology and Toxicology

University of Louisville, Louisville, Kentucky, USA

09/2014-06/2016 M.D. in Internal Medicine

Jilin University, Changchun, China

09/2009-06/2014 B.S. in Clinical Medicine

Jilin University, Changchun, China

\section{Positions}

05/2017-05/2020 Ph.D. Candidate, Department of Pharmacology and Toxicology, University of Louisville, Louisville, Kentucky, USA 
08/2016-05/2017 Predoctoral student, Department of Pharmacology and Toxicology, University of Louisville, Louisville, Kentucky, USA

09/2014-06/2016 Internship as resident doctor, Second Hospital of Jilin University, Changchun, China

\section{Certification and License}

Medical License and Board Certificate in China

\section{Professional Membership}

02/2019- Member, American Society for Pharmacology and Experimental Therapeutics (ASPET)

11/2019- Member, American Association for the Advancement of Science (AAAS)

\section{Honors and Awards}

04/2019 Travel award, Graduate School Council University of Louisville

04/2019 Travel award, IDeA National Resource for Proteomics Workshop, National Institutes of Health

04/2019 The first place award for poster presentation, Experimental Biology Meeting

06/2016 Outstanding graduate award, Jilin University 
2013-2014 The first place student scholarship, Jilin University

\section{Publications}

1. Meng S, Whitt AG, Tu, Eaton JW, Li C, Yaddanapudi K (2019). Isolation of exosome-enriched extracellular vesicles carrying granulocytemacrophage colony-stimulating factor from embryonic stem cells. JoVE, manuscript accepted.

2. Yaddanapudi K, Meng S, Whitt AG, Al Rayyan N, Richie J, Tu A, et al. Exosomes from GM-CSF expressing embryonic stem cells are an effective prophylactic vaccine for cancer prevention. Oncoimmunology. 2019;8(3):1561119.

3. Neely AM, Zhao G, Schwarzer C, Stivers NS, Whitt AG, Meng S, et al. N(3-Oxo-acyl)-homoserine lactone induces apoptosis primarily through a mitochondrial pathway in fibroblasts. Cell Microbiol. 2018;20(1).

\section{Meeting Abstracts \& Poster Presentations}

1. 10/2014 18th Scientific Meeting of the Chinese Diabetes Society "The incidence of psychological change and its influence factors in patients with type 2 diabetes"

2. $06 / 2015$ 75th Scientific Sessions of American Diabetes Association "Association of AFF3 rs10865035 polymorphisms with the risk of Type 1 diabetes mellitus and autoimmune thyroid disease in a Chinese population" 
3. $07 / 2017$ Ohio Valley Chapter of the Society of Toxicology Summer Meeting

"Exosomes from embryonic stem cells function as prophylactic vaccines against lung cancer"

4. 10/2017 Research!Louisville

"Exosomes from embryonic stem cells function as prophylactic vaccines against lung cancer"

5. 10/2018 Research!Louisville

"Exosomes from embryonic stem cells against lung cancer as a prophylactic vaccine"

6. 04/ 2019 Experimental Biology Meeting

"A novel embryonic stem cell-based vaccine for the prevention against lung cancer"

7. 06/ 2019 Ohio Valley Chapter of the Society of Toxicology Summer Meeting

"A novel embryonic stem cell-based vaccine for the prevention against lung cancer"

8. 09/ 2019 Research!Louisville

"An embryonic stem cell-based vaccine in lung cancer prevention"

\section{Oral presentation}

1. 10/ 2014 18th Scientific Meeting of the Chinese Diabetes Society 
"The incidence of psychological change and its influence factors in patients with type 2 diabetes"

2. 11/2018 Department of Pharmacology and Toxicology, University of Louisville

"A novel embryonic stem cells-based vaccine for the prevention of lung cancer"

\section{Symposia Attended}

04/2019 IDeA National Resource for Proteomics Workshop, Little Rock, Arkansas 